\title{
Nonlinear Control of Plate Vibrations
}

\author{
Osama Naim Ashour
}

Dissertation submitted to the Faculty of the Virginia Polytechnic Institute and State University in partial fulfillment of the requirements for the degree of

Doctor of Philosophy

in

Engineering Mechanics

Ali H. Nayfeh, Chair

Dean T. Mook

Muhammad R. Hajj

Mehdi Ahmadian

Slimane Adjerid

January 17, 2001

Blacksburg, Virginia

Keywords: Active Control, Vibration Absorber, Saturation, Smart Materials, Piezoelectric Ceramics, Terfenol-D

Copyright 2001, Osama Naim Ashour 


\title{
Nonlinear Control of Plate Vibrations
}

\author{
Osama Naim Ashour
}

(ABSTRACT)

A nonlinear active vibration absorber to control the vibrations of plates is investigated. The absorber is based on the saturation phenomenon associated with dynamical systems with quadratic nonlinearities and a two-to-one internal resonance. The technique is implemented by coupling a second-order controller with the plate's response through a sensor and an actuator. Energy is exchanged between the primary structure and the controller and, near resonance, the plate's response saturates to a small value.

Numerical as well as experimental results are presented for a cantilever rectangular plate. For numerical studies, finite-element methods as well as modal analysis are implemented. The commercially available software ABAQUS is used in the finite-element analysis together with a user-provided subroutine to model the controller. For the experimental studies, the plate is excited using a dynamic shaker. Strain gages are used as sensors, while piezoelectric ceramic patches are used as actuators. The control technique is implemented using a dSPACE digital signal processing board and a modeling software (SIMULINK). Both numerical and experimental results show that the control strategy is very efficient.

A numerical study is conducted to optimize the location of the actuators on the structure to maximize its controllability. In this regard, the control gain is maximized for the PZT actuators. Furthermore, a more general method is introduced that is based on a global measure of controllability for linear systems.

Finally, the control strategy is made adaptive by incorporating an efficient frequency-measurement technique. This is validated by successfully testing the control strategy for a nonconventional problem, where nonlinear effects hinder the application of the non-adaptive controller. 


\section{Dedication}

To my mother Waela

To my daughter Waela 


\section{Acknowledgments}

I would like to express my sincere gratitude and appreciation to my advisor Dr. Ali H. Nayfeh. His invaluable knowledge and support were indispensable for this work. His encouragement, thoughtfulness, and supervision are deeply acknowledged. His ingeniousness, resourcefulness, and devotion are greatly admired.

Thanks are given to Dr. Dean Mook, Dr. Muhammad Hajj, Dr. Mehdi Ahmadian, and Dr. Slimane Adjerid for their contributions as committee members. Special thanks are due to Dr. Shafic Oueini, to whom I owe much of my knowledge in nonlinear control. His support and guidance were the hidden power behind this work. Thanks are also due to Dr. Sean Fahey. His experimental experience was of great help in this work. I would like to thank Dr. Jon Pratt, whose comments on Terfenol-D actuators were very helpful. I would like also to appreciate the help of Dr. Haider Arafat, who introduced me to the world of nonlinear dynamics.

I would like to express my deep gratitude and sincere appreciation to my parents. This work would not have come to existence without their patience, sacrifice, and insistence that I acquire a higher education. I am also deeply indebted to my wife and little daughter for their patience and support. I am forever grateful to my family for their love, kindness, and belief in me. 


\section{Contents}

1 Introduction $\quad 1$

1.1 Passive Vibration Absorbers . . . . . . . . . . . . . . . . 2

1.2 Adaptive Vibration Absorbers . . . . . . . . . . . . . . . 5

1.3 Active Vibration Absorbers . . . . . . . . . . . . . . . . 7

1.4 Nonlinear Vibration Absorbers . . . . . . . . . . . . . . . . . . . . 11

1.5 Autoparametric Vibration Absorbers . . . . . . . . . . . . . . 14

2 Analysis $\quad 25$

2.1 Modal Analysis . . . . . . . . . . . . . . . . . . 33

2.2 Perturbation Analysis . . . . . . . . . . . . . . . . 34

2.2.1 Equilibrium Solutions . . . . . . . . . . . . . . . 38

2.3 Finite-Element Method . . . . . . . . . . . . . . . . . . . 41

3 Numerical Results $\quad 46$

3.1 Finite-Element Method . . . . . . . . . . . . . . . . . . . . 47

3.2 Modal Analysis . . . . . . . . . . . . . . . . . . . 52

4 Optimal Placement of Actuators $\quad 57$

4.1 Optimal Placement By Maximizing Control Gain . . . . . . . . . . . . 58

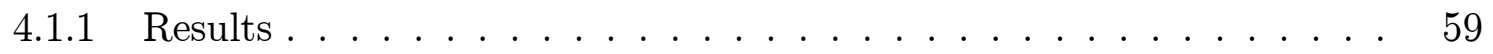

4.2 Optimal Placement Using a Measure of Modal Controllability . . . . . . . 63

4.2.1 State-Space Model . . . . . . . . . . . . . . 65 
$4.2 .2 \quad$ Results . . . . . . . . . . . . . . . . . . . . . . . . . . 67

5 Experimental Setup and Results $\quad 71$

5.1 Setup and Procedure . . . . . . . . . . . . . . . . . . . 72

5.2 Controller Circuit . . . . . . . . . . . . . . . . . . . . . . . . . . 74

5.3 Results . . . . . . . . . . . . . . . . . . . . . . 75

5.3 .1 Control of the First Mode . . . . . . . . . . . . . . . . 75

5.3.2 Control of the Second Mode . . . . . . . . . . . . . . . . . . . 81

5.3.3 Simultaneous Control of the First Two Modes . . . . . . . . . . . 87

6 Adaptive Control $\quad 92$

6.1 Frequency-Measurement Technique . . . . . . . . . . . . . . . . 93

6.2 Adaptive Control of a Beam with a Terfenol-D Actuator . . . . . . . . . . 94

$6.2 .1 \quad$ Experimental Setup . . . . . . . . . . . . . . . . . . . . 97

6.2 .2 Results . . . . . . . . . . . . . . . . . . . . . . . . . . . . . . . . . . 99

7 Concluding Remarks and Recommendations 104

7.1 Summary . . . . . . . . . . . . . . . . . . . . . . . . 104

7.1.1 Analytical Considerations . . . . . . . . . . . . . . 105

$7.1 .2 \quad$ Numerical Results . . . . . . . . . . . . . . . . . . . . . 106

7.1.3 Optimal Placement of Actuators . . . . . . . . . . . . . . . 106

7.1 .4 Experimental Work . . . . . . . . . . . . . . . . . . . . 107

7.1 .5 Adaptive Control . . . . . . . . . . . . . . . . . . . . . . . . 108

7.2 Recommendations for Future Work . . . . . . . . . . . . . . . 109

$\begin{array}{lr}\text { Bibliography } & 111\end{array}$

$\begin{array}{lr}\text { Vita } & 117\end{array}$ 


\section{List of Figures}

2.1 A schematic of a cantilever plate with a PZT actuator. . . . . . . . . . 26

3.1 The first six mode shapes of the cantilever plate. The 1,2, and 3 directions

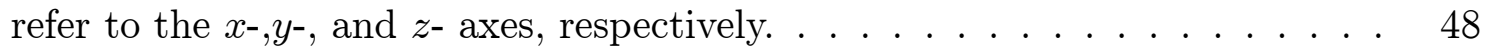

3.2 Effect of varying the feedback gain $\alpha$ : (a) $\alpha=0.005$, (b) $\alpha=0.015$, (c) $\alpha=0.06$, (d) $\alpha=0.075$, (e) $\alpha=0.085$, (f) $\alpha=0.09$, (g) $\alpha=0.11$, and (h) $\alpha=0.5 \ldots \ldots 50$

3.3 Effect of shear deformation and rotary inertia on the response of the plate: (a) this-shell element and (b) thick-shell element. . . . . . . . . . . . . 52

3.4 Plate displacement at the lower right corner using the first five modes. . . . 53

3.5 Plate displacement at the lower right corner using (a) the second through fifth modes and (b) the first mode only. . . . . . . . . . . . . . . 54

3.6 Frequency-response curves: (- - ) unstable and (-) stable response. . . . . 55

3.7 Frequency-response curves for quadratic velocity control: (- - ) unstable and $(-)$ stable response. . . . . . . . . . . . . . . . . . 56

4.1 Control gain variation over the domain of the plate for the first five modes: (a) mode 1 , (b) mode 2, (c) mode 3, (d) mode 4, and (e) mode 5. . . . . . 61

4.2 Optimum actuator location on the plate: (a) mode 1, (b) mode 2, (c) mode 3 , (d) mode 4 , and (e) mode $5 . \ldots \ldots \ldots$. . . . . . . . . . 62

4.3 Locations of modeling points on the plate for the discrete model. . . . . . . . 68

5.1 The experimental setup. . . . . . . . . . . . . . . . . . . 74

5.2 Control of the first mode of the plate . . . . . . . . . . 76

$5.3 \quad$ The plate response when $\alpha=0.1$ and $\gamma=1 \ldots \ldots \ldots \ldots$

5.4 Effect of varying the nonlinear feedback gain $\alpha$ : (a) $\alpha=0.01$, (b) $\alpha=0.05$,

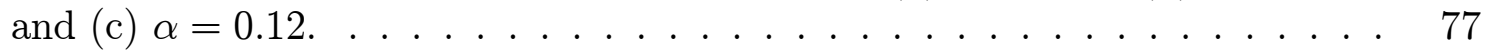


5.5 Effect of varying the nonlinear control gain $\gamma$ : (a) $\gamma=1.0$, (b) $\gamma=0.5$, and

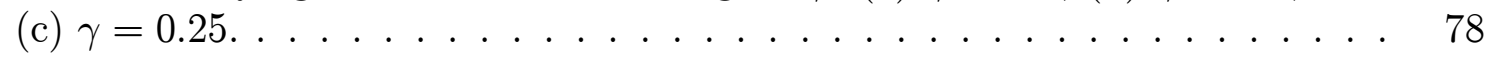

5.6 Effect of varying the initial controller displacement: (a) $v=0.5 \mathrm{~V}$, (b) $v=$ $1.0 \mathrm{~V}$, (c) $v=1.6 \mathrm{~V}$, and (d) $v=2.0 \mathrm{~V} \ldots \ldots \ldots \ldots$

5.7 Effect of varying the initial controller velocity: (a) $\dot{v}=0.5 \mathrm{~V} / \mathrm{sec}$ and (b)

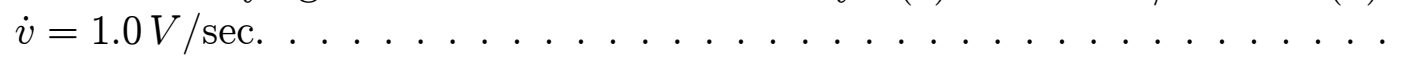

5.8 Effect of varying the controller damping coefficient $\xi$ : (a) $\xi=0.01$, (b) $\xi=$

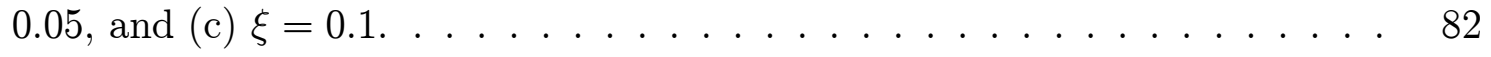

5.9 Frequency-response curves: (ooo) controller off and $(+++)$ controller on. . . 83

5.10 Force-response curves: (ooo) controller off and $(+++)$ controller on. . . . . 83

5.11 Plate response when $\alpha=0.075, \gamma=1.0$, and $F=11.9 \mathrm{mg}$ : $(+++)$ experimental results and (ooo) numerical results. . . . . . . . . . . 84

5.12 Control of the second mode of the plate: (a) plate response and (b) controller

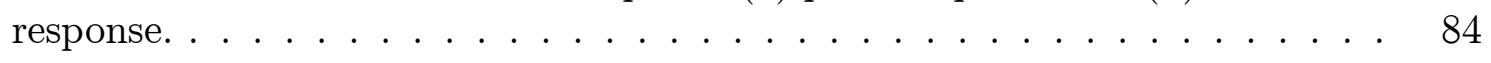

5.13 Effect of varying the feedback gain $\alpha$ : (a) $\alpha=0.04$, (b) $\alpha=0.07$, (c) $\alpha=0.1$, (d) $\alpha=0.11$, (e) $\alpha=0.12$, and (f) $\alpha=0.15 \ldots \ldots \ldots$

5.14 Effect of varying the initial displacement of the controller: (a) $v=0.03 \mathrm{~V}$, (b) $v=3.0 \mathrm{~V}$, and (c) $v=5.0 \mathrm{~V} \ldots \ldots \ldots \ldots \ldots$

5.15 Frequency-response curves for the second mode: (ooo) controller off and $(+++)$ controller on. . . . . . . . . . . . . . . .

5.16 Force-response curves for the second mode: (ooo) controller off and $(+++)$

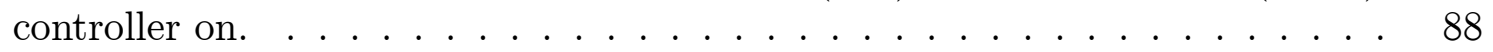

5.17 Simultaneous control of the first two modes of the plate: (a) plate response and $(\mathrm{b})$ response of the two controllers. . . . . . . . . . .

5.18 Effect of varying the feedback gain $\alpha$ : (a) $\alpha=0.01$, (b) $\alpha=0.02$, and (c)

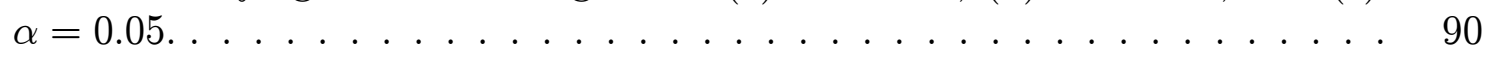

5.19 FFT results for the plate response: (a) no control and (b) with control. . . . 91

5.20 Power spectrum for the plate response with a noise signal: (a) no control and (b) with control. . . . . . . . . . . . . . . . . . . 91

6.1 Characteristics of a NAND Schmitt-trigger gate. . . . . . . . . . . 95

6.2 A typical assembly of a Terfenol-D actuator. . . . . . . . . . . . . 96

6.3 Displacement-current relationship for the used Terfenol-D actuator. . . . . . 97 
6.4 Experimental setup and control technique. . . . . . . . . . . . . . 99

6.5 Power spectrum of the output of the beam when excited at $f=13.31 \mathrm{~Hz}$. . 100

6.6 Control of the first mode of the beam when $f_{n}=11.875 \mathrm{~Hz}$ : (a) acceleration at the tip of the beam and (b) controller response. . . . . . . . . . . 101

6.7 FFT of the beam acceleration at the tip: (a) with control and (b) without

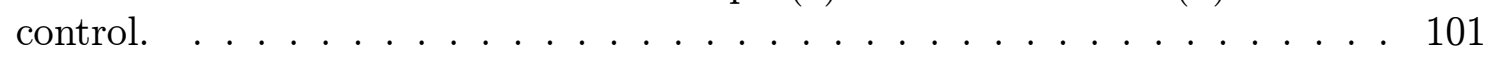

6.8 Nonadaptive control of the first mode of the beam: (a) acceleration at the tip of the beam and (b) controller response. . . . . . . . . . . . . 103 


\section{List of Tables}

4.1 Locations of maximum controllability . . . . . . . . . . . . . . 60

4.2 Locations of maximum controllability . . . . . . . . . . . . . . . . 69 


\section{Chapter 1}

\section{Introduction}

The dynamic response of mechanical and civil structures subject to high-amplitude motions is often dangerous and undesirable. The most detrimental form of these motions are oscillatory; thereby causing a vibrational motion. All mechanical systems are subjected to various conditions that may result in vibrational motion. These vibrations often lead to material fatigue, structural damage and failure, deterioration of system performance, and increased noise level. These effects are usually prominent around the natural frequencies of the system. Such vibrations cannot be tolerated and, therefore, developing a strategy for reducing these vibrations has been a major focus of research.

There are two fundamentally different primary methods of vibration protection. These are vibration isolation and vibration absorption. Other methods include modifying the vibrating system itself to accommodate for the new disturbance environment. However, this method is undesirable since internal system modifications are usually difficult to carry out. In vibration isolation, the original system is divided into two parts, which are connected by means of additional mechanical devices, such as springs and dampers. These devices are referred to 
as vibration isolators. Common examples include rubber mounts for machinery and shock absorbers for automobiles. Isolators are positioned between the source of disturbance and the object to be protected or, equivalently, between the object and its supporting base. Isolators reduce the magnitude of force transmitted from the vibrating object to its foundation or, equivalently, reduce the transmitted motion from the base to the object. Isolators filter unwanted vibrations above a particular frequency by properly choosing the isolator stiffness.

On the other hand, another system is attached to the original system in vibration absorption. Thus, another degree of freedom is added to the system. The parameters of the attached system are chosen so as to cause a decrease in the vibration level of the original system. These attached systems are referred to as vibration absorbers. Vibration absorbers reduce vibrations of the original system by channeling energy to the absorber itself. In classical vibration absorbers, this channeled energy is manifested as a deflection in the absorber's mass. While damping is essential for an isolator to reduce vibration levels at resonance, it defeats the purpose of an absorber. This is so because damping reduces the amount of energy transferred to the absorber.

\subsection{Passive Vibration Absorbers}

The concept of a vibration absorber was first outlined by Watts (1883), when addressing a method to reduce the rolling of ships. However, the first practical design of a vibration absorber was proposed by Frahm (1909). He designed a fluid tank system to reduce the rolling of ships. In his design, both the primary system and the absorber had no damping. 
The absorber was intended to be used at the fundamental resonant frequency of the main system. In this case, the absorber consisted of simply an auxiliary mass-spring system. Mathematically, it could be easily shown that when the absorber's natural frequency is tuned to the frequency of the main system, the resulting amplitude of vibration of the main system is reduced to zero. However, this design is only effective at or near the resonant frequency of the primary system. Attachment of the absorber introduces two resonant frequencies for the resulting two-degree-of-freedom system. These two frequencies are located on either side of the original resonant frequency. In reality, it can never be guaranteed that the frequency of operation will stay constant at the tuning frequency. For example, variation in the speed of rotating machinery is unavoidable. In addition, to reach the speed of operation, as well as to come to a complete stop, the system has to pass through the first natural frequency of the combined system. This will induce large transient vibration levels.

To remedy this problem, Ormondroyd and Den Hartog (1928) considered the case of a damped vibration absorber attached to the primary system. This resulted in a system effective over an extended frequency range by reducing the response at the two resonant frequencies of the combined system. However, the response at the primary system's natural frequency can no longer be reduced to zero. Hence, a trade off exists between the primary system's response and operating in a broadband. Later, Den Hartog (1956) and Snowdon (1968) described an optimization method for broadband applications to find the optimum tuning frequency and damping ratio. This method is known as the equal-peak method, which states that in the set frequency band, the two maximum values of amplitude on either 
side of the primary system's natural frequency are equal. Puksand (1975) applied the same criterion to the more general case of a varying force amplitude, such as a rotating unbalance. Soom and Lee (1983) examined other optimization criteria (objective functions) other than minimizing the maximum amplitude. Some of the examined criteria included minimizing the part of the frequency response where there is amplification of motion, minimizing the maximum velocity, and minimizing the mean-squared displacement response to noise excitation. A better broadband behavior was obtained, but at some cost in the allowable maximum amplitude. Semercigil, Lammers, and Ying (1992) suggested introducing an impact damper into the absorber to control the excessive vibrations of the primary system. Energy transferred to the absorber from the primary system is dissipated through impact between the mass of the absorber (secondary mass) and the impact damper. With this modification of the conventional tuned vibration absorber, significant attenuations were obtained in the displacement of the primary structure over a wide frequency range. However, there are two main shortcomings of this system. First, it is not effective when the primary system has significant inherent damping. Second, collisions between the impact damper and its boundaries produce large local stresses and, hence, have a catastrophic effect on the absorber itself.

The above mentioned vibration absorbers are passive devices. Vibrational energy is absorbed without the need for external energy. However, passive absorbers are effective only when the tuned frequency and damping are suitable for the particular operating conditions. Such conditions are often variables of time and, hence, passive vibration absorbers are often mistuned. This causes such absorbers to lose effectiveness and even amplify the vibration 
level instead of attenuating it. Alternatives to passive vibration control include adaptive (semi-active) and active control strategies.

\subsection{Adaptive Vibration Absorbers}

Adaptive vibration control involves incorporating a material with known controllable behavior into the system, and thus "tune" the response of the system to achieve a lower level of vibration. Thus, it becomes possible for the system (or structure) to adapt to a variable environment in the interest of continuously optimizing its performance. If, in addition, the system is modified to include sensory and feedback control capabilities, then the result is an intelligent material system. In general terms, such systems or structures are those that can sense external stimuli and react in an appropriate manner so as to optimally meet pre-specified performance criteria.

Adaptive vibration control was thought of as a less expensive alternative to fully active vibration control. Both energy and cost requirements are generally less. A recent review of adaptive tuned vibration absorbers was provided by Von Flotow, Beard, and Bailey (1994) where different issues related to the physical implementation of these absorbers were discussed. Tuning a passive vibration absorber to reduce vibration disturbances requires matching the absorber's natural frequency to that of the disturbance. Hollkamp and Starchville (1994) suggested a global search tuning algorithm. This method effectively scans the absorber's range of frequencies and automatically tunes the absorber's frequency to that which produces maximum reduction in the main system vibration level. However, this method is 
only valid for an invariant frequency of excitation. Francheck, Ryan, and Bernhard (1995) proposed and successfully implemented an adaptive vibration absorber based on a feedback tuning algorithm and a variable stiffness helical spring. The tuning algorithm is robust with respect to variation in the absorber parameters and the excitation frequency. The goal of the tuning algorithm is to minimize a DC signal, which is proportional to the main system's response, by changing the absorber's stiffness. Using a DC motor to rotate the helical spring, the effective number of coils can be changed and, hence, the absorber's stiffness is changed. A shortcoming of this approach is that the range of excitation frequencies must be known a priori in order to design the variable-stiffness spring. A maximum vibration attenuation of $24 \mathrm{~dB}$ was obtained in their experimental verification. Other variable-stiffness-spring designs are also available in the literature. Examples include an adjustable dual-leaf spring (Mianzo, 1992), a variable fulcrum positioning mechanism (Margolis and Baker, 1992), and a magnetic-field-based stiffness (Waterman, 1988).

Today, the biggest challenge to the use of this class of adaptive vibration absorbers is designing a practical and rugged adaptive absorber. Generally, several constraints, such as weight, size, and cost, complicate the design of such absorbers and make them application dependent. This may be the main reason that adaptive absorbers are not as widely used as their passive and active counterparts. Furthermore, as pointed out by Von Flotow, Beard, and Bailey (1994), the tuning range of the resonant frequency as well as the speed of tuning are limited by the too many involved electrical and mechanical components.

Another class of adaptive vibration absorbers includes the use of piezoelectric shunted cir- 
cuits. This provides a simpler, less expensive, and more practical approach than the modified passive absorbers discussed above. Numerous researchers suggested the use of such

absorbers, such as Hagood and Crawley (1989), Hagood and Von Flotow (1991), Browning and Wynn (1993), Hollkamp (1994), Agnes and Inman (1996), Davis, Lesieutre, and Dosch (1997), and Lesieutre (1998). The design of such absorbers consists of a pair of piezoelectric tiles attached to the system to be controlled and shunted by an inductor-resistor circuit. The resulting electrical resonance can be tuned to match the primary system's frequency, which results in vibration suppression. By this, the piezoelectric shunted circuit acts very similarly to the traditional mechanical mass-spring-damper vibration absorber. A simple control law can be used to tune the absorber, and hence make it adaptive by increasing or decreasing the electrical resonance.

\subsection{Active Vibration Absorbers}

A fully active control strategy involves using force actuators requiring external energy. The actuation force is used to improve the absorption level, and it is traditionally applied between the absorber's mass and the primary system parallel to the elastic (resilient) element that supports the absorber mass. This active absorber is then controlled with different algorithms, making it more sensitive to disturbances in the primary system. With the advance in modern control techniques, active vibration control is becoming more widely used than either the passive or the adaptive absorbers. In its simplest form, active control consists of sensors, electronic circuits, and actuators. The sensor feeds the electronic circuit (analog or digital) 
with a signal (feedback signal) which is proportional to the vibration level of the primary structure. The output of the electronic circuit is the actuation force law, which is fed into the actuators. The actuators, in turn, apply the opposing force on the primary structure. This control strategy allows for both better absorption characteristics and broadband applications.

Smart materials that undergo volumetric changes under certain external stimuli provide an excellent choice for sensors/actuators in active control strategies. The resulting vibration absorber can be of light weight, very compact, and reliable. Examples of such smart materials include piezoelectric (PZT) transducers, piezoelectric stacks, electrostrictive and magnetostrictive materials, and shape memory alloys. Other smart materials, such as electrorheologicl and magnetorheological fluids, which do not undergo volumetric changes, are mainly used in vibration isolation and adaptive vibration control.

There have been numerous studies on active vibration control. Noticeable contributions include the studies of Huang and Lian (1994), where an active dynamic absorber was designed using a DC servomotor as the actuator. A classical control strategy (PID) was employed to control the vibrations of a two-degree-of-freedom spring-lumped mass system. Reduction of the amplitude of vibration to one-fifth its original value was obtained. The results were shown to depend on the power of the used DC motor and the resolution of the A/D and D/A cards, which were used for I/O data processing. In addition, it was shown that the spring had a nonlinear behavior during compression deformation. Performance of this linear control strategy would not be optimal in this situation. Burdisso and Heilmann (1998) used two absorber's reaction masses attached to the primary structure through elastic elements. The 
active force was applied between these two masses, as opposed to the traditional configuration, where the force is applied between the absorber's mass and the primary structure. The actuating force was magnetic with the magnet fixed to one mass and the coil to the other mass. This force was controlled by a current passing through the coil. While this design resulted in the same control effort as that of the traditional passive absorber, it required much less power.

Recently, a novel approach called the delayed resonator was presented by Olgac and HolmHansen (1994). This concept combines a classical passive vibration absorber with a simple local linear feedback. The objective of this feedback is to convert the dissipative passive absorber into a conservative one with a certain resonance frequency. This results in placing two dominant poles of the characteristic equation on the imaginary axis of the complex root-locus plot. The feedback signal is proportional to the absorber's position, velocity, or acceleration, and utilizes a controlled time delay. The control strategy is to choose the proper feedback gain and time delay. By this, the absorber behaves like a resonator. In this case, the response of the primary structure can be brought to rest. This is a direct result of the fact that the absorber displacement is 180 degrees out-of-phase with the input disturbance. This active absorber does not need information from the primary structure. Hence, the absorber is autonomous and acts as a stand alone unit. Also, this absorber is tunable in real time, which results in effective absorption of disturbances with varying frequencies. Additionally, this absorber can be tuned to suppress multiple frequencies of vibrations. This is done by choosing the appropriate gain and time-delay parameters needed to set the corresponding 
pairs of poles on the imaginary axis. Thus, the single-mass delayed resonator can exhibit more than one natural frequency simultaneously. Because of the time delay, the characteristic equation of the absorber is transcendental, and thus has infinitely many roots. This fact causes a major concern regarding the stability of the combined system. This is so because the control parameters (gain and time delay) are chosen with no regard for the rest of the roots. Hence, a given passive absorber imposes some limitations on the delayed resonator. To ensure stability, Olgac and Holm-Hansen (1994) suggested that the operating absorber frequency must be greater than a critical frequency. Thus, there is a lower bound frequency limitation to this absorber. Olgac and Jalili (1998) implemented the delayed resonator on a flexible beam. Modal analysis was used to model the system together with unconventional boundary conditions. Qualitative agreement between the experimental and analytical findings was shown regarding the stability aspect of the combined system. A piezoelectric actuator was used to force the resonator at the designated frequency. Hosek, Olgac, and Elmali (1999) implemented the delayed resonator in a centrifugal vibration absorber to eliminate torsional oscillations in rotating mechanical structures.

Filipovic and Schroder (1999) used the same idea of the delayed resonator to design a linear active resonator. However, instead of the time delay, a linear compensator was used to convert the dissipative absorber into a resonator. Again, there can be as many roots on the imaginary axis as there are frequencies to be absorbed and, hence, the absorber can suppress multiple disturbance frequencies simultaneously. Several compensators were proposed, such as a proportional compensator with a single time constant, a proportional-integrative (PI), 
and a lead/lag compensator. Stability margins of the combined system were found by solving for the roots of the characteristic equation.

Fanson and Caughey (1990) were the first to present the positive position feedback technique. This technique makes use of a displacement feedback signal to suppress vibrations. Hence, the equation of motion of the main system is linearly coupled with the compensator's equation of motion. The compensator is composed of a second-order filter with a high damping ratio. The compensator's position measurement as well as the structure's displacement measurement are both positively fed to the filter and the structure, respectively. This technique has the advantage of being simple and straightforward. Also, stability of the system, although not unconditional, can be achieved in a way which is independent of the dynamical characteristics of the structure. The use of piezoelectric materials as sensors and actuators adds to the compactness and simplicity of this technique. However, this method is not efficient in dense modal environments.

\subsection{Nonlinear Vibration Absorbers}

All of the above research was based on linear control of linear systems. In reality, modeling of the dynamic behavior of vibrating systems must be generalized to include nonlinear effects. Any actual vibration absorber has restraints limiting the maximum allowed geometric boundaries of the linear region. Linear theory can be applied only for the cases in which the level of excitation results in deflections within the linear region. In contrast to the linear theory, the nonlinear behavior of a vibrating system depends on initial conditions. This 
means that the uniqueness of the steady-state response is no longer ensured. A linearly designed vibration absorber may not function properly in the nonlinear region. To avoid this problem, one increases the dissipative forces (damping) because damping is proven to reduce the nonlinear effects. However, this causes the performance of the absorber to deteriorate as was shown earlier. With the advances in technology and scientific research, the need to protect equipment used in aircraft, spacecraft, vessels, and other moving objects necessitates the inclusion of nonlinear effects, or at least finding a way to deal with them. To summarize, nonlinear effects exist in almost all vibration suppression systems and cannot be ignored, especially if the application of such systems limits the linear region. In addition, as will be shown later, the use of nonlinear control theory is proven to be superior over its linear counterpart. The recent application of the saturation phenomenon, which is encountered in nonlinearly coupled systems with a two-to-one internal resonance, proved that vibrations can be suppressed at a wide range of frequencies.

Nonlinear vibration absorbers can be divided into two main categories: absorbers with nonlinear elastic elements (springs) and nonlinear autoparametric absorbers. In this section, we discuss the first type; and in the next section, we discuss autoparametric absorbers.

The effectiveness of the nonlinear absorber was first outlined by Roberson (1952) who introduced the notion of a suppression bandwidth. This is basically the frequency range over which the ratio of the displacement amplitude to the amplitude of the forcing function is less than unity. In his analysis, he considered a linear plus cubic spring with no damping. He concluded that a softening spring would widen the suppression bandwidth. Arnold (1955) 
confirmed these results using a Ritz method instead of the iteration method used by Roberson. Hunt and Nissen (1982) suggested using a nonlinear softening spring of the Belleville type in a damped passive absorber. By this, it was shown that the suppression band can be widened; that is, the absorber could be used as a broadband one. This was mainly the case for low absorber-damping values. Rice and McCraith (1987) used a bow-type or shallow arch spring as the nonlinear coupling element. This spring was modeled as a Duffing-type spring with cubic nonlinearities and could be implemented as either a hardening or a softening type. They showed a relationship between the tuning frequency and a specific critical tuning frequency above which a hardening system was preferable and below which a softening type was advantageous. On the other hand, near this critical tuning frequency, the linear counterpart was more advantageous.

Harmonic instability would result if the system incorporated strong nonlinearities; quasiperiodic (multi-harmonic) and chaotic responses would result. Asymmetry in the spring was shown to have beneficial effects on the suppression bandwidth and stability of the system. Shaw, Shaw, and Haddow (1989) used perturbation methods (the method of multiple scales) to analyze the effect of nonlinearities in a nonlinear absorber with a Duffing-type spring. It was shown that a combination resonance (excitation frequency near the mean of the two linearized natural frequencies of the system) could occur. This results in large-amplitude quasi-periodic vibrations due to a Hopf bifurcation and, hence, eliminates the effectiveness of the absorber. Furthermore, the quasi-periodic solution coexists with the desired lowamplitude periodic solution. It was also shown that low damping, which is desirable in linear 
designs, promotes the onset of instability, leading to a modulated quasi-periodic response.

Gonsalves, Neilson, and Barr (1993) presented a design of a nonlinear absorber system in which the nonlinearity is a discontinuous stiffness provided by a clearance between the absorber mass and a linear spring. It was shown that this design facilitates a reduction in the amplitude of the second resonance peak. It also reduces the response of the auxiliary mass. However, the effectiveness of this absorber deteriorates as the level of damping increases. Also, quasi-periodic and chaotic responses of the primary mass occur just beyond the design frequency.

Lee and Shaw (1997) studied the dynamic response of a pair of identical centrifugal absorbers subject to external torques of multiple harmonics. The goal of the study was to develop an analytical formulation to predict the conditions at which the instability of the unison motion occurs. Using the method of multiple scales, they showed that this instability has a beneficial effect on the overall torsional vibration of the system. The pair of absorbers is thus capable of simultaneously canceling two harmonics when the damping level is small. Since the absorbers were unlocked in unison, their amplitudes and phases could be adjusted to cancel both the external excitation (primary) resonance of order $n$ and the induced superharmonic resonance of order $2 n$.

\subsection{Autoparametric Vibration Absorbers}

Parametric vibration is induced by a varying system parameter (stiffness). On the other hand, autoparametric vibration (self-parametric) is characterized by an internal coupling 
involving at least two modes. From a mathematical point, this coupling is effected by nonlinear terms in the equations of motion of the combined system . Physically speaking, an autoparametric system consists of two parts: a main system and a secondary system. The main system is externally excited, while the secondary system is parametrically excited as a result of the variation of its stiffness with the response of the main system. In other words, a two-mode interaction occurs when the main system exhibits a forced response which, in turn, drives the secondary system into parametric resonance. In this case, energy is transferred from one part of the combined system to the other. This energy transfer depends on the type of nonlinearities and the damping forces. It can be partial or complete, depending on the system parameters. In case of quadratic nonlinearities, the energy transfer is complete when the ratio of the main system's natural frequency to that of the secondary system is two to one. This case is referred to as a two-to-one internal resonance, which has been the focus of study for many researchers. For a more in-depth discussion of internal resonances in nonlinear systems, the reader is referred to Nayfeh and Mook (1979) and Nayfeh (2000).

In case of complete energy transfer from the main system to the secondary one, the main system's response can be suppressed. When the main system is excited at a frequency near its natural frequency (primary resonance), the main system's response will have the same exciting frequency. The response amplitude will linearly increase with the amplitude of excitation. However, after a critical value, the response saturates at a specific value and all additional energy from the excitor is channeled to the secondary system. This phenomenon is referred to as the saturation phenomenon. 
The saturation phenomenon was first discovered by Nayfeh, Mook, and Marshall (1973) while analyzing the coupling between the roll and pitch motions of ships. The saturation phenomenon was later experimentally demonstrated by Haddow, Barr, and Mook (1984). They investigated modal interactions in an L-shaped structure whose mathematical model included quadratic terms. By carefully tuning the natural frequencies, they were able to suppress the motion in the directly excited mode. Based on this, they suggested exploiting the saturation phenomenon as a vibration absorber. Oh, Nayfeh, and Mook (2000) studied experimentally and theoretically the loss of dynamic stability and the resulting large-amplitude roll of ships due to internal coupling. The excitation frequency was chosen to be twice the natural frequency in roll in the presence and absence of a two-to-one internal resonance between the pitch and roll modes. Force-response curves were obtained and the development of large-amplitude rolling motion was demonstrated. However, the heave and pitch waves reflecting from the sidewalls of the tank acted to limit the roll motion. Consequently, the saturation phenomenon was not experimentally observed.

Nayfeh and Zavodney (1988) further examined a physical structure similar to that of Haddow, Barr, and Mook (1984) consisting of two light-weight beams and two concentrated masses. The linear natural frequencies of the system were tuned so that they were approximately in the ratio of two to one and the excitation frequency was slowly varied around the lower natural frequency. They observed amplitude- and phase-modulated responses for a range of the excitation frequency. They identified regions of aperiodic motions (indicating the occurrence of a Hopf bifurcation), in qualitative agreement with the results of a second-order 
perturbation theory. Balachandran and Nayfeh (1991) extended this work and experimented with several flexible metallic and composite structures subjected to a range of resonant excitations. Again, the ratio of the system's natural frequencies was approximately two to one. Experimental observations of transitions from periodic to aperiodic and chaotic motions were observed even at low excitation levels.

While unaware of the saturation phenomenon, Haxton and Barr (1972) were among the first to investigate autoparametric vibration absorbers. Their model of an autoparametric absorber was based on a flexible spring-steel beam with a tip mass. Beam geometrical factors were shown to affect the absorber's behavior significantly. By adjusting the mass or the length so as to make the ratio of the natural frequency of the main system to that of the beam two to one, they were able to suppress vibrations of the main system. In their work, this type of absorber did not outperform the classical linear tuned vibration absorber. This was especially the case when large forcing levels were implemented. Ibrahim and Roberts (1976) studied the response of a two-degree-of-freedom system with autoparametric coupling to a broadband random excitation. They showed that close to the principal internal resonance (two-to-one), the secondary system underwent large random motions. This was accompanied by a suppression effect on the random motion of the main system. Also, it was shown that, under conditions of internal resonance, the random motions of both of the secondary and main systems were accompanied by steady harmonic responses.

Hatwal, Mallik, and Ghosh (1982) replaced the beam of Haxton and Barr by a rigid pendulum attached by a hinge joint to a block mass. In this case, the secondary system restoring force 
was either due to gravity or a torsional spring. Both cubic and quadratic nonlinearities were present in their model. The method of harmonic balance was used to show suppression of vibrations of the main system for both configurations. However, in a work published later (1983), they showed some interesting time responses for the pendulum with a chaotic behavior in both numerical and experimental results. Bajaj, Chang, and Johnson (1994) and Banerjee, Bajaj, and Davies (1993) used averaging methods to study the gravity-controlled pendulum of Hatwal, Mallik, and Ghosh (1982). Again, chaotic responses were reported in the response of the pendulum. In addition, the inclusion of higher-order nonlinearities in the model was shown to eliminate the saturation effect.

Cartmell and Roberts (1988) studied autoparametric interactions in a pair of harmonically excited coupled beams with three and four mode resonances. In this case, two simultaneous combination resonances in the secondary system were activated by the response of the primary system. They also showed that a third combination resonance could be inferred when the other two were close to each other. Ashworth and Barr (1987) considered four-mode interactions in a model for an aircraft T-tail structure with quadratic inertial nonlinearities. The model was tested under direct and parametric harmonic excitations. Nayfeh, Nayfeh, and Mook (1994) theoretically and experimentally examined a T-shaped structure consisting of two light-weight beams and three concentrated masses at the upper and lower free ends and at the point were the beams were joined. The lengths and weights were chosen so that the third natural frequency was approximately equal to the sum of the two lower natural frequencies. Periodic, two-period quasiperiodic, and phase-locked (synchronized) motions 
were observed. In addition, the saturation phenomenon was experimentally demonstrated in the case of combination internal resonance.

Cartmell and Lawson (1994) gave specifics of a preliminary design of an autoparametric vibration absorber with a bolt-on inverted pendulum-type beam. The design was semi-active in the sense that the tuning frequency was varied according to the main system's response, which was measured by an accelerometer. As in the pendulum of Hatwal, Mallik, and Ghosh (1982), the directly excited primary mode drove the pendulum into principal parametric resonance. The method of multiple scales was used to analytically find an approximate solution to the equations of motion. Experimentally, two helical springs were used to restrain the pendulum-type beam to its vertical equilibrium position. The control strategy consisted of adjusting the position of the lumped mass according to a computer signal. The detuning was either continued or reversed according to the primary system response. The optimal point would be achieved when the response amplitude was a minimum. This was shown to broaden the suppression region. The main disadvantage of this design is the high cost associated with the needed accuracy and delicacy to adjust the lumped mass position by the drive motor.

Golnaraghi (1991) proposed using a sliding mass-spring-dashpot mechanism placed at the free end of a cantilever beam. This resulted in nonlinear coupling through Coriolis, inertia, and centripetal accelerations between the slider and the beam. Numerical simulations were conducted to control the transient vibrations of the free response of the beam. For small values of controller damping, chaotic transient oscillations were observed for large initial 
disturbances of the beam. Duquette, Tuer, and Golnaraghi (1993) experimentally used a DC motor, a secondary beam, and a tip mass to control the transient vibrations of a cantilever beam.

Cuvlaci and Ertas (1996) studied the dynamic response of a beam-tip mass-pendulum system subject to harmonic excitations. The nonlinear equations of motion were developed and then reduced, using a Galerkin expansion, to two coupled equations for the first two modes. Nonlinear terms appear due to the curvature of the beam and the coupling between the beam and the pendulum. Both numerical and experimental results showed significant reduction in the main system (beam) response at the primary resonance condition (two-to-one internal resonance) since complete energy transfer between the two modes occurred at that frequency. Also, their results revealed that energy exchange from the beam to the pendulum was significantly increased, as well as a larger suppression region was obtained, when the forcing level was increased. However, it was found that for smaller pendulum masses, the response experiences quasi-periodic and chaotic motions.

Yabuno, Endo, and Aoshima (1999) proposed a stabilization method for subharmonic resonance of order one-third using an autoparametric absorber. The subharmonic resonance was created by a nonlinear (cubic) spring stiffness and harmonic excitation. The controller was a damped pendulum system whose natural frequency was about one-half that of the main system. This induced the two-to-one internal resonance and the subharmonic resonance of order one-third was suppressed. Furthermore, it was theoretically proven that, in the presence of the absorber, there was no stable nontrivial steady-state responses with respect to 
the one-third subharmonic component. The only stable solution was the trivial one. Their experimental results verified the proposed method.

All of the above nonlinear autoparametric vibration absorbers suffer from two main problems. First, there is an added complexity and design difficulty in coupling two mechanical systems together. Second, it is almost impossible to maintain the two-to-one internal-resonance condition by such controllers. Also, the used mechanical controller (pendulum, beam) actually induces higher-order nonlinearities and not just quadratic. When either the forcing amplitude is large or the controller's frequency is far away from the internal-resonance condition, these other nonlinear terms counteract the suppression efficiency. As a result, undesired high-amplitude quasi-periodic and chaotic motions evolve.

Oueini and Golnaraghi (1996) proposed using a linear second-order analog electronic-circuit controller coupled to the vibrating structure via quadratic nonlinear terms. These nonlinear terms acted as a bridge for energy exchange between the structure and the controller. This strategy was implemented to suppress the transient free vibrations of a DC motor equipped with position feedback. Subsequently, Khajepour and Golnaraghi (1997) implemented this technique on a cantilever beam actuated by PZT patches. However, a digital electronic circuit was used via a digital signal processing (DSP) board and a PC. The method of normal forms was used to derive the nonlinear control law to maximize energy transfer between the plant and the controller. Both free- and forced-vibration cases were considered. Pai et al. (1998) used the saturation phenomenon and internal-resonance conditions to control the transient and steady-state vibrations of a cantilever beam using PZT patches as 
actuators and sensors. A digital control system was used as the controller. Both numerical and experimental results showed time histories of the plant's response with and without control. No frequency- or force-response curves were shown to illustrate the effectiveness and robustness of the technique. In addition, their numerical results were valid only around resonance, and no data was given regarding the system behavior away from resonance. Realtime system vibrations include such detuned cases and, therefore, any control method must account for them. Furthermore, the approach did not allow for automatic tuning of the absorber frequency.

Oueini, Nayfeh, and Pratt (1998) and Pratt, Oueini, and Nayfeh (1999) developed an active nonlinear vibration absorber, which exploited the saturation phenomenon. A linear secondorder analog electronic circuit was used as a controller, which was coupled to the plant through a sensor and an actuator. Both the feedback and control signals were quadratic. As actuators, they used PZT patches and Terfenol-D. Terfenol-D is a magnetostrictive material that exhibits a nonlinear constitutive relation between the magnetic field and the strain. This nonlinear relationship was taken to be quadratic to the first approximation. In this case, saturation could be achieved by merely tuning the controller's frequency to one-half that of the plant. Their experiments demonstrated the effectiveness of this technique in controlling the first and second modes of vibration of a cantilever beam. However, the Terfenol-D experiments were not as effective as the PZT experiments. This was attributed to the detuning between the controller's frequency and that of the plant. Oueini and Nayfeh (2000) used a digital signal processing (DSP) board instead of the analog circuit. Hence, the 
controller's system parameters, such as the natural frequency, damping, and feedback gains, could be adjusted digitally. Furthermore, automatic tracking of the plant's frequency was made possible.

In this work, we extend the method of Oueini and Nayfeh (2000) and study the vibrations of two-dimensional systems. The saturation phenomenon has thus far been used in onedimensional systems (beams). In this work, we intend to investigate and demonstrate the effectiveness of using the saturation phenomenon to control the vibrations of two-dimensional systems, such as plates. In addition, the saturation phenomenon is implemented to control both bending and torsional vibration modes. As a case study, we use a cantilever rectangular plate. Both a finite-element method and a discretization technique based on modal analysis are used to model the problem. In the finite-element method, a nonlinear model incorporating nonlinear geometrical factors is used. Hence, nonlinear effects in the primary system are included in the analysis. The finite-element package (ABAQUS) is also modified to allow for feedback control using internal resonance and the saturation phenomenon. Thus, the control method can be numerically simulated even if the eigenfunctions of the system cannot be obtained analytically. Experimental studies are also conducted on a rectangular cantilever plate. Strain gages are used as sensors, whereas PZT patches are used as actuators. PZT patches have the advantage of being light and small in volume. Hence, they are perfect for aerospace and other structures where added volume and mass constitute a constraint on the application. The experimental part also aims to develop an adaptive control strategy using an effective frequency-tuning mechanism, such that the two-to-one internal-resonance 
condition is maintained. In nonlinear problems, the frequency of the system changes with the amplitude. So, a continuous tuning mechanism is needed to keep the controller's frequency tuned as the amplitude of the vibrating structure changes. This will demonstrate the robustness of the control method. Furthermore, we study the problem of optimizing the location of the actuators on the plant to maximize its controllability. In this regard, two methods are introduced. The first method is based on maximizing the magnitude of the controller gain factor, which is obtained from analytical considerations. The second method is based on a global measure of controllability for linear systems.

In Chapter 2, we start by modeling the plate vibrations with the PZT actuator. The governing equations of motion are derived. Modal analysis is then used to solve for the plate's response using its natural modes. A finite-element method utilizing the software ABAQUS is used to calculate the natural modes as well as to solve for the plate's response. A brief description of the software is also presented. In Chapter 3, we show the numerical results and simulations. In Chapter 4, we discuss the problem of optimizing the actuators locations on the plant. The experimental setup and results are discussed in Chapter 5, while a discussion of the frequency-measurement technique and its applications is presented in Chapter 6. Finally, discussion of the results as well as the conclusions and recommendations for future work are presented in Chapter 7. 


\section{Chapter 2}

\section{Analysis}

In this chapter, we analyze the problem of a vibrating cantilever plate with a piezoelectric (PZT) actuator bonded to its surface. We first derive the equations of motion with the appropriate boundary conditions, and then use modal analysis to solve for the plate's response represented by the lateral displacement $w$.

We consider a thin plate with length $a$, width $b$, and thickness $h$. The plate is assumed to have a uniform mass density $\rho$ per unit area, elastic modulus $E$, Poisson's ratio $\nu$, and a viscous damping coefficient $C$ per unit area. The plate is harmonically excited by a shaker with an amplitude $F$. The edge $x=0$ is fixed as shown in Fig. 1 . The PZT actuators are bonded to the top and bottom surfaces of the plate and extend from $x=x_{1}$ to $x=x_{2}$ and from $y=y_{1}$ to $y=y_{2}$, as shown in Fig. 1. The PZT actuators have a thickness $t_{a}$, modulus of elasticity $E_{p z}$, Poisson's ratio $\nu_{p z}$, and a piezoelectric constant $d_{31}$.

For a piezoelectric element, when a voltage $V_{a}$ is applied along its polarization direction $z$, strains are induced in both the $x-$ and $y$ - directions. These strains can cause extension or 


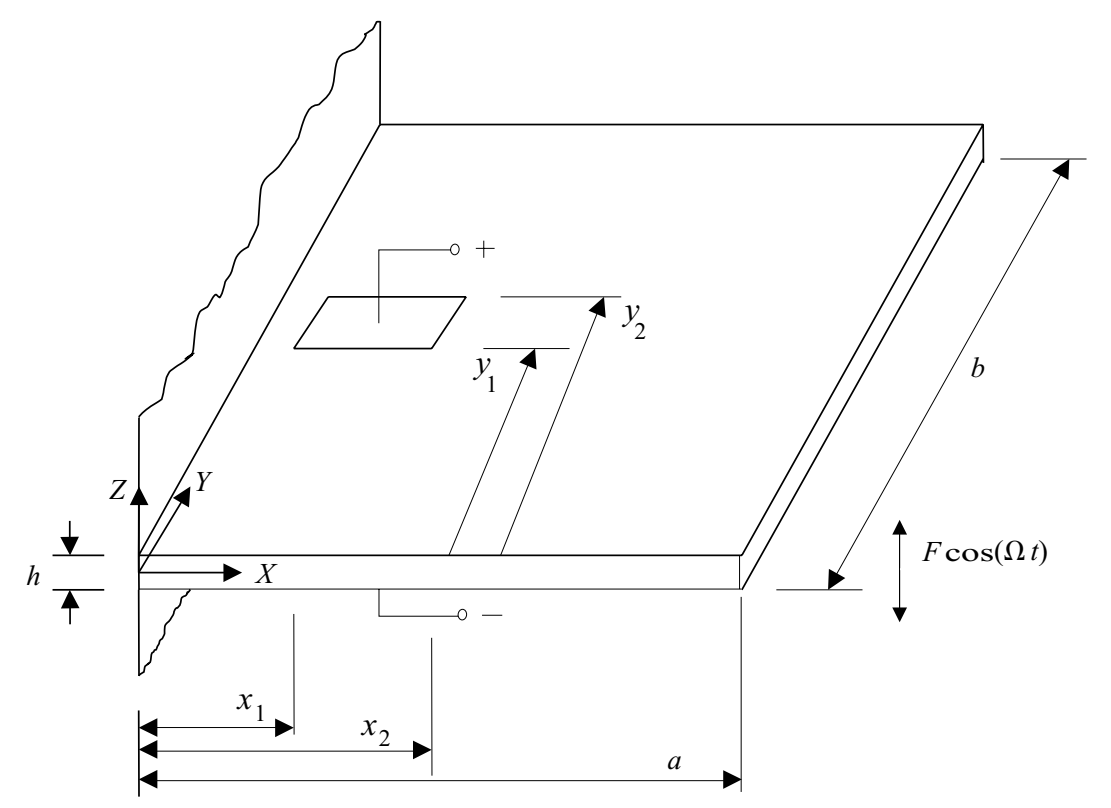

Figure 2.1: A schematic of a cantilever plate with a PZT actuator.

contraction, depending on the sign of the applied voltage. Both of the induced strains in the $x$ - and $y$ - directions are equal, and for an unconstrained (free) piezoelectric element, their magnitude is given by

$$
\epsilon_{p z}=\frac{d_{31}}{t_{a}} V_{a}
$$

Thus, when a PZT pair is bonded on the top and bottom surfaces of the plate and the corresponding signs of the applied voltages are opposite, the PZT patches will strain in opposite directions. This causes a net bending moment on the plate. A case of pure bending will arise if the PZT pair is identical and bonded in symmetry with respect to the plate's neutral axis. Hence, no net extension or contraction of the plate exists.

Crawley and de Luis (1987) studied the effect of the bonding layer on the transmitted strains and stresses, which cause the resulting bending moments. They concluded that, as the shear 
modulus of the bonding layer increases or the thickness of this layer decreases, the shear is effectively transferred over a small area close to the end of the PZT patch. For the case of perfect bonding, such as the case considered here, the strain is entirely transferred between the piezoelectric and the plate over an infinitesimal area near the ends of the PZT actuator. Hence, the shear force is transferred at a concentrated point at the edge of the PZT. The resulting bending moments are uniformly distributed along the boundary of the PZT. Thus, the PZT pair can be replaced by uniformly distributed edge moments applied to the plate at the interface between the PZT and the plate surface.

To derive the equation of motion of the plate, we use the extended Hamilton principle (Meirovitch, 1997) given by

$$
\int_{t_{1}}^{t_{2}}\left(\delta T-\delta V+\delta W_{n c}\right) d t=0 \quad \delta w=0 \text { at } t=t_{1}, t_{2}
$$

Here, $\delta$ is the variation operator, $T$ is the kinetic energy, $V$ is the strain (potential) energy, and $W_{n c}$ is the work due to the non-conservative forces. The kinetic energy is given as the integral of the differential kinetic energy over the area of the plate as

$$
T=\int_{0}^{b} \int_{0}^{a} \frac{1}{2} \rho \dot{w}^{2} d x d y
$$

The integral of the variation in the kinetic energy is found as follows:

$$
\begin{aligned}
\int_{t_{1}}^{t_{2}} \delta T & =\int_{0}^{b} \int_{0}^{a} \int_{t_{1}}^{t_{2}} \rho \dot{w} \frac{\partial}{\partial t} \delta w d t d x d y \\
& =\int_{0}^{b} \int_{0}^{a}\left\{(\rho \dot{w} \delta w)_{t_{1}}^{t_{2}}-\int_{t_{1}}^{t_{2}} \frac{\partial}{\partial t}(\rho \dot{w}) \delta w d t\right\} d x d y \\
& =-\int_{t_{1}}^{t_{2}} \int_{0}^{b} \int_{0}^{a} \rho \ddot{w} \delta w d x d y d t
\end{aligned}
$$


The non-conservative forces are the viscous damping forces, which are proportional to the velocity $\dot{w}$ and act opposite to the velocity direction. The variation of the work due to these forces is

$$
\delta W_{n c}=-\int_{0}^{b} \int_{0}^{a} 2 \mu \dot{w} \delta w d x d y
$$

The strain energy is due to the internal and external bending moments and the internal twisting moment. To proceed, we follow Timoshenko and Woinowsky-Krieger (1959) and express the strain energy due to the internal bending and twisting moments as

$$
V=-\int_{0}^{b} \int_{0}^{a} \frac{1}{2}\left(M_{x i} \frac{\partial^{2} w}{\partial x^{2}}+M_{y i} \frac{\partial^{2} w}{\partial y^{2}}-M_{x y} \frac{\partial^{2} w}{\partial x \partial y}\right) d x d y
$$

where $M_{x i}$ and $M_{y i}$ are the internal bending moments per unit length acting on the edges parallel to the $y$ - and $x$ - axes, respectively. These moments are related to the curvature of the plate by the following relationships:

$$
\begin{aligned}
& M_{x i}=-D\left(\frac{\partial^{2} w}{\partial x^{2}}+\nu \frac{\partial^{2} w}{\partial y^{2}}\right) \\
& M_{y i}=-D\left(\frac{\partial^{2} w}{\partial y^{2}}+\nu \frac{\partial^{2} w}{\partial x^{2}}\right)
\end{aligned}
$$

where $D$ is the flexural rigidity of the plate given by $\frac{E h^{3}}{12\left(1-\nu^{2}\right)}$. In addition to these bending moments, a twisting moment $M_{x y}$ exists when the $x$ - and $y$ - axes do not coincide with the principal planes of curvature. The twisting moment per unit length is given by

$$
M_{x y}=D(1-\nu) \frac{\partial^{2} w}{\partial x \partial y}
$$


The variation of the strain energy due to these internal bending and twisting moments is given as (Timoshenko and Woinowsky-Krieger, 1959)

$$
\begin{aligned}
\delta V= & D \int_{0}^{b} \int_{0}^{a}\left(\frac{\partial^{4} w}{\partial x^{4}}+2 \frac{\partial^{4} w}{\partial x^{2} \partial y^{2}}+\frac{\partial^{4} w}{\partial y^{4}}\right) \delta w d x d y \\
& -\oint M_{n} \frac{\partial \delta w}{\partial n} d s-\oint\left(\frac{\partial}{\partial s} M_{n t}-Q_{n}\right) \delta w d s
\end{aligned}
$$

where $M_{n}$ is the bending moment at a point on the boundary of the plate and $\frac{\partial}{\partial s} M_{n t}-Q_{n}$ is the effective shear force acting along the boundary of the plate. These are given by

$$
\begin{aligned}
M_{n}=- & D(1-\nu)\left[\frac{\partial^{2} w}{\partial x^{2}} \cos ^{2} \theta+2 \frac{\partial^{2} w}{\partial x \partial y} \sin \theta \cos \theta+\frac{\partial^{2} w}{\partial y^{2}} \sin ^{2} \theta\right]+\nu\left[\frac{\partial^{2} w}{\partial x^{2}}+\frac{\partial^{2} w}{\partial y^{2}}\right] \\
Q_{e f f}= & Q_{n}-\frac{\partial}{\partial s} M_{n t} \\
= & -D\left[\left(\frac{\partial^{3} w}{\partial x^{3}}+\frac{\partial^{3}}{\partial x \partial y^{2}}\right) \cos \theta+\left(\frac{\partial^{3} w}{\partial y^{3}}+\frac{\partial^{3}}{\partial y \partial x^{2}}\right) \sin \theta\right] \\
& +D(1-\nu) \frac{\partial}{\partial s}\left[\left(\frac{\partial^{2} w}{\partial x^{2}}-\frac{\partial^{2} w}{\partial y^{2}}\right) \sin \theta \cos \theta-\frac{\partial^{2} w}{\partial x \partial y}\left(\cos ^{2} \theta-\sin ^{2} \theta\right)\right]
\end{aligned}
$$

where $\theta$ is the angle between the outer normal and the $x$-axis. For a rectilinear edge parallel to the $y$-axis, $\theta=0$, and for a rectilinear edge parallel to the $x$-axis, $\theta=\pi / 2$. Expressing the surface integral in terms of moments, we rewrite equation (2.12) as

$$
\begin{aligned}
\delta V= & \int_{0}^{b} \int_{0}^{a}\left(\frac{\partial^{2} M_{x i}}{\partial x^{2}}-2 \frac{\partial^{2} M_{x y}}{\partial x \partial y}+\frac{\partial^{2} M_{y i}}{\partial y^{2}}\right) \delta w d x d y \\
& -\oint M_{n} \frac{\partial \delta w}{\partial n} d s-\oint\left(\frac{\partial}{\partial s} M_{n t}-Q_{n}\right) \delta w d s
\end{aligned}
$$

In exactly a similar manner, we can now account for the external bending moments $M_{x}$ and $M_{y}$ caused by the actuator noting that, however, they act opposite to the internal moments 
$M_{x i}$ and $M_{y i}$. Hence, the variation in the strain energy finally becomes

$$
\begin{aligned}
\delta V= & \int_{0}^{b} \int_{0}^{a}\left\{D\left(\frac{\partial^{4} w}{\partial x^{4}}+2 \frac{\partial^{4} w}{\partial x^{2} \partial y^{2}}+\frac{\partial^{4} w}{\partial y^{4}}\right)-\frac{\partial^{2} M_{x}}{\partial x^{2}}-\frac{\partial^{2} M_{y}}{\partial y^{2}}\right\} \delta w d x d y \\
& -\oint M_{n} \frac{\partial \delta w}{\partial n} d s-\oint\left(\frac{\partial}{\partial s} M_{n t}-Q_{n}\right) \delta w d s
\end{aligned}
$$

Now, substituting for $\delta T, \delta V$, and $\delta W_{n c}$ in equation (2.2) yields

$$
\begin{gathered}
\int_{t_{1}}^{t_{2}}\left\{\int_{0}^{b} \int_{0}^{a}\left\{-\rho \ddot{w}-2 \mu \dot{w}-D\left(\frac{\partial^{4} w}{\partial x^{4}}+2 \frac{\partial^{4} w}{\partial x^{2} \partial y^{2}}+\frac{\partial^{4} w}{\partial y^{4}}\right)+\frac{\partial^{2} M_{x}}{\partial x^{2}}+\frac{\partial^{2} M_{y}}{\partial y^{2}}\right\} \delta w d x d y\right. \\
\left.+\oint M_{n} \frac{\partial \delta w}{\partial n} d s+\oint\left(\frac{\partial}{\partial s} M_{n t}-Q_{n}\right) \delta w d s\right\} d t=0
\end{gathered}
$$

which, for an arbitrary $\delta w$, can only be satisfied if

$$
-\rho \ddot{w}-2 \mu \dot{w}-D \nabla^{4} w+\frac{\partial^{2} M_{x}}{\partial x^{2}}+\frac{\partial^{2} M_{y}}{\partial y^{2}}=0
$$

where

$$
\nabla^{4} w=\frac{\partial^{4} w}{\partial x^{4}}+2 \frac{\partial^{4} w}{\partial x^{2} \partial y^{2}}+\frac{\partial^{4} w}{\partial y^{4}}
$$

The line integrals give the boundary conditions as

$$
\begin{array}{cccc}
M_{n}=0 & \text { or } & \frac{\partial w}{\partial n}=0 \\
& \text { and } & \\
Q_{\text {eff }}=0 & \text { or } & w=0
\end{array}
$$

For the fixed edge $x=0$, the boundary conditions are $\frac{\partial w}{\partial n}=0$ and $w=0$. For the other three free edges, $M_{n}=0$ and $Q_{e f f}=0$.

For the case of base excitation using a shaker, such as the case considered here, the total lateral displacement $w_{t}$ is the sum of the base displacement $w_{b}$ and the plate displacement $w$ 
relative to the base. If the input shaker's displacement is harmonic and given by $-F \cos (\Omega t)$, then the equation of motion of the plate becomes

$$
\rho \ddot{w}+2 \mu \dot{w}+D \nabla^{4} w=\frac{\partial^{2} M_{x}}{\partial x^{2}}+\frac{\partial^{2} M_{y}}{\partial y^{2}}+\rho \Omega^{2} F \cos (\Omega t)
$$

To complete the derivation of the equation of motion, the external bending moments $M_{x}$ and $M_{y}$ need to be determined. To this end, we first note that, for the case of perfect bonding, the strain is continuous at the interface between the actuator and the plate. However, since the elastic modulus for the actuator is different from that of the plate, the stress is discontinuous. Also, the piezoelectric strain in the $x$ - direction is equal to that in the $y$ - direction, and hence the corresponding moments in the $x$ - and $y$ - directions are equal. Using thin plate theory, which assumes linear strain distributions through the plate, we can write Hooke's law for the stresses $\sigma_{x}$ and $\sigma_{y}$ in the plate at the interface with the actuator (i.e., at $z=h / 2$ ) as

$$
\begin{aligned}
\sigma_{x i} & =\frac{-E h}{2\left(1-\nu^{2}\right)}\left(\frac{\partial^{2} w}{\partial x^{2}}+\nu \frac{\partial^{2} w}{\partial y^{2}}\right) \\
\sigma_{y i} & =\frac{-E h}{2\left(1-\nu^{2}\right)}\left(\frac{\partial^{2} w}{\partial y^{2}}+\nu \frac{\partial^{2} w}{\partial x^{2}}\right)
\end{aligned}
$$

If we substitute for the bending moments $M_{x}$ and $M_{y}$, we obtain the following relationship between the moments and the stresses at the interface:

$$
M_{x}=M_{y}=\frac{1}{6} \sigma_{x i} h^{2}
$$

To find $\sigma_{x i}$, we use Hooke's law together with the assumption of linear stress distributions in both the plate and the actuator. Then, from the equilibrium of moments about the midplane of the plate, we have

$$
\int_{-h / 2}^{h / 2} \sigma_{x} z d z=-\int_{h / 2}^{h / 2+t_{a}}\left\{\left(\sigma_{x i}\right)_{p z}-\sigma_{x i}\left(1-\frac{z}{h}\right)\right\} z d z
$$


Hence, we obtain the following relationship between the plate strains at the interface and the unconstrained actuator strain:

$$
\epsilon_{x i}=\epsilon_{y i}=C_{0} \epsilon_{p z}
$$

where $C_{0}$ is given by

$$
C_{0}=\frac{6 E_{p z}\left(1+\nu_{p z}\right)\left(1-\nu^{2}\right) t_{a} h\left(h+t_{a}\right)}{E(1+\nu)\left(1-\nu_{p z}^{2}\right)\left(h^{3}+8 t_{a}^{3}+6 h t_{a}^{2}\right)+6 E_{p z}\left(1+\nu_{p z}\right)\left(1-\nu^{2}\right) t_{a} h\left(h+t_{a}\right)}
$$

Now, $\sigma_{x i}$ is easily found and substituted back into equation (2.24) together with the unconstrained piezoelectric strains given by equation (2.1) to solve for the bending moments $M_{x}$ and $M_{y}$. The result is

$$
M_{x}=M_{y}=C_{1} V_{a}
$$

where $C_{1}$ is given by

$$
C_{1}=\frac{C_{0} E h^{2} d_{31}}{6(1-\nu) t_{a}}
$$

Having determined the magnitudes of the external bending moments induced by the actuator, we now account for the fact that the actuator extends from $x=x_{1}$ to $x_{2}$ and $y=y_{1}$ to $y_{2}$ by using the unit step function $H$ and obtain

$$
M_{x}=M_{y}=C_{1} V_{a}\left[H\left(x-x_{1}\right)-H\left(x-x_{2}\right)\right]\left[H\left(y-y_{1}\right)-H\left(y-y_{2}\right)\right]
$$

Finally, substituting for $M_{x}$ and $M_{y}$ in equation (2.22), we obtain the final form for the equation of motion as

$$
\begin{aligned}
\rho \ddot{w}+2 \mu \dot{w}+D \nabla^{4} w= & \rho \Omega^{2} F \cos (\Omega t)+\left\{C_{1}\left[\delta_{1}^{\prime}\left(x-x_{1}\right)-\delta_{1}^{\prime}\left(x-x_{2}\right)\right]\left[H\left(y-y_{1}\right)-H\left(y-y_{2}\right)\right]\right. \\
& \left.+C_{1}\left[\delta_{1}^{\prime}\left(y-y_{1}\right)-\delta_{1}^{\prime}\left(y-y_{2}\right)\right]\left[H\left(x-x_{1}\right)-H\left(x-x_{2}\right)\right]\right\} V_{a}
\end{aligned}
$$


Here, $\delta_{1}$ is the Dirac delta function and the prime indicates the derivative with respect to the argument.

\subsection{Modal Analysis}

We expand the plate lateral deflection $w(x, y, t)$ as

$$
w(x, y, t)=\sum_{n=1}^{\infty} \phi_{n}(x, y) q_{n}(t)
$$

where the $\phi_{n}(x, y)$ are the linear undamped mode shapes and the $q_{n}(t)$ are the generalized coordinates. The mode shapes can be obtained using any natural frequency extraction numerical method (Meirovitch, 1980). In this study, we use the finite-element package ABAQUS. The mode shapes are orthonormalized such that

$$
\int_{0}^{b} \int_{0}^{a} \rho \phi_{r} \phi_{s} d x d y=\delta_{r s}
$$

where $\delta_{r s}$ is the Kronecker delta. Substituting back in the equation of motion, we obtain

$$
\begin{aligned}
& \sum_{n=1}^{\infty}\left(\rho \phi_{n}(x, y) \ddot{q}_{n}(t)+2 \mu \phi_{n}(x, y) \dot{q}_{n}(t)+D\left(\nabla^{4} \phi_{n}(x, y)\right) q_{n}\right)=\rho \Omega^{2} F \cos (\Omega t) \\
& \quad+\left\{C_{1}\left[\delta_{1}^{\prime}\left(x-x_{1}\right)-\delta_{1}^{\prime}\left(x-x_{2}\right)\right]\left[H\left(y-y_{1}\right)-H\left(y-y_{2}\right)\right]\right. \\
& \left.\quad+C_{1}\left[\delta_{1}^{\prime}\left(y-y_{1}\right)-\delta_{1}^{\prime}\left(y-y_{2}\right)\right]\left[H\left(x-x_{1}\right)-H\left(x-x_{2}\right)\right]\right\} V_{a}
\end{aligned}
$$

Multiplying equation $(2.34)$ by $\phi_{m}(x, y)$ and integrating the result over the domain of the plate yields

$$
\ddot{q}_{n}+\hat{\mu} \dot{q}_{n}+\omega_{n}^{2} q_{n}=P_{m} \cos (\Omega t)+\gamma V_{a}
$$

where $P_{m}=\rho \Omega^{2} F \int_{0}^{b} \int_{0}^{a} \phi_{m}(x, y) d x d y, \hat{\mu}=2 \mu / \rho$, and $\gamma$ is given by 


$$
\gamma=C_{1}\left\{\int_{y_{1}}^{y_{2}}\left(\frac{\partial \phi_{m}}{\partial x}\left(x_{2}\right)-\frac{\partial \phi_{m}}{\partial x}\left(x_{1}\right)\right) d y+\int_{x_{1}}^{x_{2}}\left(\frac{\partial \phi_{m}}{\partial y}\left(y_{2}\right)-\frac{\partial \phi_{m}}{\partial y}\left(y_{1}\right)\right) d x\right\}
$$

The input voltage is chosen to be quadratic to utilize the saturation control phenomenon as follows:

$$
V_{a}=K_{1} v^{2}
$$

where $K_{1}$ is a constant gain and $v$ is governed by

$$
\ddot{v}+2 \xi \dot{v}+\lambda^{2} v=\alpha w v
$$

Here, $\xi$ is the damping coefficient and $\lambda$ is chosen such that $2 \lambda \approx \Omega$.

\subsection{Perturbation Analysis}

We perform a perturbation analysis to derive an analytical solution to the controlled response. In this regard, the method of multiple scales (Nayfeh, 1981) is applied directly to the partial-differential equation (2.31) and the controller's equation (2.38). To this end, we seek a uniform approximation to $w$ (the plate lateral displacement) and $v$ (the controller response) in the form

$$
\begin{aligned}
& w \approx \epsilon w_{1}\left(x, y, T_{0}, T_{1}\right)+\epsilon^{2} w_{2}\left(x, y, T_{0}, T_{1}\right) \\
& v \approx \epsilon v_{1}\left(T_{0}, T_{1}\right)+\epsilon^{2} v_{2}\left(T_{0}, T_{1}\right)
\end{aligned}
$$

where $T_{0}=t$ is a fast-time scale and $T_{1}=\epsilon t$ is a slow-time scale describing the time evolution of the amplitudes and phases of the response. The derivatives with respect to time 
are expressed in terms of the new time scales as

$$
\begin{gathered}
\frac{\partial}{\partial t}=D_{0}+\epsilon D_{1}+\cdots \\
\frac{\partial^{2}}{\partial t^{2}}=D_{0}^{2}+2 \epsilon D_{0} D_{1}+\cdots
\end{gathered}
$$

where $D_{k} \equiv \frac{\partial}{\partial T_{k}}$. We first scale the damping coefficients and the forcing term as

$$
\mu \rightarrow \epsilon \mu \quad \xi \rightarrow \epsilon \xi \quad F \rightarrow \epsilon^{2} F
$$

Substituting in the equations of motion and equating coefficients of equal powers of $\epsilon$ leads to two sets of problems of order $\epsilon$ and $\epsilon^{2}$ :

Order $\epsilon$ :

$$
\begin{array}{r}
D_{0}^{2} w_{0}+\frac{1}{\rho} D \nabla^{4} w_{0}=0 \\
D_{0}^{2} v_{0}+\lambda^{2} v_{0}=0
\end{array}
$$

Order $\epsilon^{2}$ :

$$
\begin{aligned}
D_{0}^{2} w_{1}+\frac{1}{\rho} D \nabla^{4} w_{1}= & -\frac{2 \mu}{\rho} D_{0} w_{0}-\frac{2}{\rho} D_{0} D_{1} w_{0}+\Omega^{2} F \cos \left(\Omega T_{0}\right) \\
& +\frac{1}{\rho}\left\{C_{1}\left[\delta_{1}^{\prime}\left(x-x_{1}\right)-\delta_{1}^{\prime}\left(x-x_{2}\right)\right]\left[H\left(y-y_{1}\right)-H\left(y-y_{2}\right)\right]\right. \\
& \left.+C_{1}\left[\delta_{1}^{\prime}\left(y-y_{1}\right)-\delta_{1}^{\prime}\left(y-y_{2}\right)\right]\left[H\left(x-x_{1}\right)-H\left(x-x_{2}\right)\right]\right\} v_{0}^{2} \\
D_{0}^{2} v_{1}+\lambda^{2} v_{1}= & -2 D_{0} D_{1} v_{0}-2 \xi D_{0} v_{0}+\alpha w_{0} v_{0}
\end{aligned}
$$

The general solutions of equations (2.42) and (2.43) are

$$
\begin{aligned}
w_{0}\left(x, y, T_{0}, T_{1}\right) & =\sum_{n=1}^{\infty} \phi_{n}(x, y) A_{n}\left(T_{1}\right) e^{i \omega_{n} T_{0}}+c c \\
v_{0}\left(T_{0}, T_{1}\right) & =B_{1}\left(T_{1}\right) e^{i \lambda T_{0}}+c c
\end{aligned}
$$


where $c c$ stands for the complex conjugate of the preceding terms.

From a physical perspective, it can be argued that, for a damped system, the modes that are not directly excited by an external source or indirectly excited by an internal resonance decay with time. Hence, denoting the excited mode by the subscript $n$, we can write the solution for $w_{0}$ as

$$
w_{0}=\phi_{n} A_{n}\left(T_{1}\right) e^{i \omega_{n} T_{0}}+c c
$$

Substituting for $w_{0}$ and $v_{0}$ in equations (2.44) and (2.45), we obtain

$$
\begin{aligned}
D_{0}^{2} w_{1}+\frac{1}{\rho} D \nabla^{4} w_{1}= & -\frac{2 i \omega_{n}}{\rho} \phi_{n}\left[\mu A_{n}+A_{n}^{\prime}\right] e^{i \omega_{n} T_{0}}+\frac{1}{2} \Omega^{2} F e^{i \Omega T_{0}} \\
& +\frac{1}{\rho}\left\{C_{1}\left[\delta_{1}^{\prime}\left(x-x_{1}\right)-\delta_{1}^{\prime}\left(x-x_{2}\right)\right]\left[H\left(y-y_{1}\right)-H\left(y-y_{2}\right)\right]\right. \\
& \left.+C_{1}\left[\delta_{1}^{\prime}\left(y-y_{1}\right)-\delta_{1}^{\prime}\left(y-y_{2}\right)\right]\left[H\left(x-x_{1}\right)-H\left(x-x_{2}\right)\right]\right\} \\
& \times\left(B_{1}^{2} e^{2 i \lambda T_{0}}+B_{1} \bar{B}_{1}\right)+c c \\
D_{0}^{2} v_{1}+\lambda^{2} v_{1}= & -2 i \lambda\left[\xi B_{1}+B_{1}^{\prime}\right] e^{i \lambda T_{0}}+\alpha \phi_{n} A_{n}\left[B_{1} e^{i\left(\omega_{n}+\lambda\right) T_{0}}+\bar{B}_{1} e^{i\left(\omega_{n}-\lambda\right) T_{0}}\right]+c c
\end{aligned}
$$

To express the nearness of the excitation frequency to the natural frequency of the plate (primary resonance condition), we introduce $\sigma_{2}$ as a detuning parameter. On the other hand, the nearness of the plate's natural frequency to twice that of the controller (two-toone internal resonance condition) is expressed by the detuning parameter $\sigma_{1}$. Thus,

$$
\Omega=\omega_{n}+\epsilon \sigma_{2} \quad \text { and } \quad \omega_{n}=2 \lambda+\epsilon \sigma_{1}
$$

Because the associated homogeneous equations of equations (2.49) and (2.50) have nontrivial solutions, the nonhomogeneous equations have solutions only if solvability conditions are sat- 
isfied. These solvability conditions demand that the nonhomogeneous terms of each equation are orthogonal to every solution of the adjoint. However, since the system is self-adjoint, the orthogonality condition demands that the right-hand side of equation (2.49) be orthogonal to $\phi_{n}(x, y) e^{-i \omega_{n} T_{0}}$. Using the normalization condition (2.33), we obtain

$$
2 i\left[\mu A_{n}+A_{n}^{\prime}\right]-f e^{i \sigma_{2} T_{1}}-4 \hat{\gamma} B_{1}^{2} e^{-i \sigma_{1} T_{1}}=0
$$

where

$$
f=\frac{\Omega^{2} \rho^{2} F}{2 \omega_{n}} \int_{0}^{b} \int_{0}^{a} \phi_{n}(x, y) d x d y
$$

and

$$
\hat{\gamma}=\frac{\rho}{4 \omega_{n}} C_{1}\left\{\int_{y_{1}}^{y_{2}}\left(\frac{\partial \phi_{n}}{\partial x}\left(x_{2}\right)-\frac{\partial \phi_{n}}{\partial x}\left(x_{1}\right)\right) d y+\int_{x_{1}}^{x_{2}}\left(\frac{\partial \phi_{n}}{\partial y}\left(y_{2}\right)-\frac{\partial \phi_{n}}{\partial y}\left(y_{1}\right)\right) d x\right\}
$$

Similarly, the solvability condition for equation (2.50) demands the vanishing of the terms that produce secular terms. Hence, the solvability condition for this equation is

$$
2 i\left[\xi B_{1}+B_{1}^{\prime}\right]-4 \hat{\alpha} A_{n} \bar{B}_{1} e^{i \sigma_{1} T_{1}}=0
$$

where $\hat{\alpha}=\frac{\alpha}{4 \lambda} \phi_{n}$.

Using polar representations of $A_{n}$ and $B_{1}$ in the form

$$
A_{n}=\frac{1}{2} a_{n}\left(T_{1}\right) e^{i \beta_{n}\left(T_{1}\right)} \quad \text { and } \quad B_{1}=\frac{1}{2} b_{1}\left(T_{1}\right) e^{i \beta_{1}\left(T_{1}\right)}
$$

and separating the real and imaginary parts in equations (2.52) and (2.55), we obtain the following equations describing the modulation of the amplitudes and phases of the plate and 
controller response:

$$
\begin{aligned}
a_{n}^{\prime} & =-\mu a_{n}-\hat{\gamma} b_{1}^{2} \sin \gamma_{1}+f \sin \gamma_{2} \\
b_{1}^{\prime} & =-\xi b_{1}-\hat{\alpha} a_{n} b_{1} \sin \gamma_{1} \\
a_{n} \beta_{n}^{\prime} & =-\hat{\gamma} b_{1}^{2} \cos \gamma_{1}-f \cos \gamma_{2} \\
b_{1} \beta_{1}^{\prime} & =-\hat{\alpha} a_{n} b_{1} \cos \gamma_{1}
\end{aligned}
$$

where

$$
\gamma_{1}=\sigma_{1} T_{1}-2 \beta_{1}+\beta_{n} \quad \text { and } \quad \gamma_{2}=\sigma_{2} T_{1}-\beta_{n}
$$

\subsubsection{Equilibrium Solutions}

The fixed points or equilibrium solutions of the modulation equations (2.57) are obtained by setting $a_{n}^{\prime}, b_{1}^{\prime}, \gamma_{1}^{\prime}$, and $\gamma_{2}^{\prime}$ equal to zero and solving the resulting algebraic equations. In this case, there are two possible solutions depending on whether $b_{1}$ is equal to zero or not.

Case one: $b_{1}=0$. Solving for $a_{n}$ yields

$$
a_{n}=\frac{f}{\sqrt{\sigma_{2}^{2}+\mu^{2}}}
$$

which is essentially the linear solution for the problem when no nonlinear coupling is present. In this case, the amplitude of the plate response is directly proportional to the excitation amplitude. 
Case two: $b_{1} \neq 0$. In this case, the following solution is obtained:

$$
\begin{aligned}
& a_{n}=\frac{\sqrt{\xi^{2}+\frac{1}{4}\left(\sigma_{1}+\sigma_{2}\right)^{2}}}{|\hat{\alpha}|} \\
& b_{1}=\sqrt{\frac{\Lambda_{1} \pm\left(\hat{\alpha}^{2} f^{2}-\Lambda_{2}^{2}\right)^{\frac{1}{2}}}{\hat{\alpha} \hat{\gamma}}}
\end{aligned}
$$

where

$$
\Lambda_{1}=\frac{1}{2} \sigma_{2}\left(\sigma_{1}+\sigma_{2}\right)-\xi \mu \quad \text { and } \quad \Lambda_{2}=\frac{1}{2} \mu\left(\sigma_{1}+\sigma_{2}\right)+\xi \sigma_{2}
$$

Since $\sigma_{1}+\sigma_{2}=\frac{\Omega-2 \lambda}{\epsilon}$, we obtain the following expressions for the amplitudes for the case of perfect tuning between the excitation frequency and twice the controller frequency:

$$
\begin{aligned}
& a_{n}=\frac{\xi}{|\hat{\alpha}|} \\
& b_{1}=\sqrt{\frac{-\xi \mu \pm\left(\hat{\alpha}^{2} f^{2}-\xi^{2} \sigma_{2}^{2}\right)^{\frac{1}{2}}}{\hat{\alpha} \hat{\gamma}}}
\end{aligned}
$$

Interestingly, we note that the amplitude of the plate is not a function of the excitation amplitude as in the linear solution. This constitutes the basis of the control strategy; by minimizing the controller damping and adjusting its frequency to one-half the exciting frequency, the plate response can be effectively suppressed.

The stability of the equilibrium solutions of the system of equations (2.57) was studied by many researchers. For example, Nayfeh (2000) defined a critical value for the forcing $f$ by

$$
f_{c}=\sqrt{\frac{\xi^{2}\left(\sigma_{2}^{2}+\mu^{2}\right)}{\hat{\alpha}^{2}}}
$$

The system of equations (2.57) was then reformulated in Cartesian coordinates. The stability of a given fixed point to a disturbance proportional to $e^{s T_{1}}$ was determined by checking the 
roots of the characteristic equation of the eigenvalue problem $\dot{x}=A x$, where $x$ is the state vector. They found out that for $f \leq f_{c}$, only the linear solution exists. This solution is a sink. When $f \geq f_{c}$, this fixed point becomes a saddle, with one eigenvalue being real and positive.

The stability of the nonlinear solution was investigated by substituting for $a_{n}$ and $b_{1}$ from equations (2.59) and (2.60) into the characteristic equation and checking the resulting eigenvalues. The two conditions for local stability (around resonance) were found to be (Nayfeh, 2000)

$$
\hat{\alpha} \hat{\gamma} b_{1}^{2}+\xi \mu-\frac{1}{2} \sigma_{2}\left(\sigma_{2}+\sigma_{1}\right) \geq 0
$$

and

$$
4 \xi \mu\left(\mu^{2}+\sigma_{2}^{2}\right)\left(4 \xi^{2}+4 \mu \xi+\mu^{2}+\sigma_{2}^{2}\right)+8(\mu+\xi)^{2}\left(\mu^{2}+2 \mu \xi+\sigma_{2}\left(\sigma_{2}+\sigma_{1}\right)+\sigma_{2}^{2}\right) \hat{\alpha} \hat{\gamma} b_{1}^{2} \geq 0
$$

The violation of condition (2.63) indicates the existence of an eigenvalue with a positive real part. In this case, a saddle-node bifurcation occurs. On the other hand, the violation of condition (2.64) indicates the existence of a pair of complex eigenvalues with positive real parts. A Hopf-bifurcation is said to occur in this case. However, these conditions are automatically satisfied when the exciting frequency is perfectly tuned with twice the controller frequency, since $\sigma_{1}+\sigma_{2}=\frac{\Omega-2 \lambda}{\epsilon}$. Hence, the nonlinear solution is stable. This solution can be either a stable focus or a stable node, indicating either an oscillatory or a smoothly-decaying transient response, respectively. Again, this behavior is determined by examining the roots of the characteristic equation. Oueini, Nayfeh, and Pratt (1998) found 
out that below a critical value of $\hat{\alpha}$, all of the roots are real, indicating a smoothly-decaying response. This is the case when $\sigma_{2}=0$. When $\sigma_{2} \neq 0$, the transient response is always oscillatory, regardless of the value of $\hat{\alpha}$.

\subsection{Finite-Element Method}

For the finite-element analysis, we use the commercially available software ABAQUS. In the analysis, we used linear elastic behavior and plane-stress shell elements to model the plate. To include transverse shear-deformation and rotary-inertia effects, we used an orthotropic material behavior. The values of Young's modulus, Poisson's ratio, and shear moduli associated with the material's principal directions are needed. On the other hand, for thin-shell behavior, the transverse shear and rotary inertia are neglected and an isotropic material behavior is assumed. In this case, only Young's modulus and Poisson's ratio are needed.

Moderately thick plates are roughly measured by the ratio of the least lateral dimension to the plate thickness being under 20. Alternatively, this is measured by the plate thickness being more than $1 / 15$ of a characteristic length on the surface of the plate, such as the wavelength of a significant natural mode in dynamic analysis. In ABAQUS, the element type S8R is used to model thick shell problems. This element is eight noded and quadrilateral that uses a reduced integration scheme to form the element stiffness matrix. The mass matrix and distributed loadings are still integrated exactly. Reduced integration provides accurate results at a significantly less running time. This element approximates the Mindlin-

type element, which accounts for first-order shear deformations through the thickness and 
rotary-inertia effects. Hence, this element is suitable for the analysis of composite and sandwich plates and shells. The Mindlin model of plates weakens the Kirchhoff hypothesis by removing the assumption that material lines that are normal to the plate remain normal to the deformed middle surface. However, the assumption that these normals remain straight and unstrained after deformation is retained. In other words, the essential idea of this element is that the position of a point in the plate reference surface and that in the corresponding normal vector are interpolated independently. In ABAQUS, the transverse shear stiffness is computed by matching the shear response for the case of the shell bending about one axis. A parabolic variation of the transverse shear stress in each layer of the shell section is used. In other words, the transverse shear-stiffness coefficients are estimated by matching the elastic strain energy associated with the shear deformation of the shell section with that based on a piecewise quadratic variation of the transverse shear stress across the section under conditions of bending about one axis. For thin shells, these coefficients are scaled down by a factor that converges to zero as the thickness tends to zero.

For homogeneous plates, thin plates are characterized by their thicknesses being less than $1 / 15$ of a characteristic length on the surface, such as the wavelength of a significant mode. The element type S9R5 is used in ABAQUS to model thin shell/plate problems. This element is nine noded and quadrilateral that uses a reduced integration scheme similar to that used for thick shell problems. This element accurately satisfies the classical Kirchhoff plate theory, which states that material lines that are originally normal to the middle surface remain straight and normal to the deformed middle surface. Also, these lines undergo neither 
contraction nor extension. Hence, transverse shear effects are neglected. In small displacement theory, this assumption leads to strain-displacement relations that are accurate up to terms of $O\left(h^{2}\right)$. In ABAQUS, the Kirchhoff constraint can either be imposed analytically or numerically. When the constraint is imposed analytically, the solution involves no transverse shear-strain-energy calculations. However, when the constraint is imposed numerically, such as in the case of element S9R5, the transverse shear stiffness acts as a penalty to enforce the constraint. In this regard, the actual transverse shear stiffness of the section is multiplied by a factor to prevent the shear stiffness from becoming too large in thin shells. This factor converges to zero as the thickness of the plate tends to zero. For thin shell elements, the number of degrees of freedom at the element nodes can be reduced to five: three displacement components and two in-surface rotation components. This makes such elements more economical than the rest. However, they are available only for small-strain applications, although they model large in-surface rotations accurately.

In addition to thin and thick shell elements, ABAQUS provides finite-strain elements for a large-deformation analysis. Large-amplitude excitation of plates is one example of such finitestrain cases. These elements provide robust and accurate solutions. They allow for uniform change in the plate section's thickness. This analysis requires the inclusion of geometric nonlinearities. In this case, the strain-displacement relationship is nonlinear. When the transverse deflection of a plate is not small compared to its thickness, stretching of the middle surface occurs, which results in nonlinear strain-displacement relationships. The stress-strain relationships are, however, still linear. If these nonlinearities are ignored, the elements are 
formulated in the original reference configuration using the original nodal coordinates. The errors in this case are of the same order of magnitude as the involved strains and rotations. Nonlinear phenomena, such as postbuckling bifurcation analysis, cannot be captured using a geometrically linear analysis. On the other hand, when nonlinear geometries are included, the elements are formulated in the current configuration using current nodal coordinates. This means that the elements can distort from their original shapes as the deformation increases. For very large deformations, the elements may no longer be suitable for analysis. In ABAQUS, the element type S4R is used for finite-strain analysis.

In this study, the plate is divided into $40 \times 20$ shell elements. Both element types S9R5 and S8R are used to check for the tranverse-shear and rotary-inertia effects. Nonlinear geometry effects are accounted for by using element type S4R and by setting the NLGEOM option on. To model the actuators, we attach two piezoelectric thin plates to the top and bottom surfaces of the plate. Hence, the piezoelectric actuators respond by straining to the electrical potential gradient resulting from the output of the controller's response. The direction of this straining for the top actuator is made opposite to that of the bottom one by reversing the sign of the electrical potential gradient. Hence, a moment is generated on the plate. The piezoelectric properties are specified as either stress or strain material coefficients. The values of the piezoelectric constants $e_{i j k}$, defining the electrical flux in the direction $i$ caused by the $j k$ component of strain, correspond to those used in real experiments. A tied contact is assumed to exist between the plate (master surface) and the piezoelectric plates (slave surfaces). This means that the translational degrees of freedom of both contacting surfaces 
are constrained to move together throughout the analysis. Each node on the slave surface is constrained with the closest point on the master surface where the normal to the master surface passes through the node on the slave surface. Hence, there is no relative motion between the surfaces. In real life, this case corresponds to that of a perfect bond between the actuator and the plate surface. On the other hand, relative motion between the surfaces can be accounted for by including the small sliding option. If nonlinear geometries are ignored, then the sliding becomes infinitesimal.

To model the controller in ABAQUS, we choose a second-order structure that simulates a mass-spring-damper system. The chosen structure was a cantilever beam with the lowest natural frequency being equal to one-half that of the plate's mode to be controlled. This resulted in the two-to-one internal resonance and the saturation phenomenon. The natural frequency of the beam (controller) was chosen by selecting the appropriate modulus of elasticity, density, and geometric dimensions. Coupling between the controller (beam) and the plate was introduced by adding a subroutine to the ABAQUS input file, which allowed for reading the response of the plate (displacement or strain) and that of the controller. The product of the output of the plate, the beam response, and the feedback gain is then fed to the original code as a force on the controller (beam). Also, the output of the controller is squared and multiplied by the control gain. The result is fed to the original code as a voltage on the PZT actuator. This simulated a real-life feedback control experiment where only physical elements are used. 


\section{Chapter 3}

\section{Numerical Results}

In general, real-life structures are complex. Furthermore, it is not always easy and inexpensive to build a prototype for these structures. Hence, numerically simulating the controlled behavior of such structures becomes extremely important before actually implementing the control strategy. Numerical results give us a good estimate of the real-life response, especially when we take into account all of the possible variables affecting the response. For our control strategy, we need first to estimate the natural frequencies and the mode shapes of the plate. Then, we need to locate the nodal lines for each mode, so as to avoid placing the actuator on these lines. A numerical study in Chapter 4 is conducted to locate the optimal location of the actuator for maximum control efficiency. Furthermore, for our control strategy, we need to estimate the nonlinear gains to be used in the experiments and get a general idea about the trend of the response. The numerical results will also verify the analytical

findings, which we discussed in Chapter 2. Unlike the perturbation solution, the numerical results also allow us to solve for the response away from resonance and check the global stability of the system. 
We present numerical results for the controlled and uncontrolled responses of the vibrating plate. Two numerical methods are discussed here. In the first method, we model the cantilever plate, the PZT actuator, and the controller using the finite-element package ABAQUS, which fully integrates the mathematical model over time. In the second method, we use ABAQUS to calculate the natural modes (eigenfunctions) and frequencies (eigenvalues) of the cantilever plate. These modes are then used in a modal analysis to solve for the plate response (displacement).

\subsection{Finite-Element Method}

We perform numerical simulations for an aluminum plate with dimensions $9.00^{\prime \prime} \times 7.50^{\prime \prime} \times$ $0.03^{\prime \prime}$. The plate is divided into $40 \times 20$ shell elements. Both thin and thick shell elements are used to check for transverse-shear and rotary-inertia effects. The natural frequencies of the cantilever plate are extracted using the FREQUENCY subroutine. The first six obtained natural frequencies are: $17.9,37.9,98.7,116.8,145.4$, and $233.2 \mathrm{~Hz}$. These values agree very well with the experimental results, where an FRF analysis is used together with a fine-tuning procedure to locate the frequency with the maximum amplitude. The first six mode shapes are shown in Fig. 3.1.

Next, we obtain the time-history response for different values of the feedback gain $\alpha$, namely, $\alpha=0.005,0.015,0.06,0.075,0.085,0.09,0.11$, and 0.5 . The forcing (base excitation) is set at 11.9 mg. The results are shown in Fig 3.2, where we plot the strain (in microns) at a distance $x=0.18^{\prime \prime}$ and $y=2.16^{\prime \prime}$ from the lower left corner. In Fig. 3.2(a), the gain was so small 

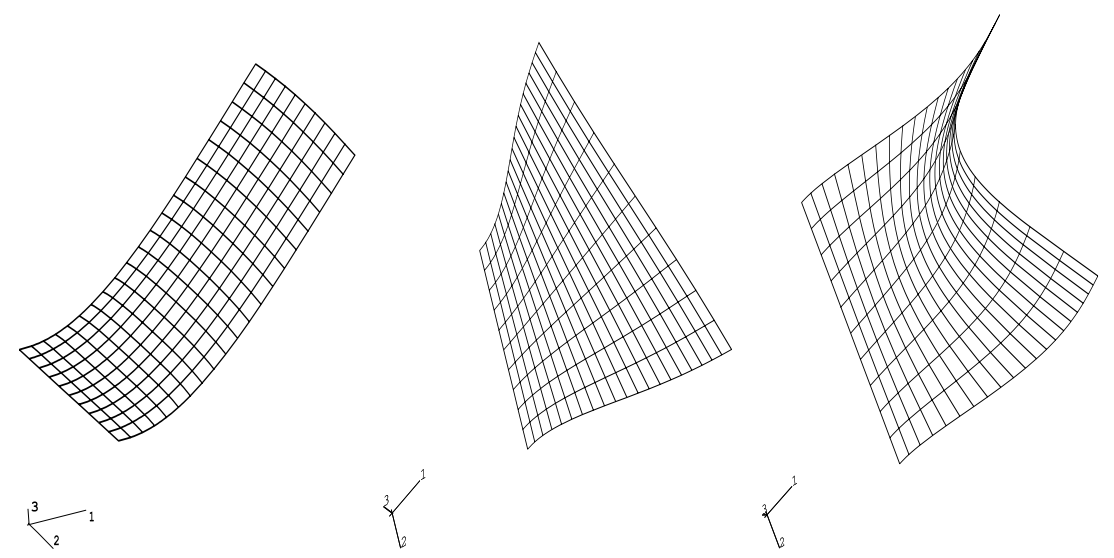

(a)

(b)

(c)
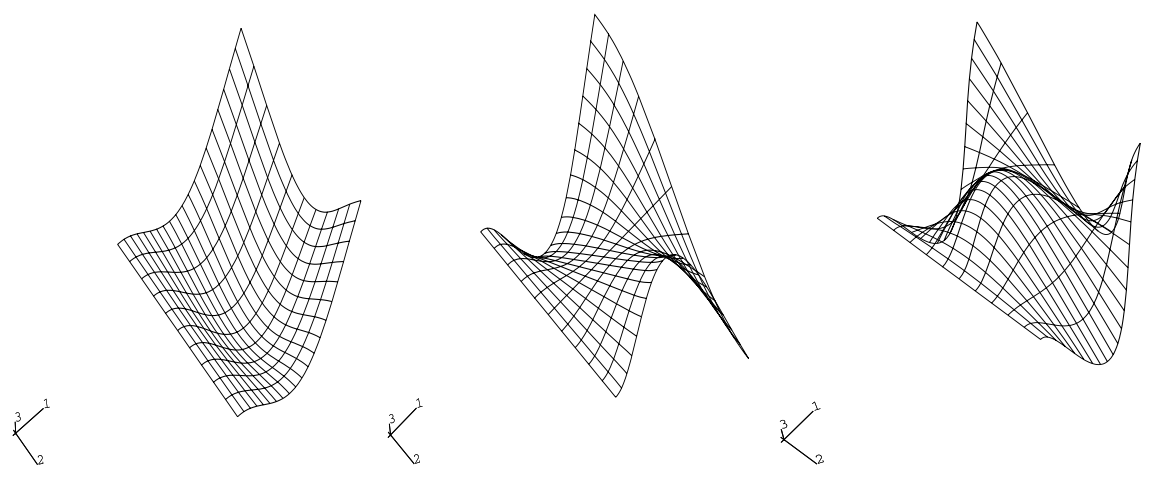

(d)

(e)

(f)

Figure 3.1: The first six mode shapes of the cantilever plate. The 1,2, and 3 directions refer to the $x-, y$-, and $z$ - axes, respectively. 
that the nonlinear coupling was lost and the linear response was obtained. The controller is ineffective in this case. However, when the feedback gain is increased, the controller reduces the vibration amplitude of the plate, as evident from Fig. 3.2(b)-(g). As the gain increases, the steady-state response is decreased, while the transient response becomes more oscillatory. This agrees with the analytical solution discussed in Chapter 2. We also note the downward shift in the curves due to the quadratic control term. In the experiments, this shift needs to be filtered out using an $\mathrm{AC}$ filter, since it was shown to add noise to the system. For excessive values of $\alpha$, the gain effect becomes detrimental and energy is added to the system, as shown in Fig. 3.2(h).

Next, we examine the effect of thickness on the control strategy. For thick plates, the transverse shear-deformation and rotary-inertia effects cannot be neglected. To this end, we compare the results when using a thin-shell element (S9R5) and a thick-shell element (S8R). The formulation for the element S8R is based on the Mindlin theory for thick plates. In Fig. 3.3, we show the results for the plate response using a thin-shell element (Fig. 3.3(a)) and a thick-shell element (Fig. 3.3(b)). In both cases, we use the same values of $\alpha$ and $\gamma$ as 0.075 and 1.0, respectively. As evident from the plots, the effect of thickness is to increase the steady-state solution relative to the no-control (linear) solution. This is due to the fact that a thick plate has a lateral-strain value less than that of a thin plate. This, in turn, reduces the value of the feedback gain $(\alpha)$, and hence results in a larger steady-state response relative to the no-control response. This is consistent with the results of Fig. 3.2, where we study the effect of changing the feedback gain $(\alpha)$. We also note that the oscillatory transient 


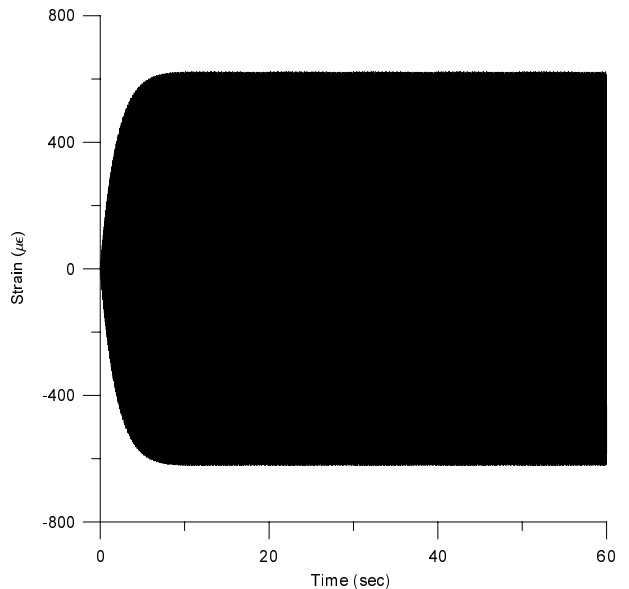

(a)

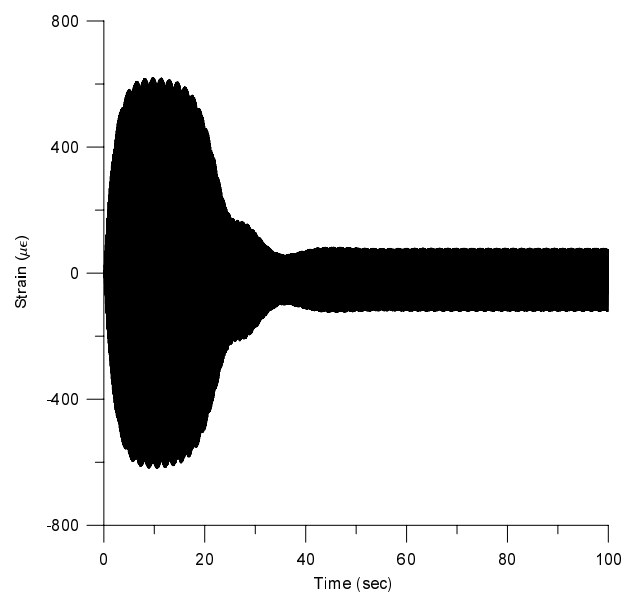

(c)

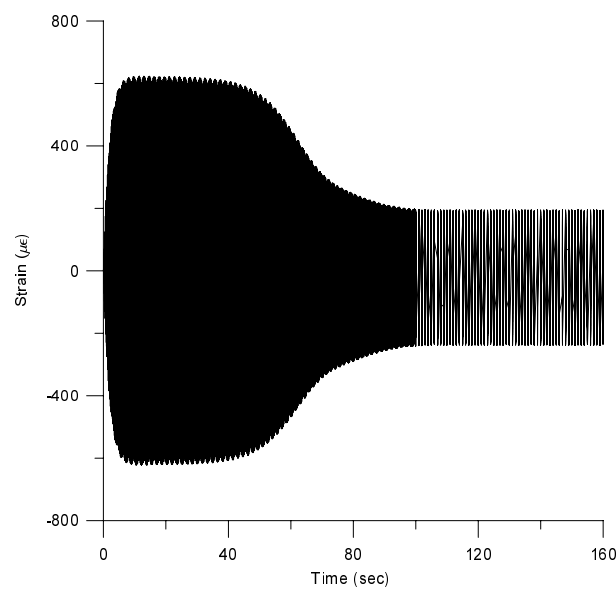

(b)

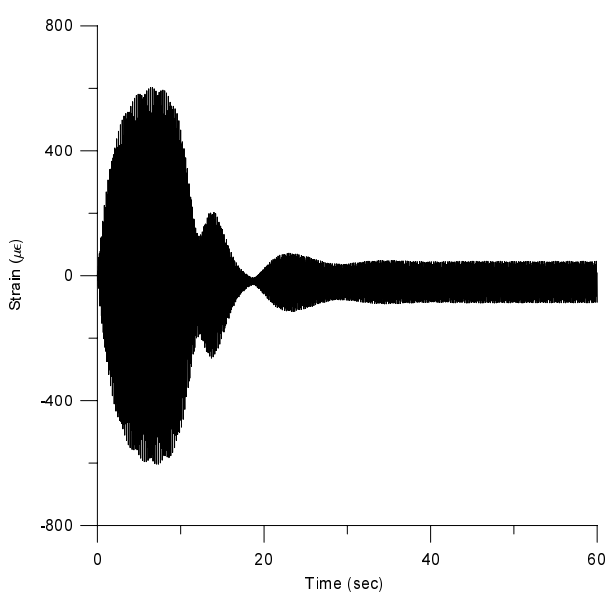

(d)

Figure 3.2: Effect of varying the feedback gain $\alpha$ : (a) $\alpha=0.005$, (b) $\alpha=0.015$, (c) $\alpha=0.06$, (d) $\alpha=0.075$, (e) $\alpha=0.085$, (f) $\alpha=0.09$, (g) $\alpha=0.11$, and (h) $\alpha=0.5$. 


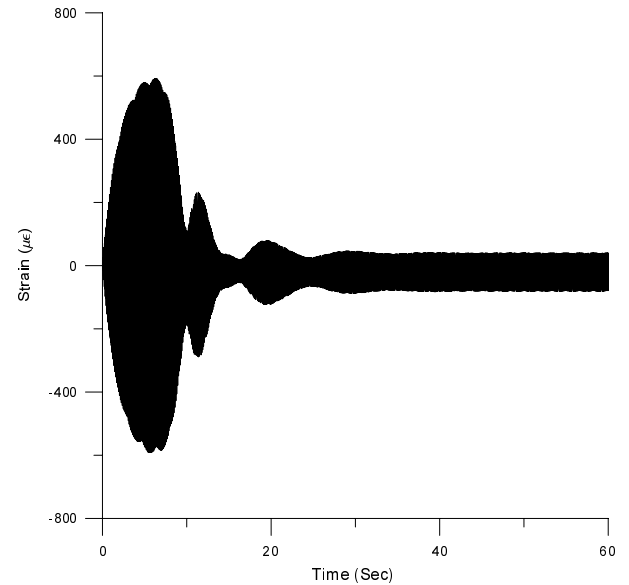

(e)

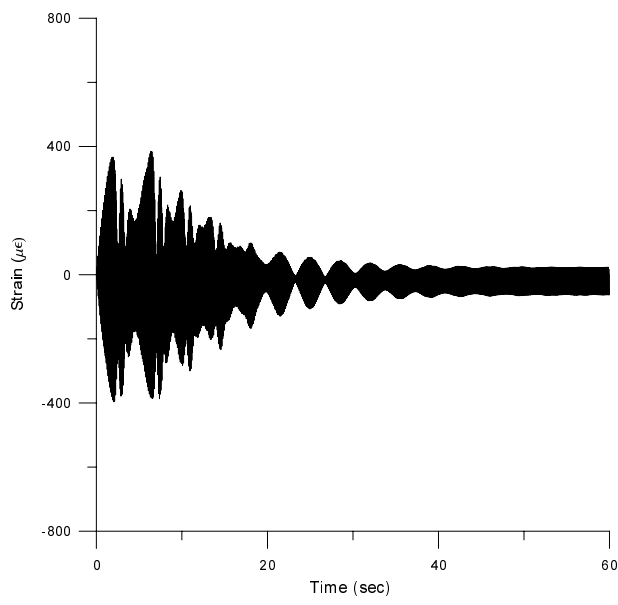

(g)

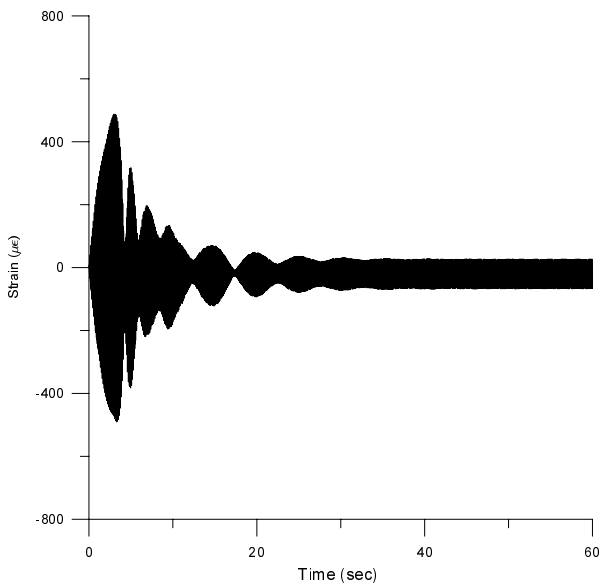

(f)

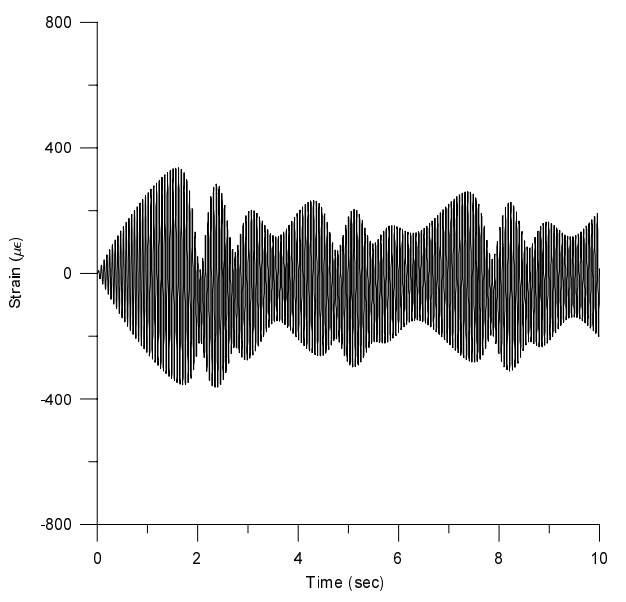

(h)

Figure 3.2(continued): Effect of varying the feedback gain $\alpha$ : (a) $\alpha=0.005$, (b) $\alpha=0.015$, (c) $\alpha=0.06$, (d) $\alpha=0.075$, (e) $\alpha=0.085$, (f) $\alpha=0.09$, (g) $\alpha=0.11$, and (h) $\alpha=0.5$. 
behavior for a thick plate is less than that of a thin one, which is also consistent with the analytical results. We should note here that the relatively larger steady-state response in Fig. 3.3(b) is not due to the less control effort caused by the thickness of the plate, as is the case in linear control. By appropriately increasing the feedback gain $(\alpha)$, one would obtain the same steady-state solution as that of the thin plate.

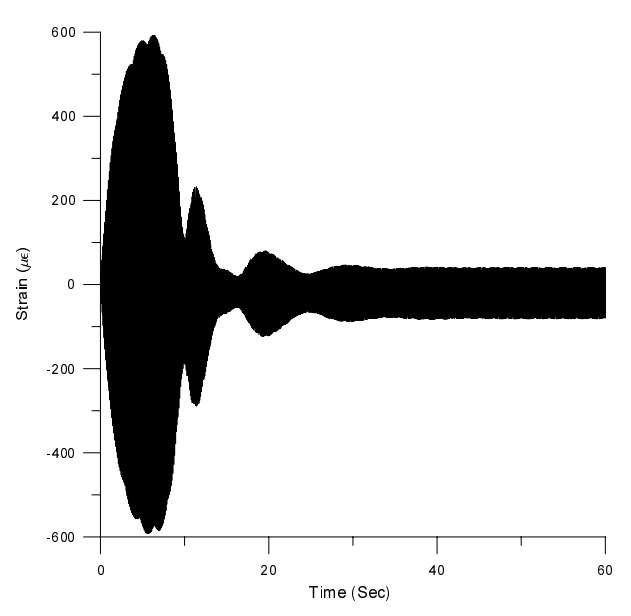

(a)

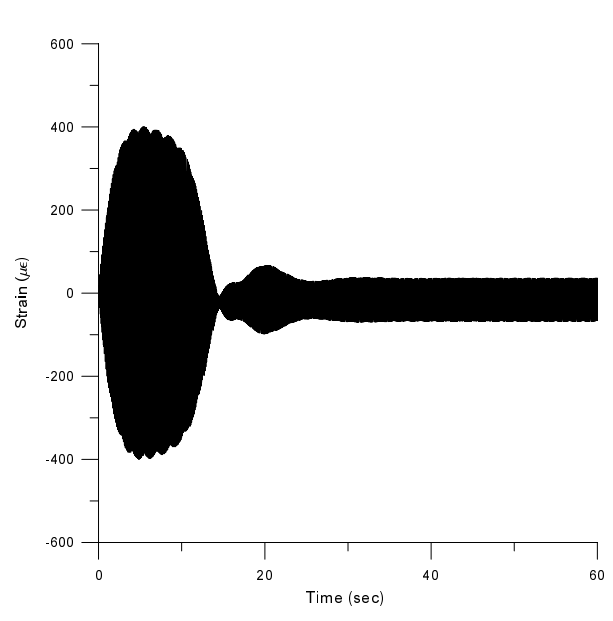

(b)

Figure 3.3: Effect of shear deformation and rotary inertia on the response of the plate: (a) this-shell element and (b) thick-shell element.

\subsection{Modal Analysis}

In this section, we use the mode shapes obtained by ABAQUS to solve the equations of motion (2.35) and (2.38). First, we use the summation of the first five modes to control the response of the first mode. The result is shown in Fig. 3.4, where we plot the time history of the plate displacement at the lower right corner $\left(x=7.5^{\prime \prime}\right.$ and $\left.y=0\right)$. In this case, the 
excitation level is $43 \mathrm{mg}$, the feedback gain is 0.08 , and the control gain is 1 . To examine the effect of the number of modes on the results, we plot in Fig 3.5(a) the response of the second through fifth modes, and in Fig. 3.5(b) the response of the first mode only. Clearly, the response of the first mode is dominant. The quadratic coupling creates a filtering process, such that the controller only affects the mode which is internally resonant with it. All of the other modes decay with time to insignificant levels. Hence, one can use a one-mode analysis without loss of accuracy.

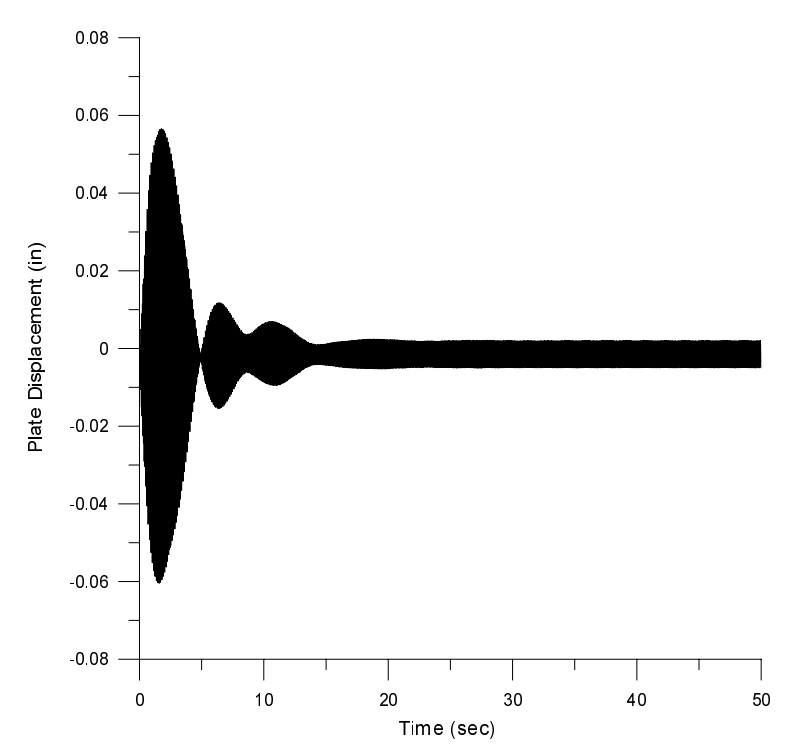

Figure 3.4: Plate displacement at the lower right corner using the first five modes.

Finally, we examine the frequency-response curves. In this regard, we use the shooting method (Nayfeh and Balachandran, 1995) to solve for the steady-state response amplitude and check for the stability of the result. The results are shown in Fig. 3.6. The dashed line represents the unstable linear response, which also coincides with the uncontrolled response. The solid line represents the stable controlled response. Clearly, the saturation phenomenon 


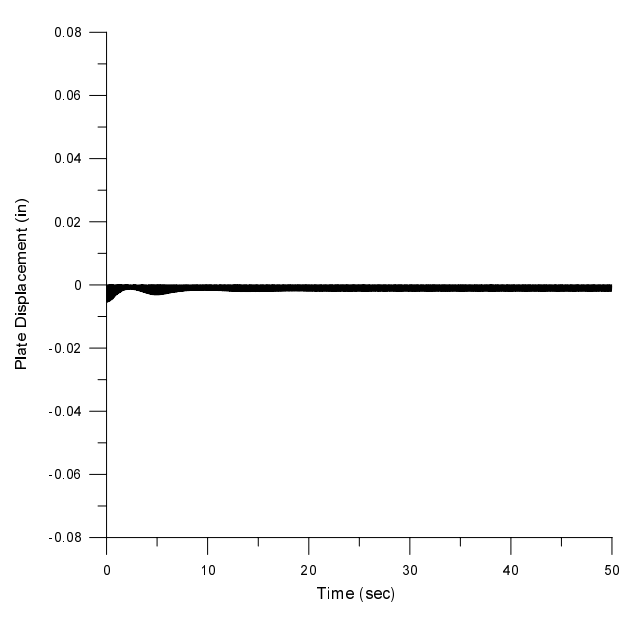

(a)

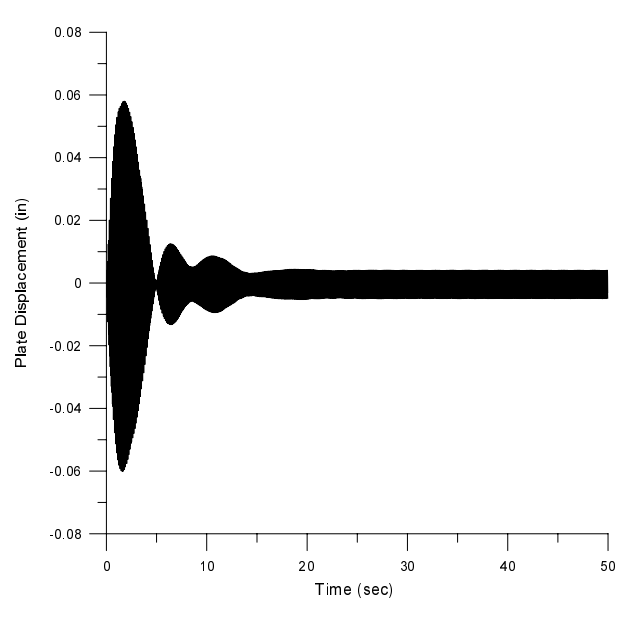

(b)

Figure 3.5: Plate displacement at the lower right corner using (a) the second through fifth modes and (b) the first mode only.

is effective around resonance (which is around $17.9 \mathrm{~Hz}$ ). However, as we move away from resonance, the autoparametric resonance is excited at around $22.5 \mathrm{~Hz}$. This results in another peak in the frequency-response curve. Hence, care must be taken not to excite this resonance. To examine this more, we consider the following system:

$$
\begin{aligned}
\ddot{u}+\xi_{1} \dot{u}+\omega^{2} u & =\gamma v^{2}+F \sin (\Omega t) \\
\ddot{v}+\xi_{2} \dot{v}+\left(\frac{1}{2} \Omega\right)^{2} v & =\alpha u v
\end{aligned}
$$

We assume a solution in the form: $u=A \sin \left(\Omega t+\phi_{1}\right)+\bar{u}$ and $v=B \sin \left(\frac{1}{2} \Omega t+\phi_{2}\right)$, where $\bar{u}$ is a constant resulting from the squaring of $v$. Substituting back for $u$ and $v$ and equating the coefficients of sin and cos, we arrive at

$$
A=\frac{2 F}{\sqrt{\left(3 \omega^{2}-2 \Omega^{2}\right)^{2}+4 \xi_{1}^{2} \Omega^{2}}}
$$


Differentiating equation (3.2) with respect to $\Omega$ and setting the result equal to zero, we obtain the autoparametric resonance condition

$$
\Omega=\sqrt{\frac{3 \omega^{2}-\xi_{1}^{2}}{2}} \approx \sqrt{\frac{3}{2}} \omega
$$

where an assumption of small $\xi_{1}$ has been made. At this frequency, $A=\frac{F}{\xi_{1} \Omega}$.

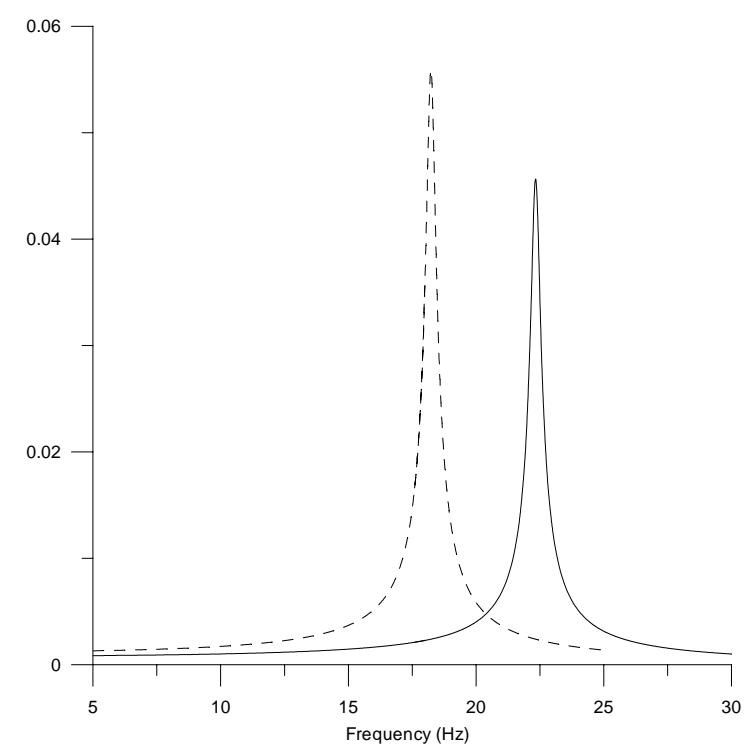

Figure 3.6: Frequency-response curves: (- - ) unstable and (-) stable response.

If, on the other hand, we consider quadratic velocity control instead of quadratic position control, the following system is obtained:

$$
\begin{aligned}
\ddot{u}+\xi_{1} \dot{u}+\omega^{2} u & =-\gamma \dot{v}^{2}+F \sin (\Omega t) \\
\ddot{v}+\xi_{2} \dot{v}+\left(\frac{1}{2} \Omega\right)^{2} v & =\alpha u v
\end{aligned}
$$

where the minus sign is added to $\gamma \dot{v}^{2}$ to ensure that the solution remains bounded (Nayfeh and Mook, 1979). 
In this case, the resonance condition occurs at $\Omega=\frac{\omega}{\sqrt{2}}$. The frequency-response curve for quadratic velocity control is shown in Fig. 3.7, where the dashed line represents the unstable linear solution, while the solid line represents the stable controlled response. In this case, the autoparametric resonance occurs at a lower frequency and farther away from the plate natural frequency. Hence, a choice has to be made in the design of the absorber according to the operation range of frequencies so as to avoid this new resonance.

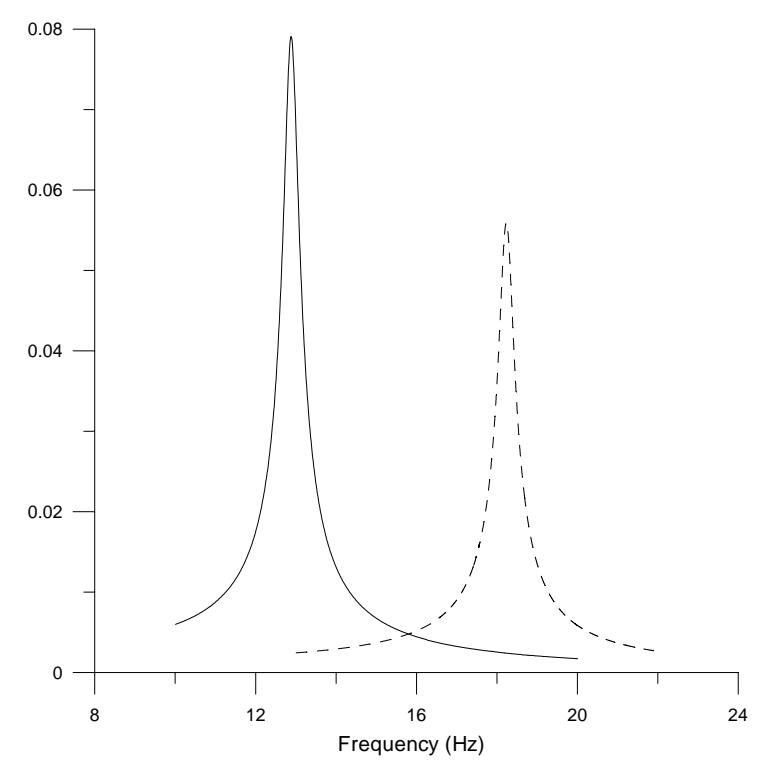

Figure 3.7: Frequency-response curves for quadratic velocity control: (- - -) unstable and $(-)$ stable response. 


\section{Chapter 4}

\section{Optimal Placement of Actuators}

In active vibration control, actuator placement on the structure is a very significant issue. It has a direct effect on the control efficiency and cost. For example, there can be many modes within the frequency band of interest, and this could require many actuators. Also, large flexible structures require many actuators for active vibration control. In these cases, the problem of optimizing the locations of the actuators becomes extremely significant to maximize the system controllability and therefore increase its efficiency. In such cases, the problem of actuator placement becomes more significant than the control law itself. An arbitrary choice of actuator location can seriously degrade the system performance. It can also limit the actuator's range of practical application. If the actuator is placed at the wrong location, the system will require an overly more control force. In this case, the system is said to have a low degree of controllability. To see this more clearly, consider the case in which the actuator is placed on a nodal line for a certain mode of interest. In this case, suppressing this mode becomes impossible, and the system is said to be uncontrollable.

Therefore, our objective is to minimize the control effort and maximize controllability of the 
system. In this work, we present two methods to accomplish this. The first is based on the analytical derivation of the control gain factor presented in Chapter 2. This control gain needs to be maximized over the domain of the system. The second is more general and is based on a global measure of modal controllability for first-order linear systems, which was proposed by Hamdan and Nayfeh (1989).

\subsection{Optimal Placement By Maximizing Control Gain}

The control gain factor given by equation (2.36) needs to be maximized. To this end, we evaluate this control gain over the domain of the plate and search for the maximum value. For a more complicated structure, a search algorithm should be used to find the location at which the degree of controllability is maximum. In this regard, genetic algorithms can be used. In brief, genetic algorithms randomly select initial values of the parameter to be maximized (location of actuators in this case). These initial values are referred to as the initial population. Then, the control gain factor is computed for each member of the population. After that, genetic operators are applied. In this regard, those combinations which produce the largest control gain are kept, while those which produce the lowest control gain are eliminated. These steps are repeated until the desired optimal value is reached.

In this work, we divide the plate into $81 \times 41$ nodes, as we did in the finite-element analysis. The actuator is assumed to have a rectangular area. The length of the actuator extends from $i$ to $i+1$, where $i$ is the node number in the $x$-direction. The width of the actuator extends from $j$ to $j+1$, where $j$ is the node number in the $y$-direction. Then, we evaluate 
the control gain of equation (2.36) at each node on the plate. For each run, the lower-left corner of the actuator is placed at node $(i, j)$. In addition, we use finite-difference formulas to evaluate the involved derivatives of the eigenfunctions. To this end, we use second-order central-, backward-, and forward-difference expressions, which are given, respectively, by

$$
\begin{aligned}
f_{i}^{\prime} & =\frac{f_{i+1}-f_{i-1}}{2 h} \\
f_{i}^{\prime} & =\frac{-f_{i+2}+4 f_{i+1}-3 f_{i}}{2 h} \\
f_{i}^{\prime} & =\frac{3 f_{i}-4 f_{i-1}+f_{i-2}}{2 h},
\end{aligned}
$$

where $f$ is the function that needs to be differentiated, $h$ is the distance between adjacent nodes, and the prime indicates differentiating with respect to $x$. Similar expressions are used for the derivatives with respect to $y$.

We also note that this method does not depend on the parameters of the PZT itself, such as its elastic modulus, piezoelectric constant, and thickness. In other words, it is not a function of $C_{1}$ of equation (2.29). For a particular mode $m$, the maximum control gain depends only on the eigenfunction $\phi_{m}$, whose derivatives need to be evaluated at the boundaries of the actuator.

\subsubsection{Results}

We present results for the optimum locations of the actuator for the first five modes of the plate. Figures 4.1(a)-(e) show plots of the control gain factor $\gamma / C_{1}$ over the domain of the plate. Here, we plot the value of the control gain as we vary the location of the lower left corner of the actuator. From these figures, we can identify the location of the maximum gain 
factor for each mode. We also note that we are interested in the absolute value of this factor. However, if a negative value is chosen, then we have to switch the sign of the feedback gain $\alpha$ to maintain the system stability, as we discussed earlier. From the figures, we generate Table 4.1 for the locations of the maximum gains.

Table 4.1: Locations of maximum controllability

\begin{tabular}{|c|c|}
\hline Mode Number & $(\mathrm{i}, \mathrm{j})$ \\
\hline 1 & $(1,20)$ \\
\hline 2 & $(1,1)$ or $(1,40)$ \\
\hline 3 & $(1,1)$ or $(1,40)$ \\
\hline 4 & $(1,20)$ \\
\hline 5 & $(1,1)$ or $(1,40)$ \\
\hline
\end{tabular}

For the first and fourth modes, the location of maximum controllability corresponds to the middle of the fixed side of the cantilever plate. For the second, third, and fifth modes, this location corresponds to the lower left corner of the plate. For modes 1 and 4 , the location of maximum controllability falls on the symmetry line. For mode 3 , we have two locations of maximum controllability because mode 3 is symmetric. On the other hand, modes 2 and 5 are antisymmetric, and therefore we have two locations of equal absolute values of maximum gain, but with different signs.

In Fig. 4.2(a)-(e), we show the corresponding actuator locations on the plate relative to the nodal lines for each mode. We also indicate in the figures the sign of the maximum gain factor. 


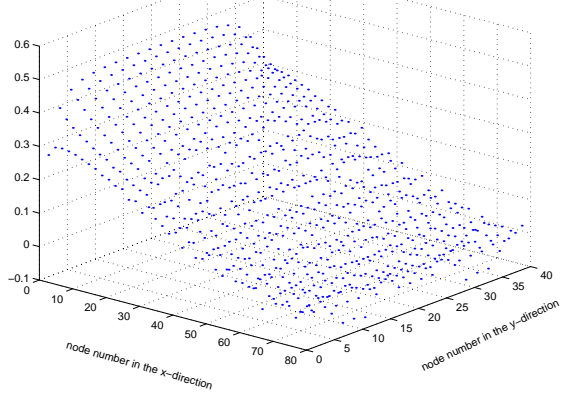

(a)

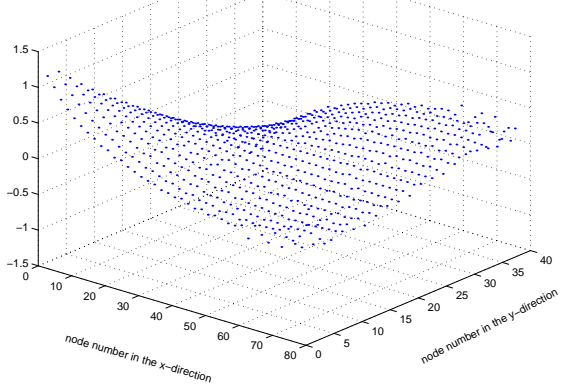

(b)

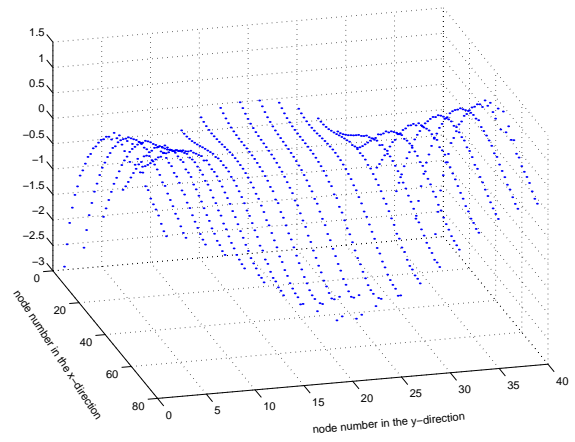

(c)

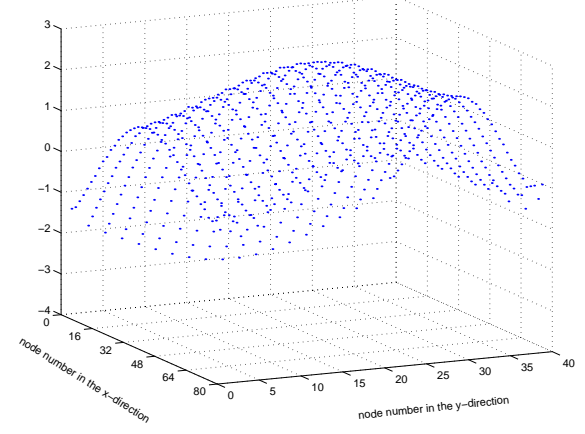

(d)

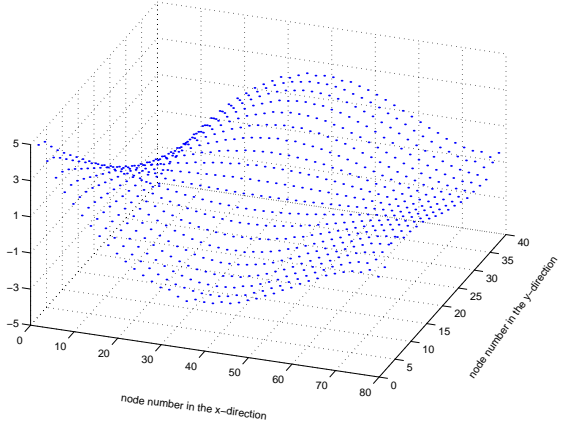

(e)

Figure 4.1: Control gain variation over the domain of the plate for the first five modes: (a) mode 1, (b) mode 2, (c) mode 3, (d) mode 4, and (e) mode 5. 


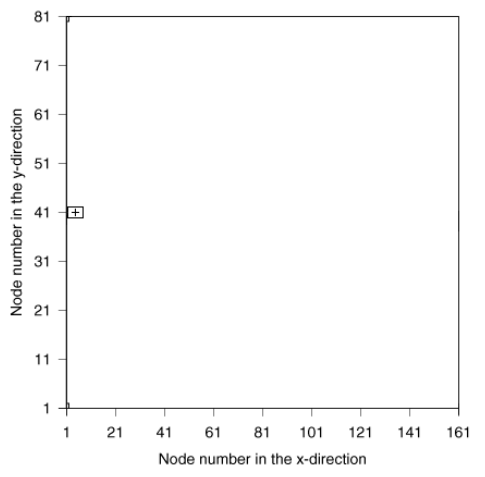

(a)

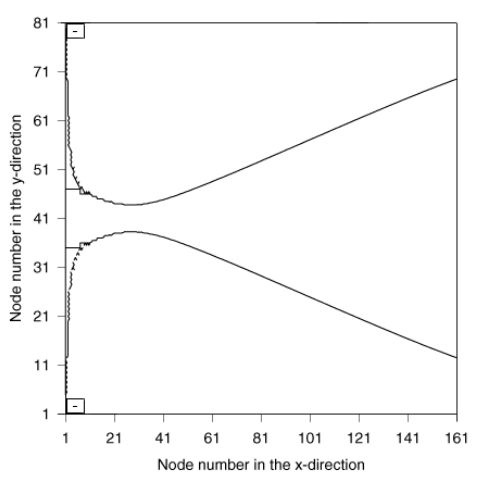

(c)

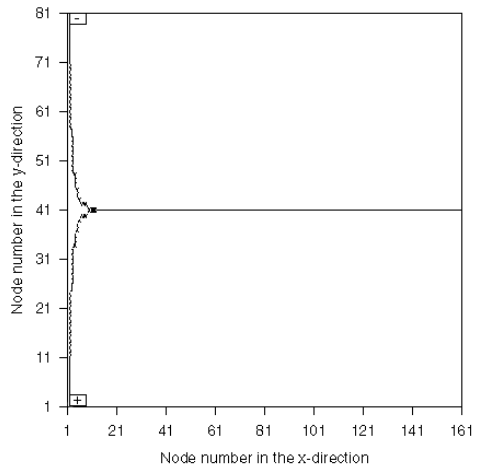

(b)

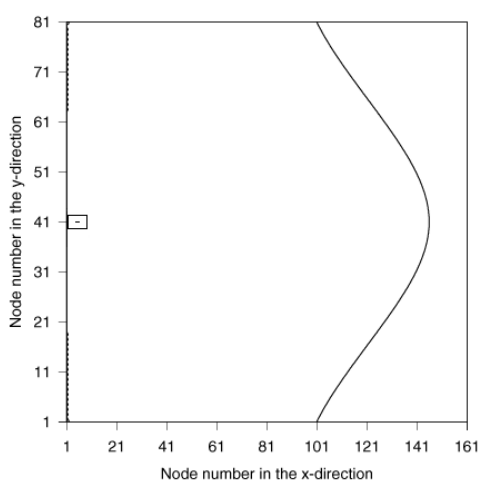

(d)

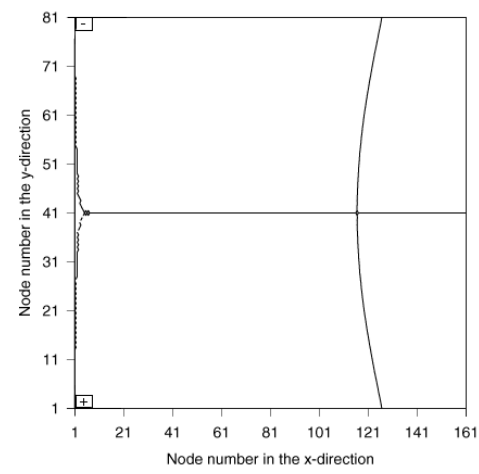

(e)

Figure 4.2: Optimum actuator location on the plate: (a) mode 1, (b) mode 2, (c) mode 3, (d) mode 4, and (e) mode 5. 


\subsection{Optimal Placement Using a Measure of Modal Con- trollability}

In this section, our objective is to exploit a quantitative measure of controllability to optimize the locations of the actuators. Consider a physical structure that is modeled by $n$ discrete modeling mass points. Each modeling point has a one degree of freedom. Hence, we have an $n$-degree-of-freedom mathematical model with $n$ associated modes describing the physical structure. In choosing the modeling points, care should be taken not to locate these modeling points on the nodes of the concerned modes. If possible, these modeling points should be placed at maximum-deflection positions of these modes. Also, the number of these points should be greater than the number of nodal lines along each direction to adequately model the system behavior. Now, consider an $l$ number of actuators acting upon the modeling points. The equations of motion for these modeling points can be written in matrix form as

$$
M \ddot{\mathbf{x}}+C \dot{\mathbf{x}}+K \mathbf{x}=F \mathbf{u}
$$

where $\mathbf{x}$ is an $n \times 1$ displacement vector, $\mathbf{u}$ is an $l \times 1$ input control-force vector, $M, C$, and $K$ are $n \times n$ system mass, damping, and stiffness matrices, respectively, and $F$ is an $n \times n$ matrix that indicates whether an actuator is located at the point or not. Here, we assume all actuators to have the same properties and produce the same actuation force. To locate the optimum actuator location, we place identical actuators on all of the modeling points and measure the controllability from each input. In this regard, we set $l$ equal to $n$ and the matrix $F$ equal to the identity matrix $I$.

The question now is how to obtain the modeling matrices $M, C$, and $K$ for the discrete system 
given by equation (4.2). In this regard, we recall that the eigenfunctions are orthogonal with respect to the mass and stiffness matrices. Furthermore, if the eigenfunctions are normalized with respect to the mass matrix, then we have the following orthogonality conditions with respect to the mass and stiffness matrices, respectively:

$$
\begin{aligned}
& \Phi^{T} M \Phi=I \\
& \Phi^{T} K \Phi=\left[\omega_{n}^{2}\right]
\end{aligned}
$$

where $\Phi$ is the modal matrix whose columns are the eigenfunctions for the $n$ modes and $\left[\omega_{n}^{2}\right]$ is a diagonal matrix of the eigenvalues. Hence, if the eigenfunctions are known, from an experimental FRF analysis or a numerical calculation, the system matrices $M$ and $K$ can be found by manipulating equation (4.3). Thus, we obtain

$$
\begin{aligned}
& M=\left(\Phi \Phi^{T}\right)^{-1} \\
& K=\left(\Phi^{T}\right)^{-1}\left[\omega_{n}^{2}\right] \Phi^{-1}
\end{aligned}
$$

where the superscript -1 indicates matrix inversion. In this work, we use the eigenfunctions obtained from the finite-element package ABAQUS to compute the mass and stiffness matrices. We should note that the real system has an infinite number of modes, while our discrete system is truncated at $n$ modes. This results in nonzero (but small) off-diagonal elements in the matrix $\Phi \Phi^{T}$. This problem is reduced by increasing the number of modes $n$ in our model.

Another significant problem arises when the matrices to be inverted are ill-conditioned, such as the case when one or more rows have very small entries compared to the other rows. In 
this case, the results are inaccurate and matrix inversion should be avoided. Alternatively, the mass matrix can be easily obtained by dividing the total area into $n$ sub-areas and lumping the mass of the sub-areas at the modeling points. Also, the stiffness matrix can be obtained from the influence coefficients. To this end, a unit load is applied at one point of index $i$, and the static deflection at another point of index $j$ is calculated or measured. This gives $1 / k_{i j}$, where $k_{i j}$ is the corresponding element of the stiffness matrix.

The damping matrix $C$ is assumed to be modal and proportional to $M$ and $K$ as follows:

$$
C=\alpha_{1} M+\beta_{1} K
$$

In this work, we take $\alpha_{1}=0.001$ and $\beta_{1}=0$.

\subsubsection{State-Space Model}

The lumped-parameter discrete model described above can now be represented in a statespace model. To this end, we define a state vector $\mathbf{X}$ as

$$
\mathbf{X}=\left[\begin{array}{c}
\mathbf{x} \\
\dot{\mathbf{x}}
\end{array}\right]
$$

Then, equation (4.2) is transferred into a state-space representation as

$$
\dot{\mathbf{X}}=A \mathbf{X}+B \mathbf{u}
$$

where $\mathrm{A}$ and $\mathrm{B}$ are $2 n \times 2 n$ matrices given by

$$
A=\left[\begin{array}{cc}
\emptyset & I \\
-M^{-1} K & -M^{-1} C
\end{array}\right]
$$


and

$$
B=\left[\begin{array}{c}
\emptyset \\
M^{-1} F
\end{array}\right]
$$

Using modal decomposition, we decompose the matrix $A$ as

$$
A=P \Lambda Q^{T}
$$

where $P$ is the modal matrix whose columns are the right eigenvectors $p$ of $A, Q^{T}$ is the modal matrix whose rows are the left eigenvectors $q^{T}$ of $A, \Lambda$ is the diagonal matrix of the eigenvalues of $A$, and the superscript $T$ denotes the transpose of the matrix. The matrices $P, Q$, and $\Lambda$ are found by solving the eigenvalue problem for $A$.

The solution of equation (4.7) for zero-initial conditions is given by (Kailath, 1980)

$$
\mathbf{X}(\mathbf{t})=\int_{0}^{t_{f}} e^{\Lambda(t-\tau)}\left\{Q^{T} B\right\} \mathbf{u}(\tau) d \tau
$$

where $t_{f}$ is the final time. The matrix $\left\{Q^{T} B\right\}$ describes the channel of energy from the input control forces to the modes. This matrix is of extreme importance for determining the controllability of the modes from the control inputs. Until recently, this information was of the yes and no type. If the $(i, j)$ element of the matrix $\left\{Q^{T} B\right\}$ is zero, then the $i$ th mode is uncontrollable form the $j$ th input. This is known as the Popov, Belevitch, and Hautus (PBH) test (Kailath, 1980). The question now is how to obtain quantitative information about the controllability of a certain mode from a certain input. It is not suitable to use the magnitude of $\mathbf{q}_{\mathbf{i}}^{\mathbf{T}} \mathbf{b}_{\mathbf{j}}$, where $\mathbf{b}_{\mathbf{j}}$ is the $j$ th column of $\mathrm{B}$, as a measure of controllability. This is so because the right and left eigenvectors are scaled arbitrarily. 
The magnitude of $\mathbf{q}_{\mathbf{i}}^{\mathbf{T}} \mathbf{b}_{\mathbf{j}}$ can be specified as

$$
\left|\mathbf{q}_{\mathbf{i}}^{\mathbf{T}} \mathbf{b}_{\mathbf{j}}\right|=\left\|\mathbf{q}_{\mathbf{i}}^{\mathbf{T}}\right\| \quad\left\|\mathbf{b}_{\mathbf{j}}\right\| \quad \cos \theta_{i j}
$$

Hmadan and Nayfeh (1989) proposed using $\cos \theta_{i j}$ as a measure of controllability, where $\theta_{i j}$ is the angle between $\mathbf{b}_{\mathbf{j}}$ and $\mathbf{q}_{\mathbf{i}}^{\mathbf{T}}$. When the two vectors $\mathbf{b}_{\mathbf{j}}$ and $\mathbf{q}_{\mathbf{i}}^{\mathbf{T}}$ are orthogonal, the distance between them is maximum and the controllability is zero. On the other hand, when they are parallel, the distance is minimum and the controllability is one. Based on this, we can construct a $2 n \times 2 n$ matrix $\cos \Theta$ whose elements are found from equation (4.10). Each element $\cos \theta_{i j}$ of this matrix is a measure of controllability of the $i$ th mode from the $j$ th input. Thus, the location of maximum controllability for mode $i$ corresponds to the input at location $j$, where $\cos \theta_{i j}$ is maximum.

\subsubsection{Results}

We apply the method described above to locate the optimum actuator locations on the plate. First, we choose 18 modeling points on the plate as shown in Fig. 4.3. The modeling points are numbered and marked by an $\times$. Using these points as nodes in a finite-element analysis, we solve the eigenvalue problem for the first 18 modes using ABAQUS.

The diagonal elements of the mass matrix that are used in the calculations are found to be

$$
\begin{array}{cccc}
m_{1}=1.8975 \times 10^{-5} & m_{2}=9.4875 \times 10^{-6} & m_{3}=1.8975 \times 10^{-5} & m_{4}=9.4875 \times 10^{-6} \\
m_{5}=1.8975 \times 10^{-5} & m_{6}=4.7438 \times 10^{-6} & m_{7}=7.5900 \times 10^{-5} & m_{8}=3.7950 \times 10^{-5} \\
m_{9}=7.5900 \times 10^{-5} & m_{10}=3.7950 \times 10^{-5} & m_{11}=7.5900 \times 10^{-5} & m_{12}=1.8975 \times 10^{-5} \\
m_{13}=1.8975 \times 10^{-5} & m_{14}=9.4875 \times 10^{-5} & m_{15}=1.8975 \times 10^{-5} & m_{16}=9.4875 \times 10^{-5} \\
m_{17}=1.8975 \times 10^{-5} & m_{18}=4.7438 \times 10^{-6} &
\end{array}
$$




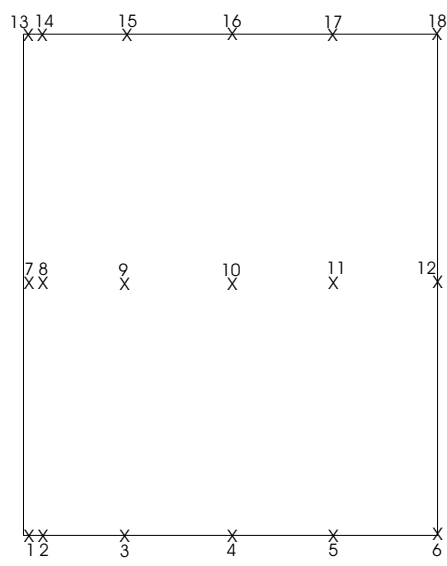

Figure 4.3: Locations of modeling points on the plate for the discrete model.

The stiffness matrix $K$ is given by

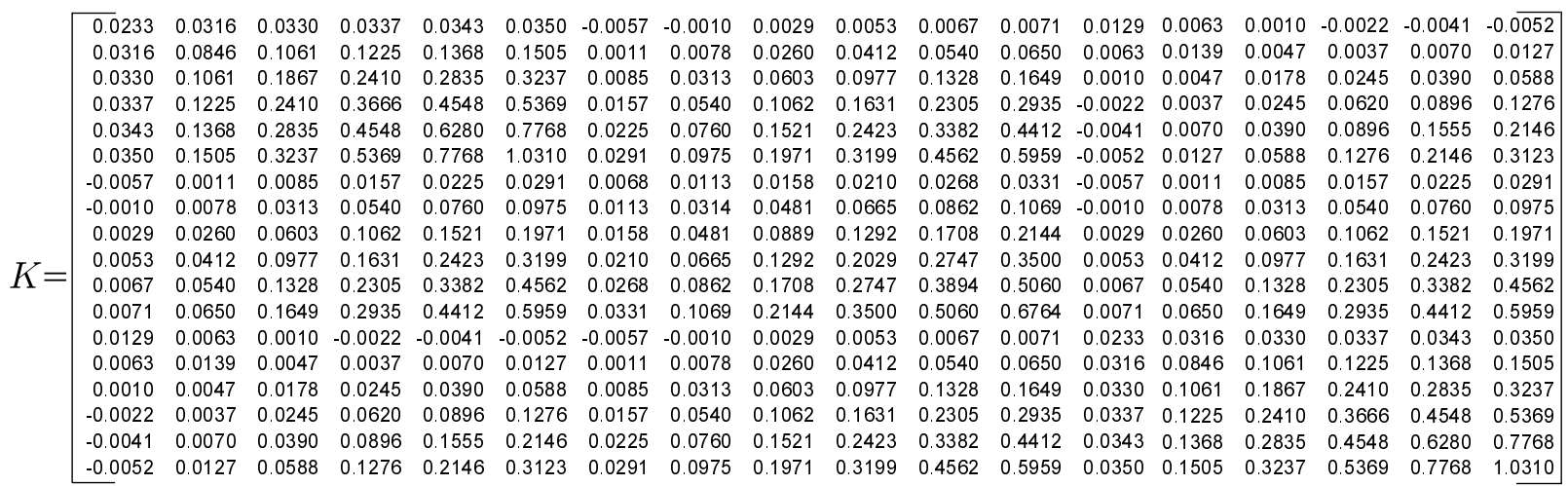

Next, we calculate the controllability measures given by equation (4.10) and construct the

matrix $\cos \Theta$ for the first five modes as

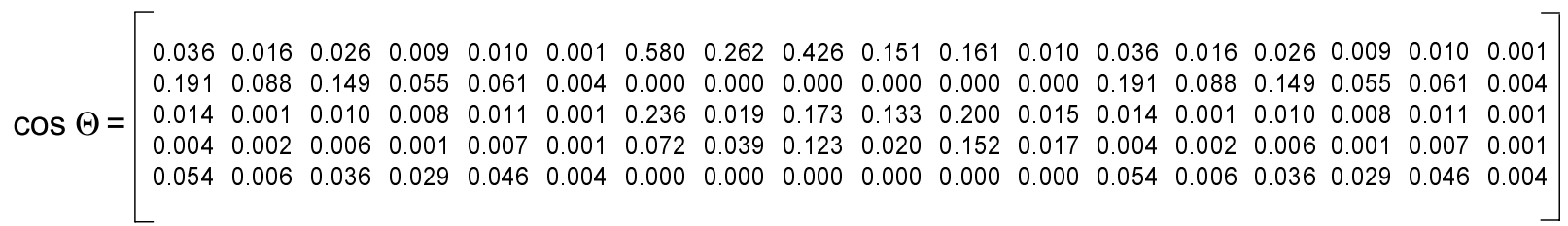


From this matrix, we can identify the optimum actuator location for each mode. Table 4.2 lists these locations for the first five modes. We note that the controllability of the second

Table 4.2: Locations of maximum controllability

\begin{tabular}{|c|c|}
\hline Mode Number & Input Number \\
\hline 1 & 7 \\
\hline 2 & 1 or 13 \\
\hline 3 & 7 \\
\hline 4 & 11 \\
\hline 5 & 1 or 13 \\
\hline
\end{tabular}

and fifth modes from inputs 7 through 12 is zero. This is expected because the actuators are placed on a nodal line in these cases. Also, these modes are antisymmetric and therefore there are two optimum locations. For the other three symmetric modes $(1,3$, and 4$)$, the optimum locations fall on the symmetry lines.

We can extract other useful information about the system controllability from the matrix $\cos \Theta$. For example, denoting by $\mathbf{f}$ an $n \times 1$ vector whose elements are the norms of the rows of $\cos \Theta$, we obtain information about the global controllability of each mode from all of the inputs. Calculating f for our example, we obtain

$$
\mathbf{f}=\left[\begin{array}{l}
0.800 \\
0.383 \\
0.381 \\
0.214 \\
0.121
\end{array}\right]
$$

Hence, mode 1 is the most controllable. Modes 2 and 3 have almost the same global controllability, while mode 5 is the least controllable.

Furthermore, denoting by $\mathbf{h}^{\mathbf{T}}$ a $1 \times n$ vector whose elements are the norms of the columns 
of $\cos \Theta$, we obtain information about the global controllability of all modes from one input. Calculating $\mathbf{h}$ for our example, we obtain

\begin{tabular}{|c|c|c|c|c|c|}
\hline$h_{1}=0.202$ & $h_{2}=0.089$ & $h_{3}=0.156$ & $h_{4}=0.063$ & $h_{5}=0.078$ & $h_{6}=0.006$ \\
\hline$\lambda_{7}=0.630$ & $h_{8}=0.256$ & $h_{9}=0.475$ & $h_{10}=0.202$ & $h_{11}=0.298$ & $h_{12}=0.024$ \\
\hline $13=0.202$ & $h_{14}=0.089$ & $h_{15}=0.156$ & $h_{16}=0.063$ & $h_{17}=0.078$ & $h_{18}=0.006$ \\
\hline
\end{tabular}

From this, we deduce that the input at point number 7 gives the highest global controllability for the first five modes. Locations 6 and 18, which correspond to the lower and upper right corners of the plate, respectively, have the lowest measure of global controllability. Hence, these locations should be avoided. 


\section{Chapter 5}

\section{Experimental Setup and Results}

We present experimental tests conducted to verify the effectiveness of the control strategy. Furthermore, a parametric study is conducted to relate the effect of changing the controller parameters on the response of the plate. Here, we are concerned with the effect of the nonlinear gains: the feedback gain $\alpha$ and the control gain $\gamma$. Other significant parameters include the controller damping coefficient and initial conditions. A frequency-response curve is constructed to illustrate the broadband effectiveness of the controller. In addition, a forceresponse curve is generated to verify the saturation phenomenon for systems with quadratic nonlinearities and a two-to-one internal resonance. Three sets of experiments are presented. The first one relates to the control of vibrations of the first mode (first bending mode). The second set relates to the control of the second mode (first torsion mode). Finally, in the third set of experiments, we present the results for simultaneously controlling the first two modes of the plate. 


\subsection{Setup and Procedure}

We perform the tests on an aluminum plate with dimensions $9.00^{\prime \prime} \times 7.50^{\prime \prime} \times 0.03^{\prime \prime}$. A fixture is used to hold stationary one edge of the plate. The plate is attached to a 100-lb permanent magnet shaker. The shaker moves in the vertical direction and is driven by a power amplifier and a signal generator, which drives the shaker at the desired frequency and amplitude. Two patches of piezoelectric ceramics (Lead-Zicronate-Titanate) are used as actuators. The dimensions of the PZT patches are $1.3^{\prime \prime} \times 0.75^{\prime \prime} \times 0.0075^{\prime \prime}$. The PZTs are activated by a piezoamplifier, which amplifies the input voltage signal by a factor of 20 with a maximum of $10 \mathrm{~V}$ input limit. The actuators are attached to the cantilever plate away from the nodal lines. This is so because controllability is zero at these lines. The results of Chapter 4 for optimizing the locations of the actuators are utilized in these experiments. For the first-mode experiments, the nodal line is clearly identified at the root of the plate. For the second-mode vibrations, we also use a stroboscope to experimentally identify the nodal line. The results agree with the finite-element results using ABAQUS.

A strain gage is attached very close to the fixed edge of the plate at a distance $y=2.16^{\prime \prime}$ from the lower left corner. The strain gage is used to measure the response of the plate as well as to provide the controller feedback signal. Changes in the gage are measured with a conditioning amplifier that uses a quarter bridge configuration. The output voltage signal from the strain gage conditioning amplifier is converted into strain using the following 
relation:

$$
\mu \epsilon=\frac{4 V_{\text {out }}}{10 \times 1000 \times 2.12 \times 10^{-6}}
$$

where the bridge excitation is 10 , the amplifier gain is 1000 , the gage factor is 2.12 , and $\mu \epsilon$ is the strain in microns. In the following experiments, the signal from the strain gage is amplified by a factor of $20 \mathrm{~dB}$ after filtering. This is done in order to increase the signal-tonoise ratio. The base excitation of the shaker is monitored by an accelerometer attached to the fixture clamping the edge of the plate.

The experimental setup is shown in Fig. 5.1. The strain gage signal from the conditioning amplifier is fed to the controller circuit, and the output control signal from the controller circuit is filtered, amplified, and sent to the actuators. The response of the plate is monitored by an oscilloscope and a four-channel HP signal analyzer. A data acquisition card and a PC are used to collect the data for plotting.

To measure the natural frequencies of the plate, we first conduct an FRF analysis, where a random base excitation is used. This provided a rough estimate of the natural frequencies. A fine-tuning process is then conducted to accurately locate the natural frequencies. The strain gage signal is then fed to the signal analyzer, and the peaks are identified to be the natural frequency locations. For the tested plate, the first two natural frequencies are measured to be at $17.9 \mathrm{~Hz}$ and $36.8 \mathrm{~Hz}$, respectively. 


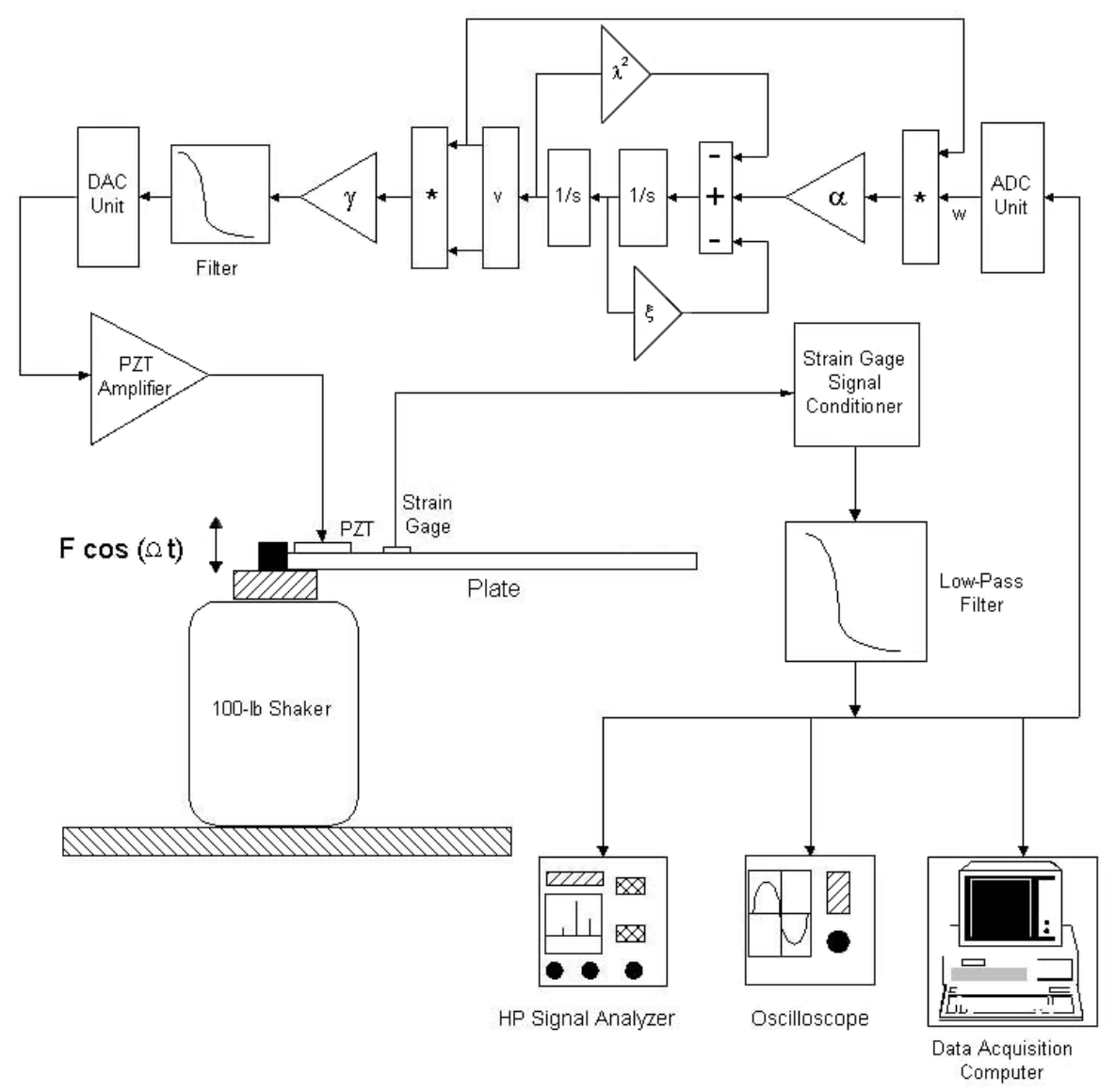

Figure 5.1: The experimental setup.

\subsection{Controller Circuit}

As a controller, we use a digital control system, which utilizes the modeling software SIMULINK and the dSPACE DS1103 controller installed in a PC. First, we use the SIMULINK software to build the control block diagram. This SIMULINK model is then used to generate a C-code model by the dSAPCE Real-Time Workshop. The dSPACE Real-Time Interface then connects this code with the dSPACE hardware for simulation. The block diagram of the controller is also shown in Fig. 5.1. The filter used is a Butterworth IIR low-pass filter. 
The controller frequency is set at one-half the value of the plate natural frequency to exploit the saturation phenomenon.

\subsection{Results}

\subsubsection{Control of the First Mode}

First, we examine the results for controlling the first mode of vibration. We subject the plate to a base excitation of $11.95 \mathrm{mg}$ at the first natural frequency $f=17.9 \mathrm{~Hz}$. In Fig. 5.2, the plate response is shown when the controller was switched on at $t \approx 5 \mathrm{sec}$. The controller is then left on until $t \approx 40 \mathrm{sec}$. At that time, the controller is switched off and the uncontrolled plate response is restored. The nonlinear gains in this case are set at $\alpha=0.05$ and $\gamma=1$. We give the controller an initial displacement of $0.1 \mathrm{~V}$ and a zero initial velocity. In Fig. 5.3, the controller is activated simultaneously with the shaker. The saturated steady-state response is obtained at $t \approx 40 \mathrm{sec}$. It is evident that the control method is very effective in reducing the vibration level very quickly and eventually eliminating it. The plate response is reduced by $31.7 \mathrm{~dB}$.

To study the effect of varying the nonlinear feedback gain $\alpha$, we set the gain level at 0.01 , 0.05, and 0.12. The results are shown in Fig. 5.4. All other controller conditions (control gain and initial conditions) are the same as those in Fig. 5.2. Clearly, increasing $\alpha$ reduced the steady-state controlled response. In addition, it reduced the time needed to reach steady state and, hence, the response could be reduced significantly within a shorter time. However, the transient response is oscillatory. These results agree with our findings using perturbation 


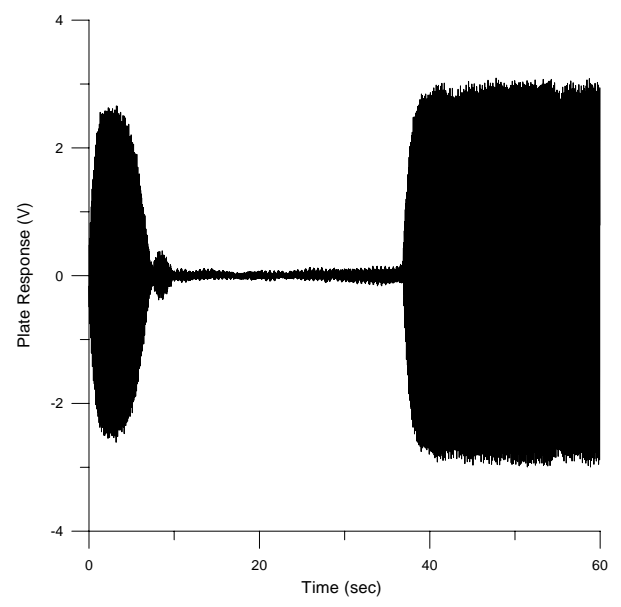

Figure 5.2: Control of the first mode of the plate.

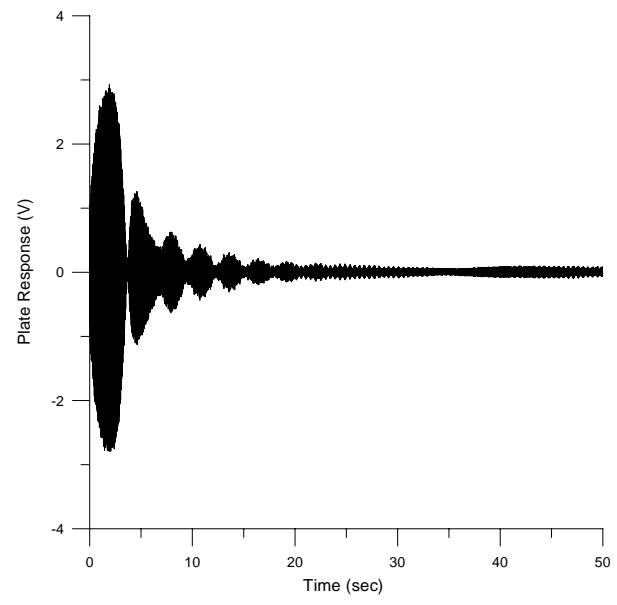

Figure 5.3: The plate response when $\alpha=0.1$ and $\gamma=1$.

methods, as discussed in Chapter 2, where we show that the response is proportional to $\xi / \alpha$. Using perturbation methods, it is also shown that beyond a critical value of $\alpha$, the transient response becomes oscillatory, indicating a stable focus solution. These results also agree with the numerical results using ABAQUS discussed in Chapter 3.

Next, we study the effect of varying the control gain $\gamma$. The values of $\gamma$ are set at $1.0,0.5$, and 


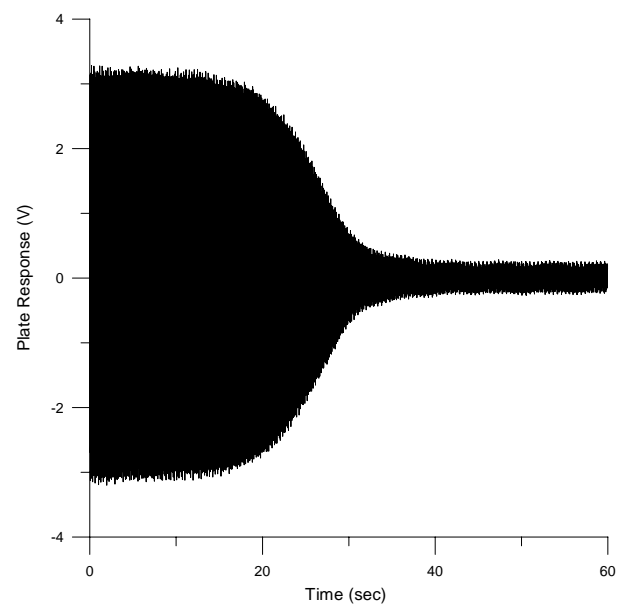

(a)

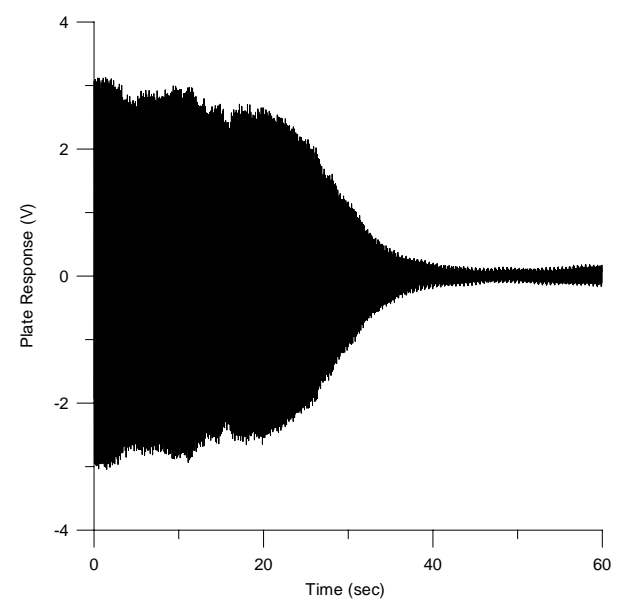

(b)

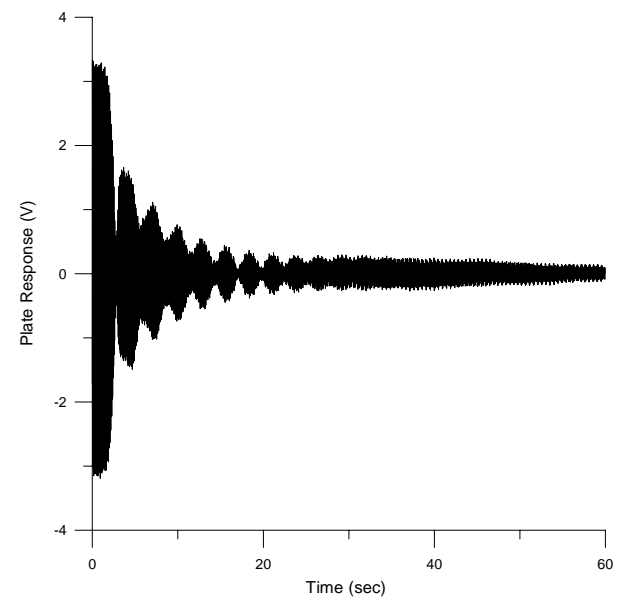

(c)

Figure 5.4: Effect of varying the nonlinear feedback gain $\alpha$ : (a) $\alpha=0.01$, (b) $\alpha=0.05$, and (c) $\alpha=0.12$.

0.25. All other conditions correspond to those of Fig. 5.2. As the control signal changes, the steady-state plate response is almost unchanged. However, the controller response is seen to be inversely proportional to the control gain, as is evident from Fig. 5.5. Again, this agrees with the analytical findings using perturbation methods, where the controller response is 
shown to be inversely proportional to $\gamma$.

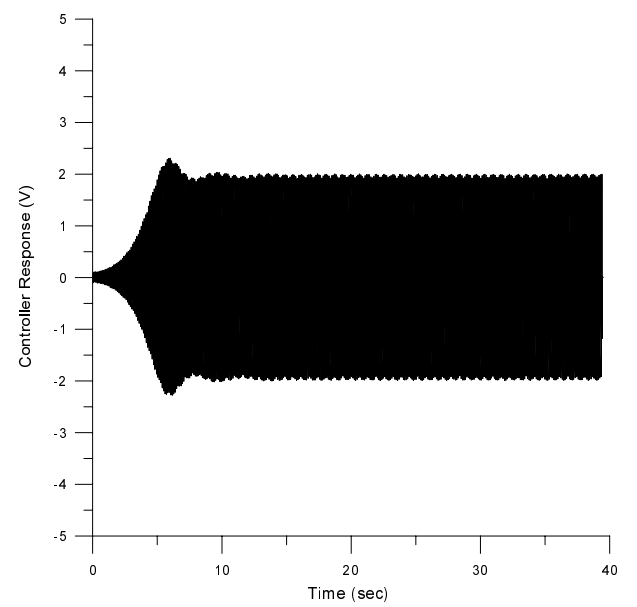

(a)

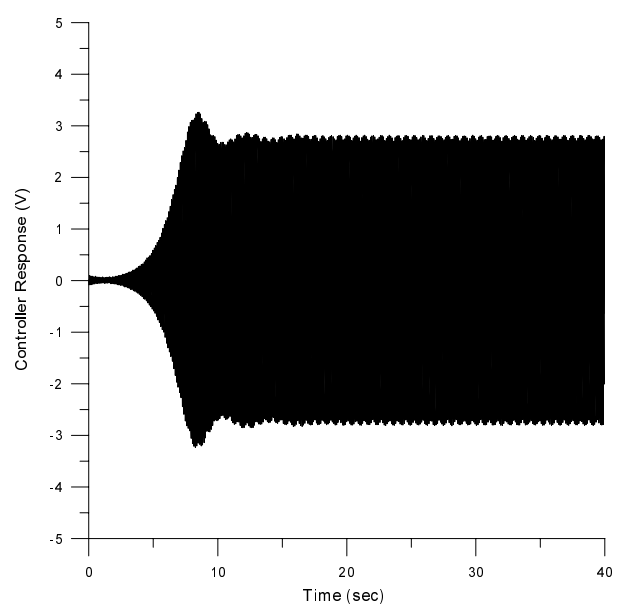

(b)

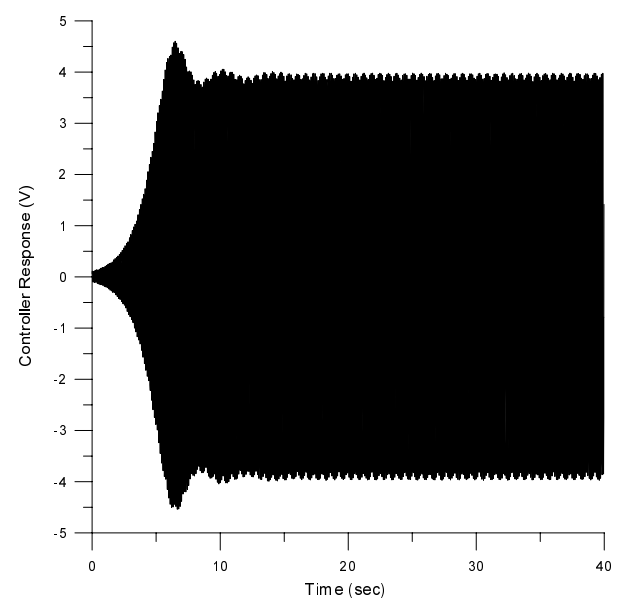

(c)

Figure 5.5: Effect of varying the nonlinear control gain $\gamma$ : (a) $\gamma=1.0$, (b) $\gamma=0.5$, and (c) $\gamma=0.25$.

The effect of the controller initial conditions is studied next. In Fig. 5.6, we vary the initial controller displacement. Four values are chosen at $0.5,1.0,1.6$, and $2.0 \mathrm{~V}$. The gains are set at $\alpha=0.03$ and $\gamma=1$. The initial controller velocity is set at zero. From 
the figure, we can see that there is an optimum initial displacement at which the transient response is minimized. The initial displacement value of $1.6 \mathrm{~V}$ is found to be optimum in this regard. Beyond this value, the response is even amplified during the transient phase. In this case, energy is added to the system detrimentally. In Fig. 5.7, we set the initial controller displacement at $1.6 \mathrm{~V}$ and vary the initial controller velocity to 0.5 and $1.0 \mathrm{~V} / \mathrm{sec}$. Clearly, a non-zero initial velocity detrimentally affects the behavior by amplifying the transient response and delaying the steady-state solution.

We conclude the parametric study by investigating the effect of changing the controller damping coefficient $\xi$. Three values of the damping coefficient are chosen: $\xi=0.01,0.05$, and 0.1. The results are shown in Fig. 5.8. Clearly, increasing the value of $\xi$ reduces the control effect. The steady-state amplitude increases by increasing the damping coefficient. Again, this agrees with the perturbation solution, where the response is proportional to $\xi / \alpha$.

Finally, we conclude the first set of experiments by discussing the results for the frequencyresponse and force-response curves. In Fig. 5.9, frequency-response curves are shown for both the open-loop (controller off) and closed-loop (controller on) response. Clearly, the control strategy is effective for a wide range of frequencies around the resonance frequency. While the open-loop response has a peak at $f_{n} \approx 17.9 \mathrm{~Hz}$, the closed-loop response remains virtually constant at a negligible value. Force-response curves are shown in Fig. 5.10. Here, the saturation phenomenon is clearly manifested. The open-loop response is almost linear as expected, while the closed-loop response saturates at $F \approx 7.3 \mathrm{mg}$. Beyond this value, the response is independent of the forcing amplitude. All of the energy added to the system 


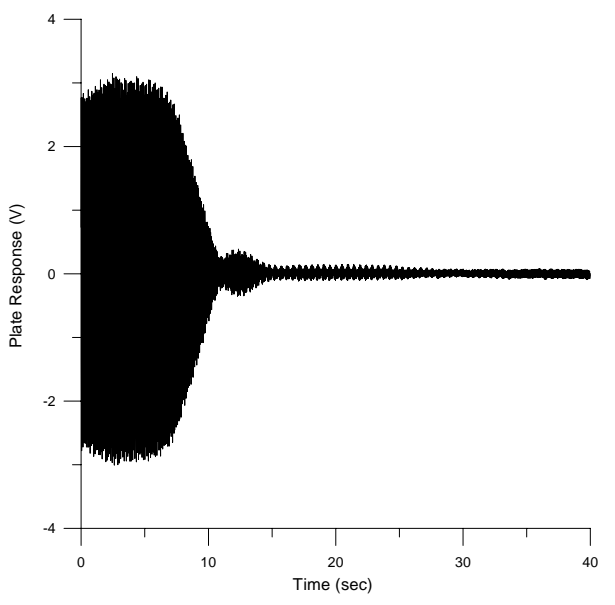

(a)

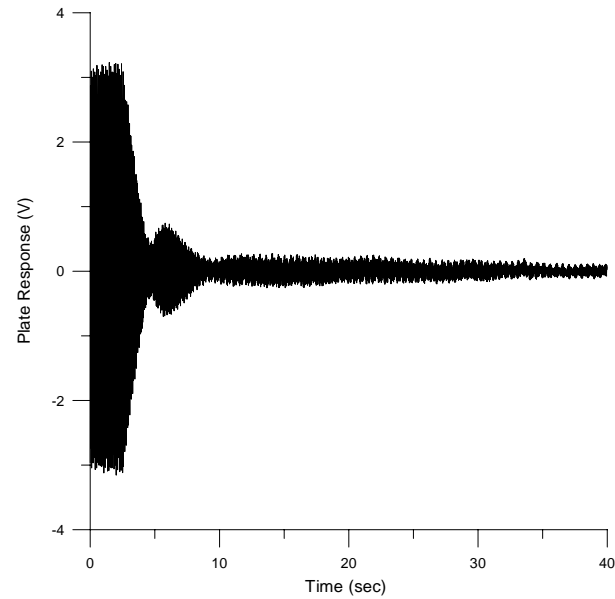

(c)

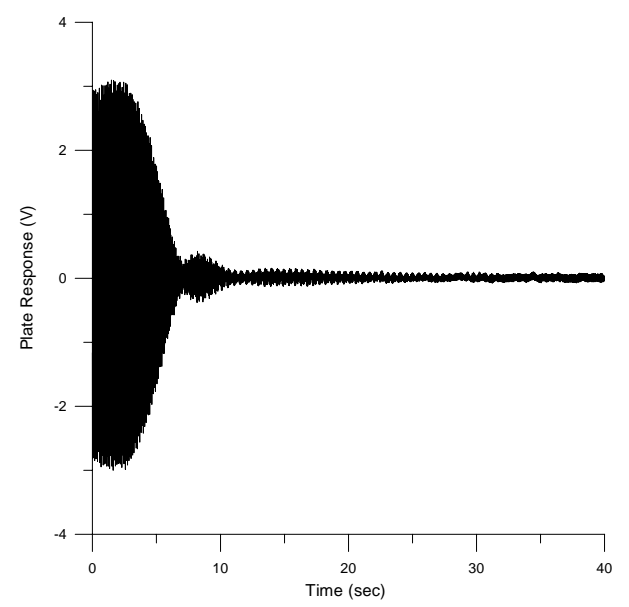

(b)

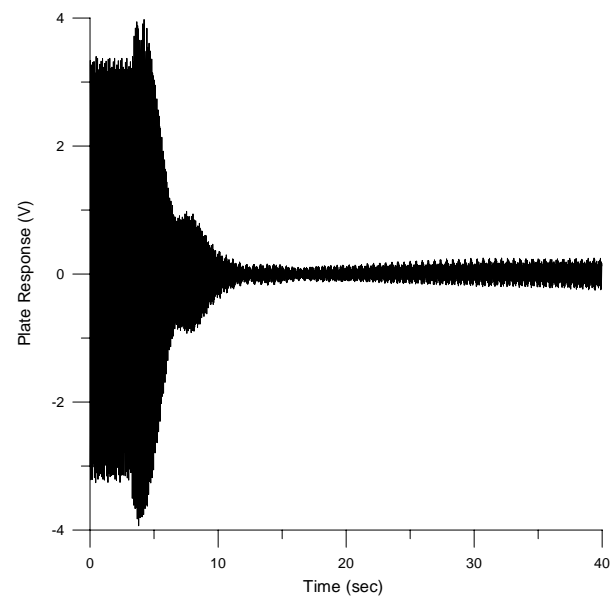

(d)

Figure 5.6: Effect of varying the initial controller displacement: (a) $v=0.5 \mathrm{~V}$, (b) $v=1.0 \mathrm{~V}$, (c) $v=1.6 \mathrm{~V}$, and (d) $v=2.0 \mathrm{~V}$.

is effectively channeled by the controller to the actuator, which is used to suppress the vibration. 


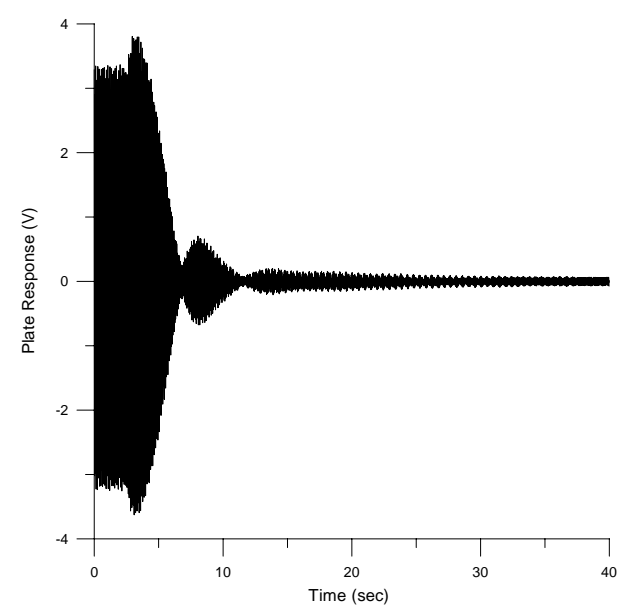

(a)

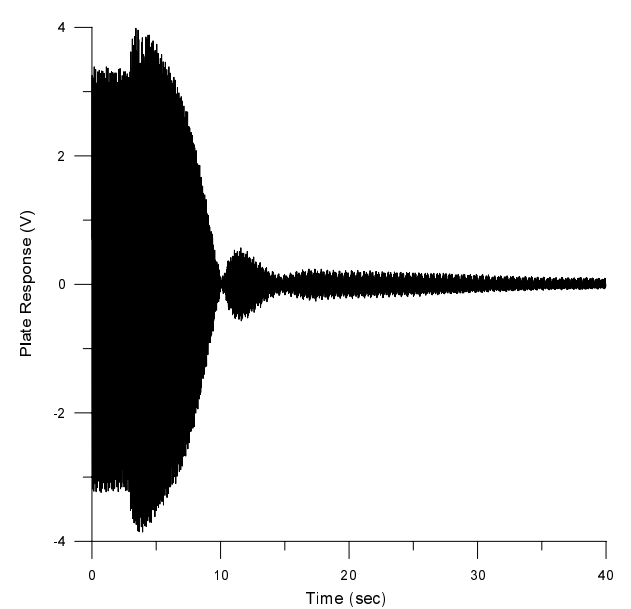

(b)

Figure 5.7: Effect of varying the initial controller velocity: (a) $\dot{v}=0.5 \mathrm{~V} / \mathrm{sec}$ and (b) $\dot{v}=$ $1.0 \mathrm{~V} / \mathrm{sec}$.

\subsubsection{Comparison with the Numerical Results}

To compare our experimental and numerical results, we choose the case $\alpha=0.075, \gamma=1.0$, and $F=15 \mathrm{mg}$. In Fig. 5.11, we plot the steady-state experimental results together with the numerical response obtained from ABAQUS. We remove the DC offset in the numerical results and integrate the results for sixty seconds to obtain the steady-state response. As evident from the figure, the agreement is very good.

\subsubsection{Control of the Second Mode}

In the second set of experiments, we study the effectiveness of the control strategy in controlling the first torsional mode. The plate is subjected to an excitation amplitude of $1.12 \mathrm{~V}$. In Fig. 5.12, we show both the plate response and the controller output for the gain values 


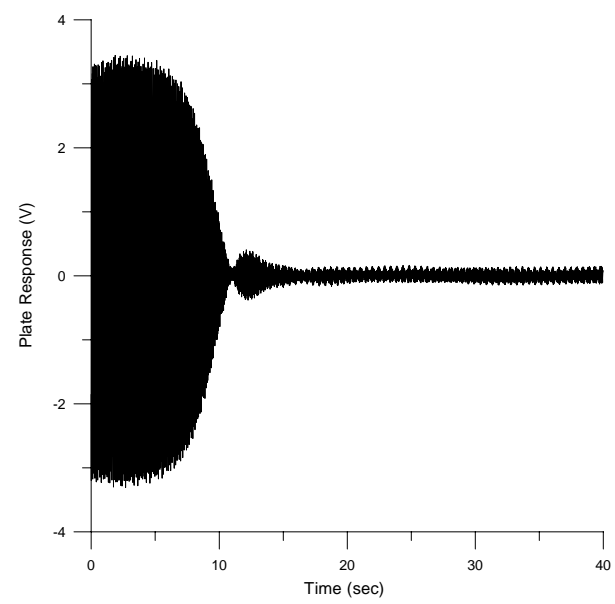

(a)

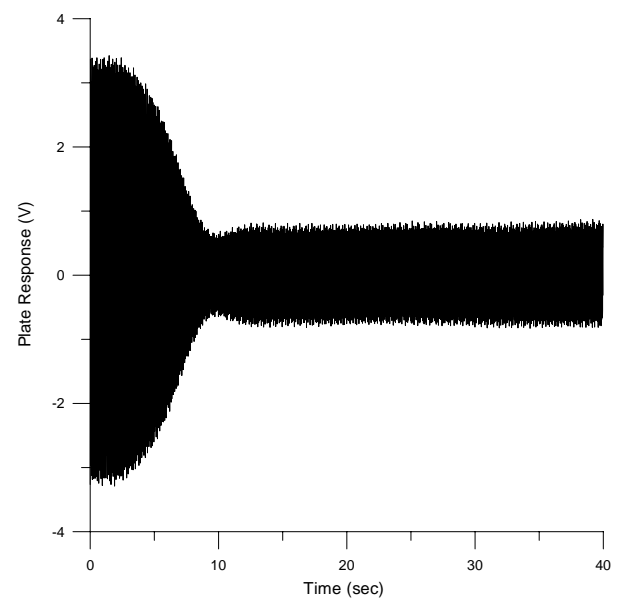

(b)

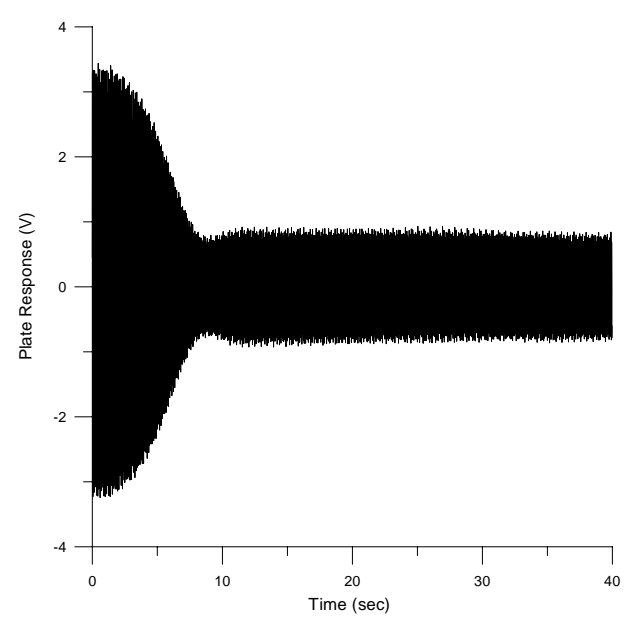

(c)

Figure 5.8: Effect of varying the controller damping coefficient $\xi$ : (a) $\xi=0.01$, (b) $\xi=0.05$, and $(\mathrm{c}) \xi=0.1$.

of $\alpha=0.03$ and $\gamma=0.1$. The initial conditions are set at a displacement of $0.3 V$ and a zero velocity. The plate response is reduced by $45 \mathrm{~dB}$ at steady state.

In Fig. 5.13, we study the effect of varying the feedback gain $\alpha$. To this end, we set the 


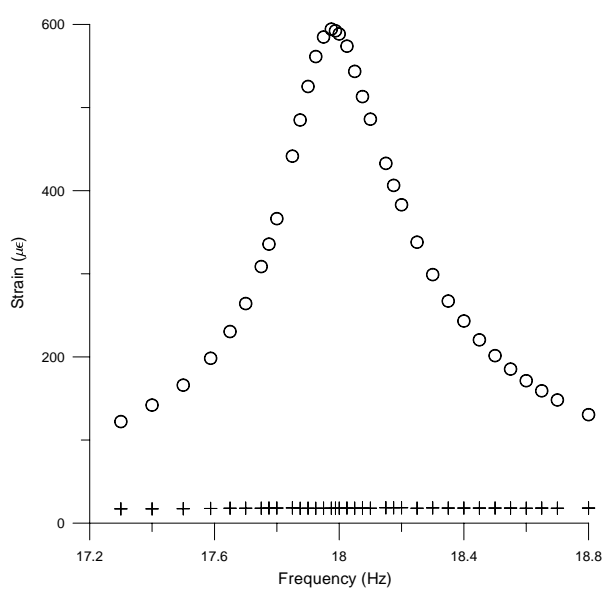

Figure 5.9: Frequency-response curves: (ooo) controller off and $(+++)$ controller on.

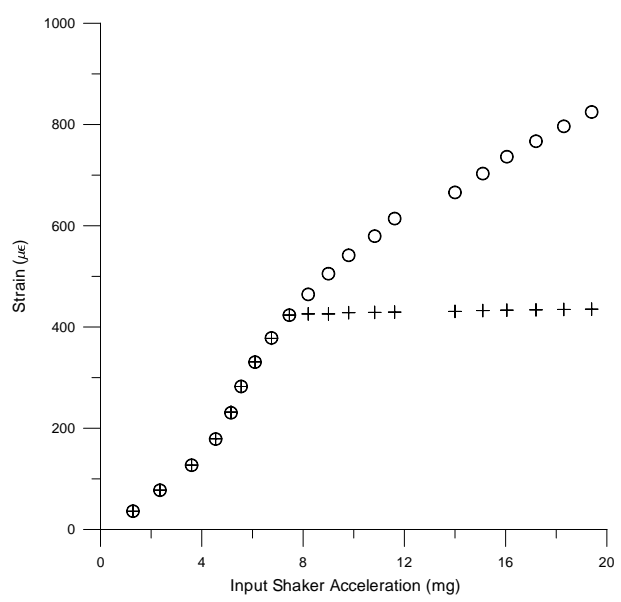

Figure 5.10: Force-response curves: (ooo) controller off and $(+++)$ controller on.

value of $\alpha$ at $0.04,0.07,0.1,0.11,0.12$, and 0.15 . For $\alpha<0.1$, increasing the gain has an advantageous effect on the control effort. While the steady-state response is almost constant at a negligible value $(-46 \mathrm{~dB})$, the time to reach steady state is significantly reduced from around $100 \sec$ when $\alpha=0.04$ to around $50 \sec$ when $\alpha=0.1$. For values of $\alpha$ larger than 0.1, increasing the gain seems to have a detrimental effect on the result. Energy is added to the system and the response amplified. This agrees with the numerical results of Chapter 3. 


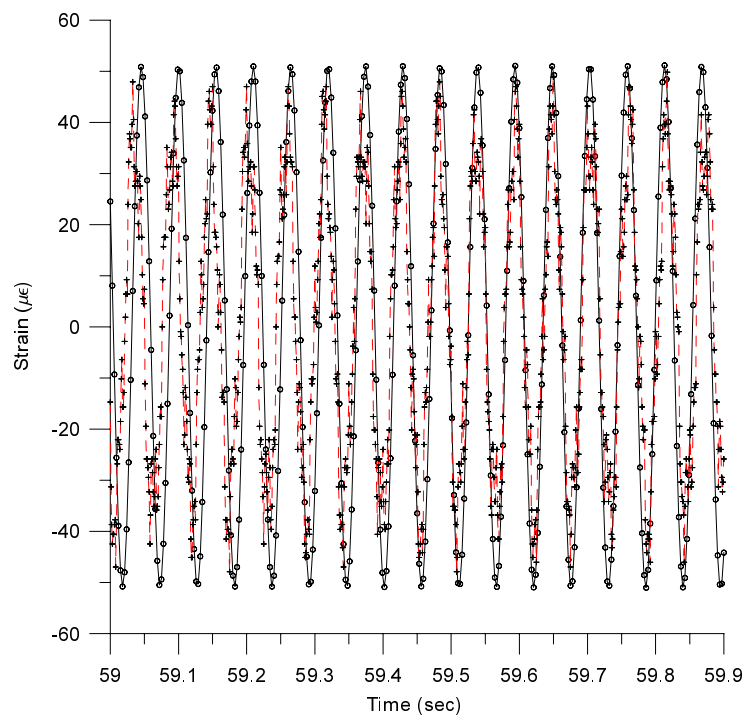

Figure 5.11: Plate response when $\alpha=0.075, \gamma=1.0$, and $F=11.9 \mathrm{mg}:(+++)$ experimental results and (ooo) numerical results.

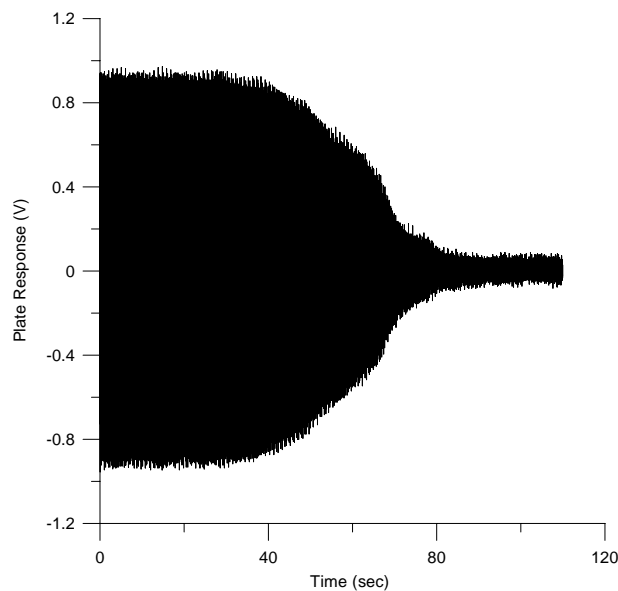

(a)

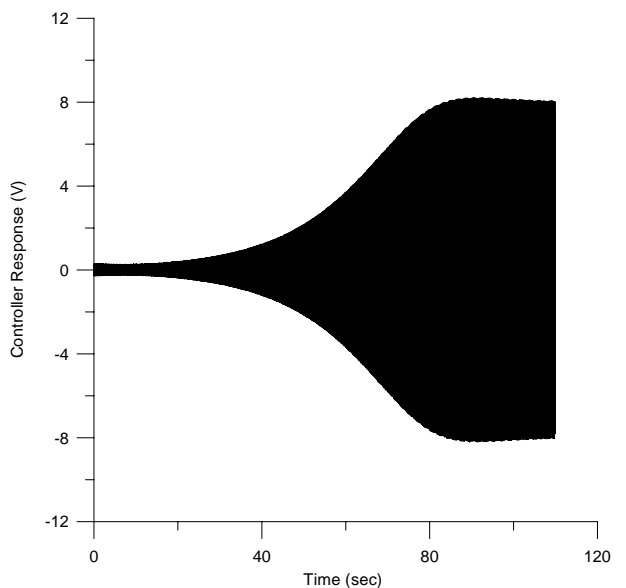

(b)

Figure 5.12: Control of the second mode of the plate: (a) plate response and (b) controller response.

Next, we study the effect of changing the initial displacement of the controller. The three values of initial displacement of $0.03,3.0$, and 5.0 are chosen. Clearly, the time to reach 


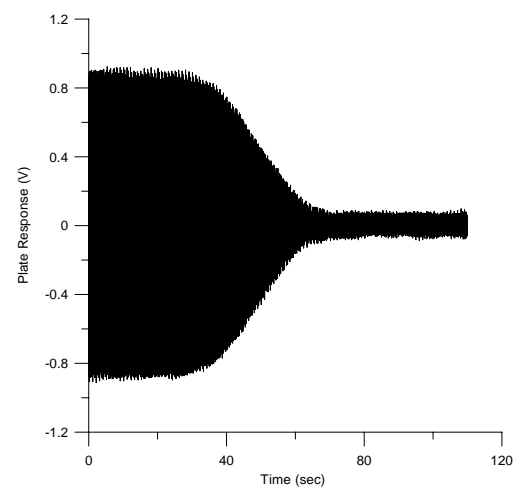

(a)

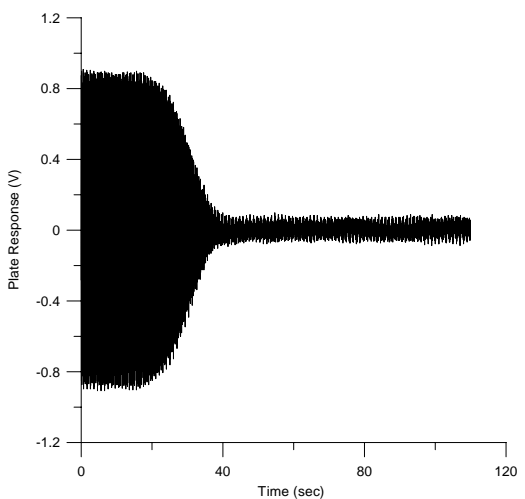

(c)

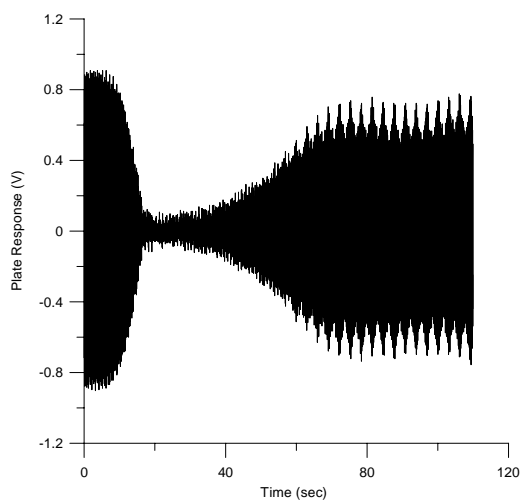

(e)

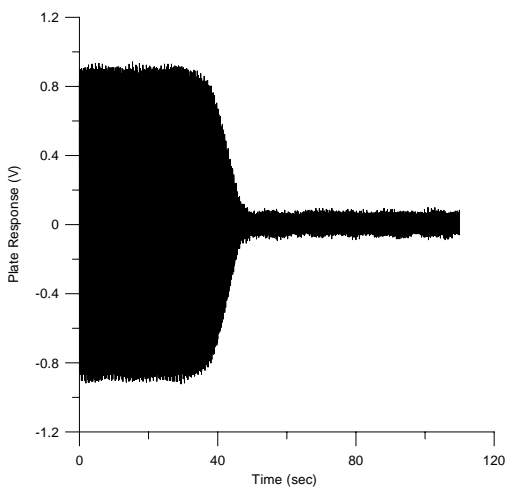

(b)

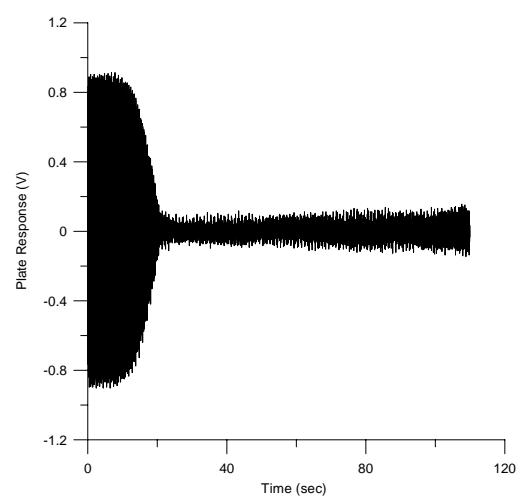

(d)

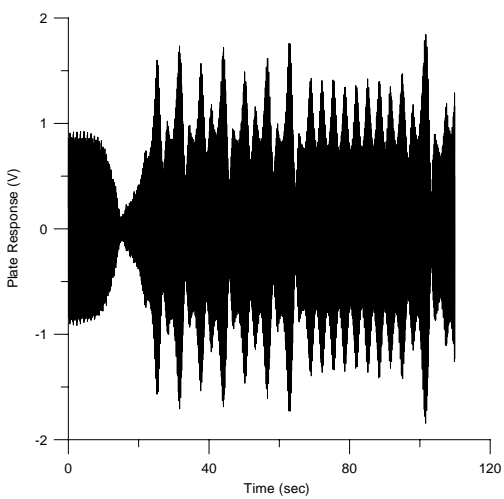

(f)

Figure 5.13: Effect of varying the feedback gain $\alpha$ : (a) $\alpha=0.04$, (b) $\alpha=0.07$, (c) $\alpha=0.1$, (d) $\alpha=0.11$, (e) $\alpha=0.12$, and (f) $\alpha=0.15$. 
steady state and the rate of decay are functions of the initial displacement, as shown in Fig. 5.14. The transient time is reduced from around $60 \mathrm{sec}$ for the initial displacement $0.03 \mathrm{~V}$ to around $30 \mathrm{sec}$ for the initial displacement $3.0 \mathrm{~V}$. Also, the rate of decay for the $0.03 \mathrm{~V}$ case is more gradual than in the first case. For larger values, the transient time is greater.

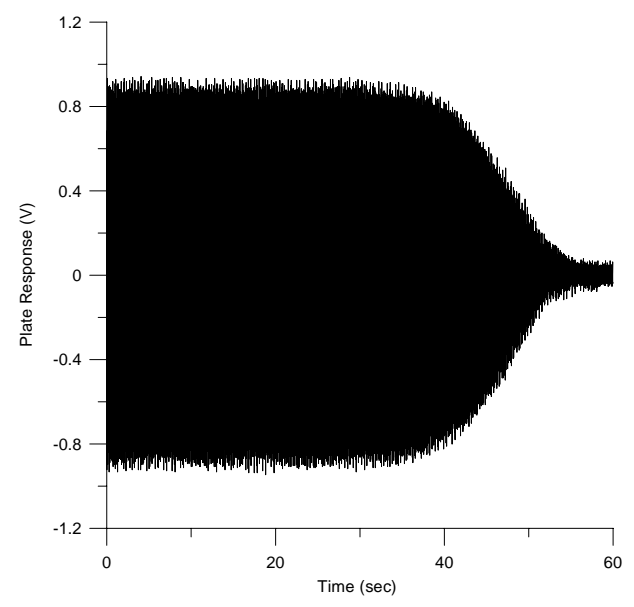

(a)

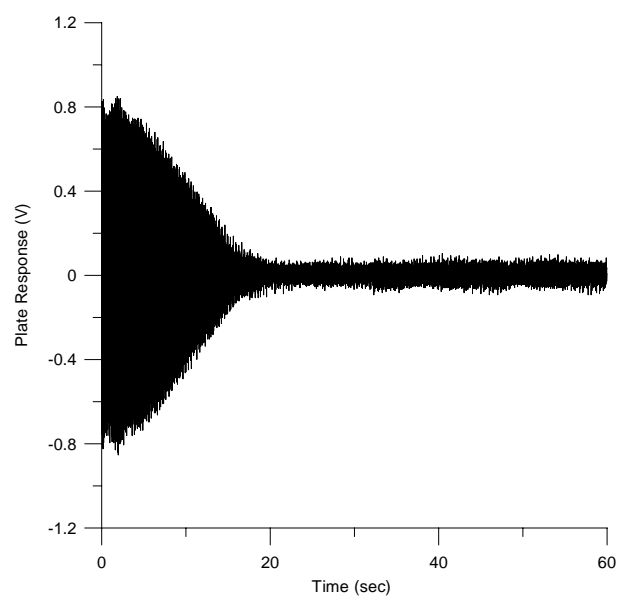

(b)

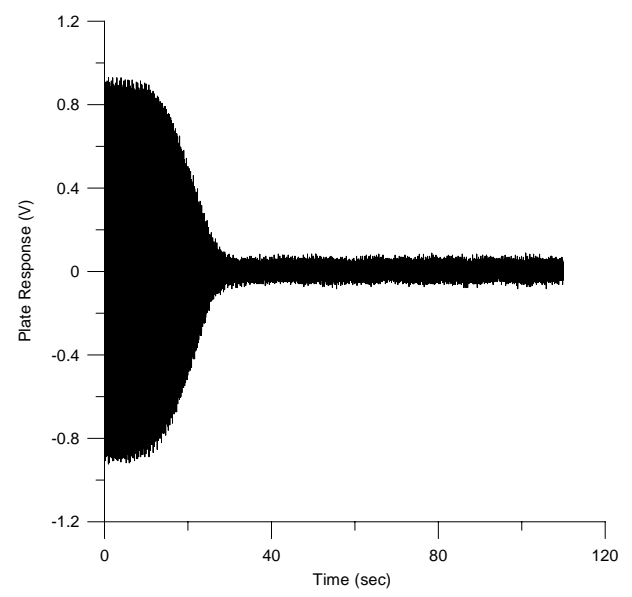

(c)

Figure 5.14: Effect of varying the initial displacement of the controller: (a) $v=0.03 \mathrm{~V}$, (b) $v=3.0 \mathrm{~V}$, and (c) $v=5.0 \mathrm{~V}$. 
Finally, frequency-response and force-response curves are shown for the second-mode control. In Fig. 5.15, frequency-response curves are shown for both the open-loop and closed-loop responses. The open-loop response follows the familiar anti-resonance behavior. However, the closed loop response is constant at a negligible value over a wide range of frequencies around the resonance frequency $\left(f_{n}=36.8 \mathrm{~Hz}\right)$. In Fig. 5.16, force-response curves are shown. Again, the open-loop response is almost linear as expected. The closed-loop response saturates beyond $F \approx 46 \mathrm{mg}$.

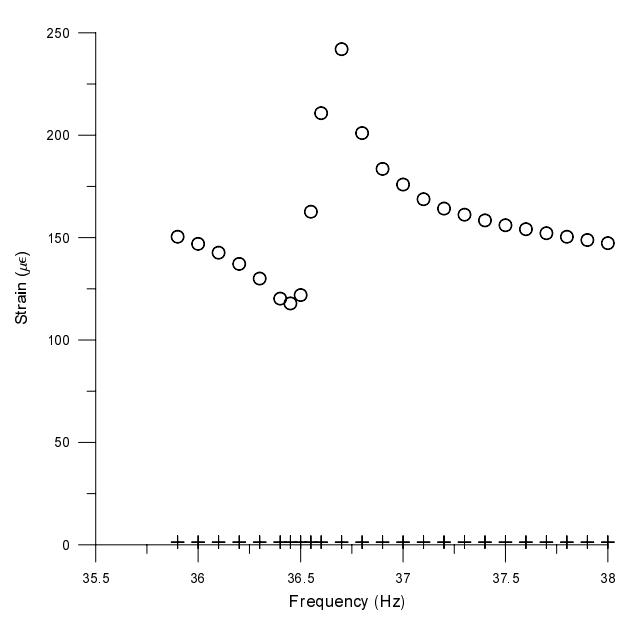

Figure 5.15: Frequency-response curves for the second mode: (ooo) controller off and $(+++)$ controller on.

\subsubsection{Simultaneous Control of the First Two Modes}

In this set of experiments, we simultaneously excite the first two modes of the plate: the first bending mode and the first torsional mode. The excitation level is $5.2 \mathrm{mg}$ for the first mode; while for the second mode, the excitation level is $958 \mathrm{mg}$. The natural frequencies are identified at $f_{n}=17.9 \mathrm{~Hz}$ for the first mode and $f_{n}=36.8 \mathrm{~Hz}$ for the second mode. Here 


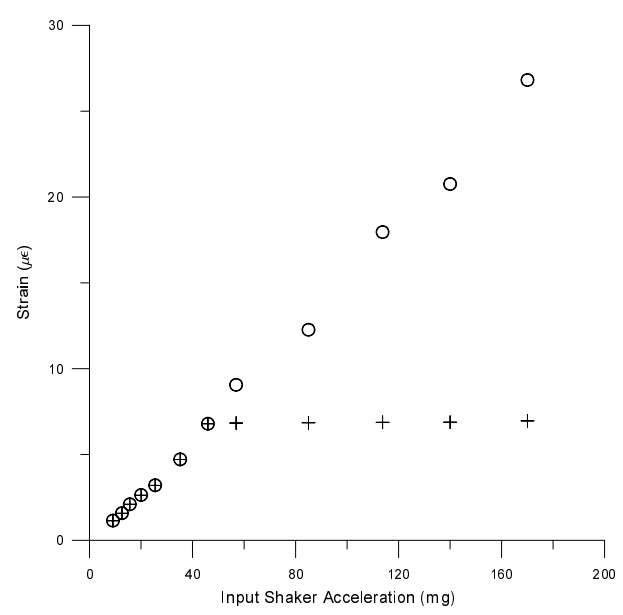

Figure 5.16: Force-response curves for the second mode: (ooo) controller off and $(+++)$ controller on.

we use two controllers, one for each mode. For the actuators and sensors, a pair of PZT actuators per mode and a single strain gauge is used. Figure 5.17(a) shows the response of the plate when activating the two controllers, while Fig. 5.17(b) shows the output of the two controllers together. The feedback gain $\alpha$ is 0.015 and the control gain $\gamma$ is 1 . As clear from the figure, our control strategy is as effective for two-mode control as it is for single-mode control. Each controller is coupled to the mode that is internally resonant with it. The output of the two controllers shows that the steady-state response is attained at $t \approx 100 \mathrm{sec}$. About a $30 \mathrm{~dB}$ reduction in the amplitude is noted for this case.

Next, we investigate the effect of changing the feedback gain $\alpha$ on the response as we did before. Figures 5.18(a), (b), and (c) show the plate response for the values of $\alpha$ of 0.01 , 0.02 , and 0.05 , respectively. Here, we note the same trend as for the single-mode control; namely, the steady-state response is attained earlier for higher values of $\alpha$ and the steadystate amplitude decreases with increasing values of $\alpha$. Again, this validates the perturbation 


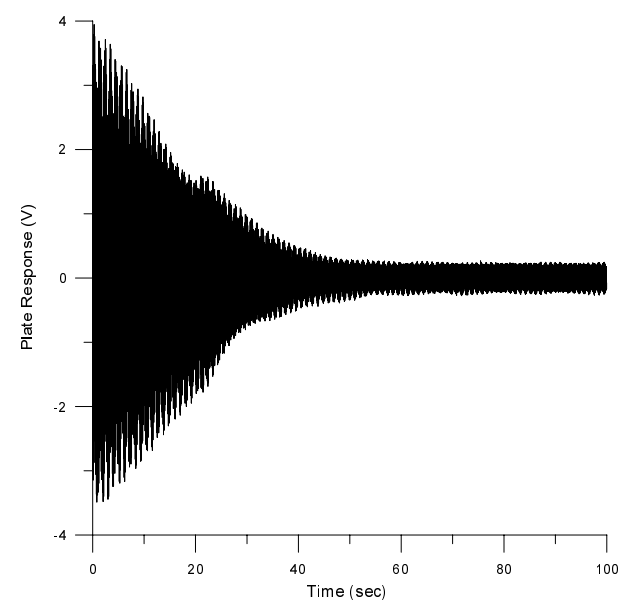

(a)

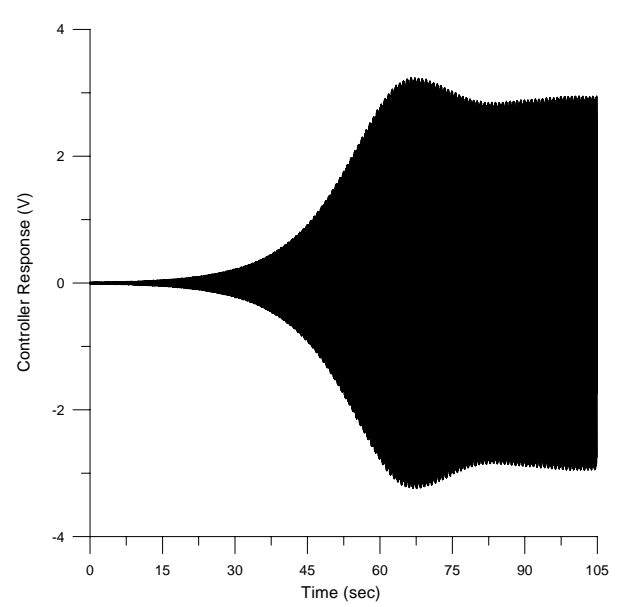

(b)

Figure 5.17: Simultaneous control of the first two modes of the plate: (a) plate response and (b) response of the two controllers.

solution presented in Chapter 2. In Fig. 5.19(a), we show the FFT results for the plate with no control, while the FFT results for the plate response after control is shown in Fig. $5.19(\mathrm{~b})$.

To further examine the robustness of the control strategy, we consider the case in which a noise signal is present. Here, we add a third harmonic signal at a frequency of $f=18.9 \mathrm{~Hz}$. The objective is to validate the filtering process, by which each controller is only coupled with the mode that is internally resonant with it. Since no controller is added to suppress this noise signal, we expect the response due to this signal to stay unchanged after activating the controllers. In Fig. 5.20(a), we show the power spectrum of the plate response before control. The power spectrum of the plate response after control is shown in Fig. 5.20(b). The activation of the controllers results in about a $45 \mathrm{~dB}$ reduction for the two modes they are 


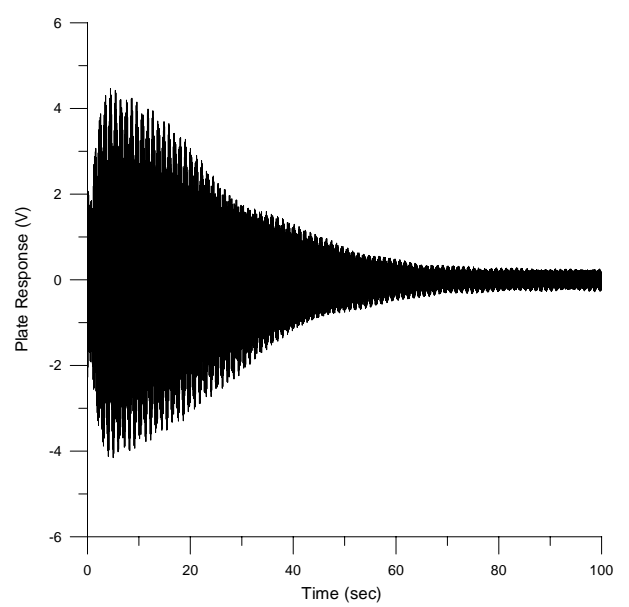

(a)

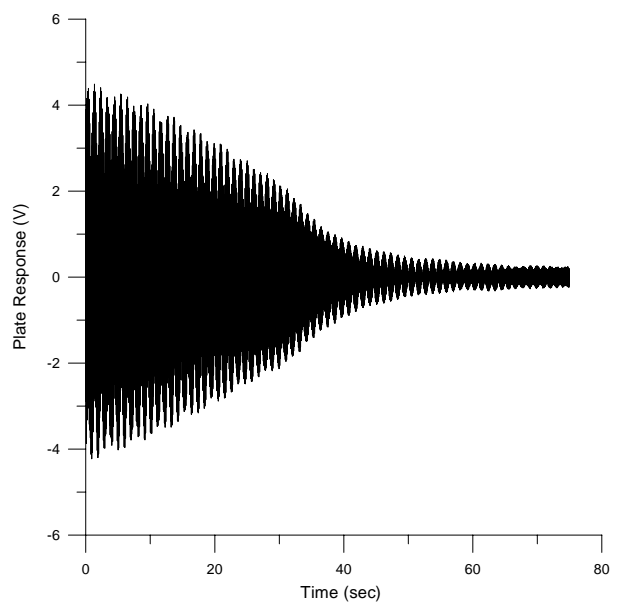

(b)

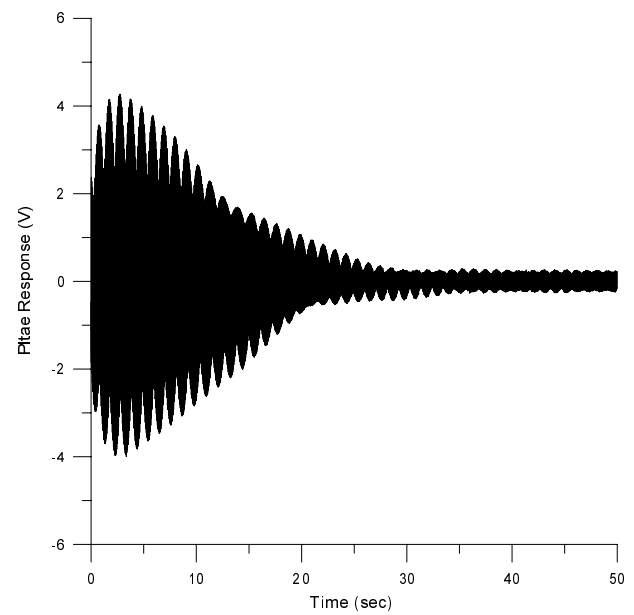

(c)

Figure 5.18: Effect of varying the feedback gain $\alpha$ : (a) $\alpha=0.01$, (b) $\alpha=0.02$, and (c) $\alpha=0.05$.

coupled with. However, the response due to the noise signal remains unchanged at around $-20 \mathrm{~dB}$. This clearly validates the filtering process and stresses the need for a controller for each excited mode. 


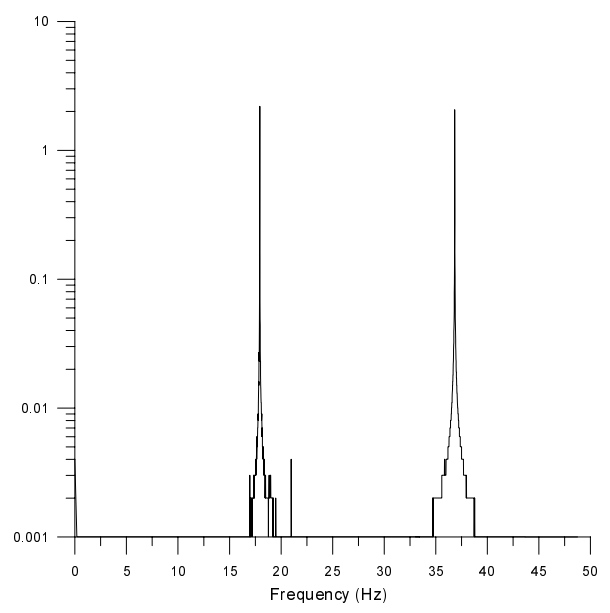

(a)

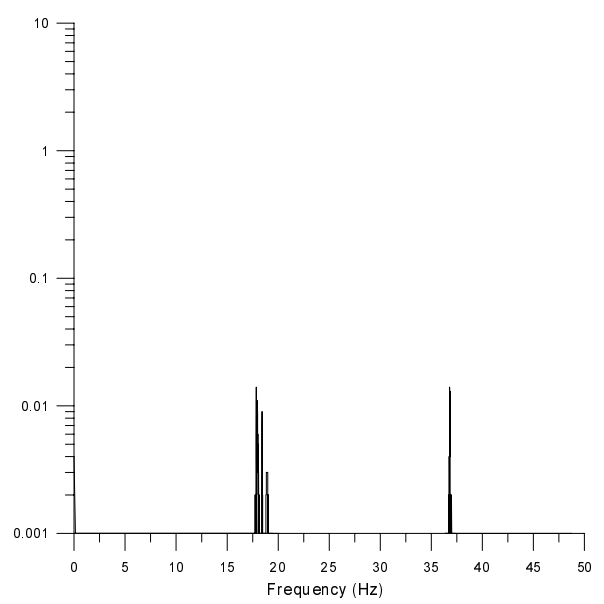

(b)

Figure 5.19: FFT results for the plate response: (a) no control and (b) with control.

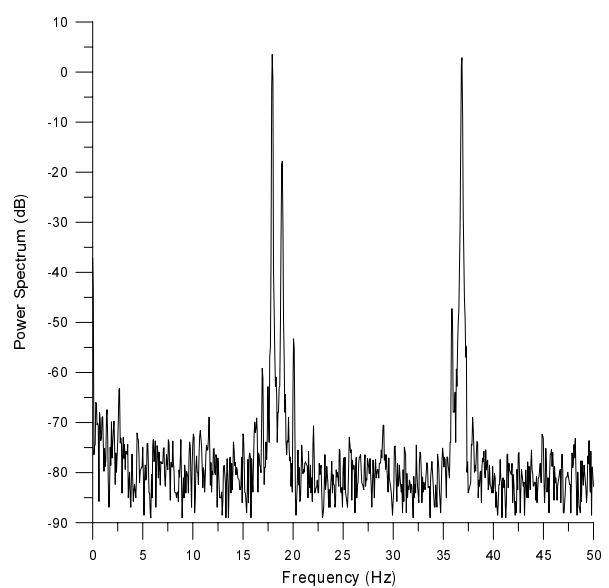

(a)

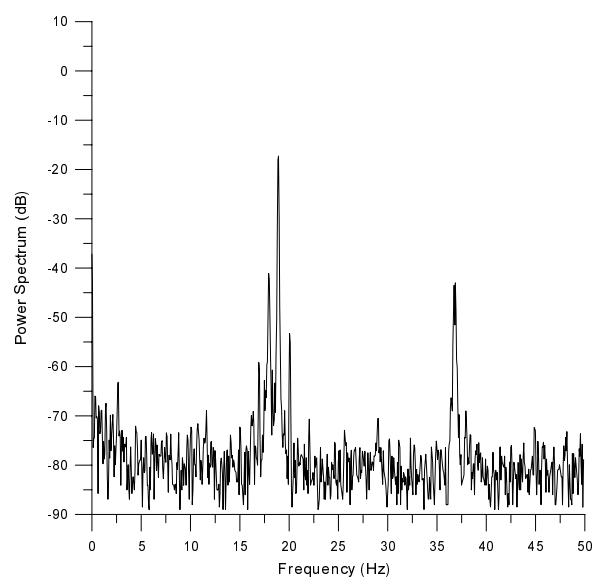

(b)

Figure 5.20: Power spectrum for the plate response with a noise signal: (a) no control and (b) with control. 


\section{Chapter 6}

\section{Adaptive Control}

Adaptive control makes it possible for the system (or structure) to adapt to a variable environment in the interest of continuously optimizing its performance. For our control strategy, tuning the controller frequency to one-half that of the system and maintaining this tuned frequency in the presence of external disturbances is desirable. As we mentioned earlier, in nonlinear problems, the frequency of the system changes with the amplitude. So, a continuous tuning mechanism is needed to keep the controller's frequency tuned as the amplitude of the vibrating structure changes. Furthermore, any real-life control strategy should be able to deal with varying external conditions, which could change the system frequency. Also, the control strategy should be able to suppress the vibrations due to unpredictable inputs with unknown frequencies. Hence, we seek to efficiently enhance our control strategy to make it an adaptive one. Our goal is to maintain the two-to-one internal-resonance condition by automatically measuring the system frequency and feeding it back to the controller. 


\subsection{Frequency-Measurement Technique}

As we discussed earlier in the introduction and in the analysis of the system's stability, mistuning between the system and the controller frequencies could result in harmonic instabilities and even chaotic responses. Hence, our frequency-measurement mechanism should provide a high level of accuracy. Furthermore, the measuring mechanism should not be susceptible to noise. It should be able to work at a wide range of system frequencies.

To measure the system's frequency, we propose to use a two-input NAND gate with Schmitttrigger action on both inputs. The gate switches at different points for positive- and negativegoing signals. This integrated chip (IC) is used to convert real-time analog signals into digital TTL- (transistor-transistor-logic) or CMOS- (complementary-metal-oxide-silicon) level square waves. This IC has a high noise immunity and requires only one positive DC supply voltage. The output of a NAND logic gate will be in the low level as long as both inputs are in the high level. If one or both inputs snap to the low level, then the output will switch to the high level. In our case, one of the inputs is connected to the power supply and, hence, is always in the high position. The output of the gate will stay in the high level until the voltage on the other input crosses the Schmitt-trigger threshold voltage, which is referred to as the positive voltage $\left(V_{P}\right)$. In this case, the output of the gate snaps to the low level. It stays in this low level until the input voltage crosses the negative-going Schmitt-trigger threshold voltage, which is referred to as the negative voltage $\left(V_{N}\right)$. The difference between $V_{P}$ and $V_{N}$ is referred to as the hysteresis voltage $\left(V_{\text {hys }}\right)$ and is typically around $0.9 \mathrm{~V}$ for this IC. Clearly, for our application, we need a low hysteresis value to be able to measure the 
frequency of low-amplitude signals. Figure 6.1 shows the characteristics of NAND Schmitttrigger gates and the definitions of $V_{P}, V_{N}$, and $V_{\text {hys }}$. In the figure, $V_{H}$ and $V_{L}$ refer to the high and low output levels, respectively, which also correspond to the DC power supply voltage and ground levels. The input analog signal has to fall between $V_{H}$ and $V_{L}$. For the IC used in this work, $V_{H}$ can be as high as $20 \mathrm{~V}$.

Using this method, we were able to avoid using an ADC (analog to digital converter) card, in which case, small signal-to-noise ratios degrade the accuracy of the measurement technique. Hence, no fine tuning or amplitude search algorithms are needed as was reported by Oueini and Nayfeh (2000). Furthermore, our method makes it possible to incorporate the measurement technique in the modeling software SIMULINK and the real-time digital signal processing software dSPACE. Once the sine-wave analog signal has been converted into a TTL/CMOS-level square wave, the frequency can be easily measured using a simple algorithm that measures the time between two consecutive high-to-low or low-to-high snaps. The accuracy of this measuring technique is very high $(5 \mathrm{mHz})$ and, using appropriate DC offset and gain, we can measure signals whose amplitudes are as low as $1 \mathrm{mV}$.

\subsection{Adaptive Control of a Beam with a Terfenol-D Ac- tuator}

To test our frequency-measurement technique, we propose to use a Terfenol-D actuator to control the vibrations of a flexible beam. In addition, this will further examine the use of our active-control strategy with actuators other than piezoelectric transducers. Terfenol-D 


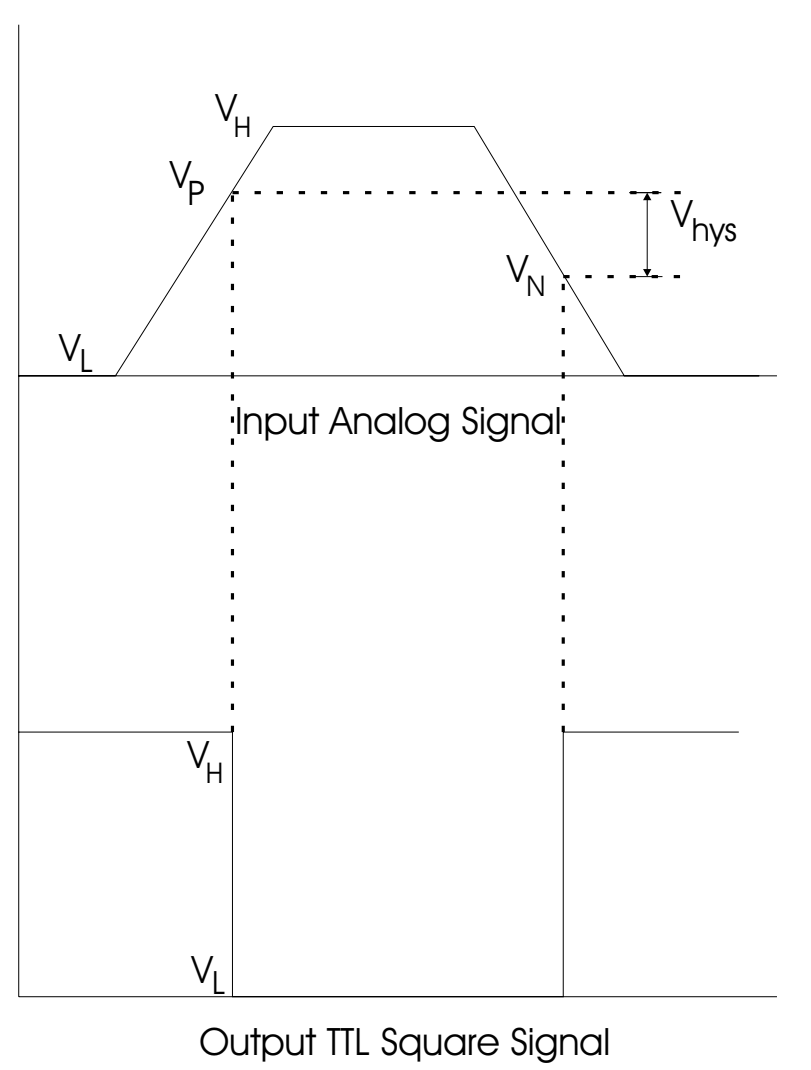

Figure 6.1: Characteristics of a NAND Schmitt-trigger gate.

is a magnetostrictive metal alloy that has a nonlinear constitutive relationship between the magnetic field and the strain. Unlike other reported applications, the Terfenol-D actuator used in this experiment is not biased. In other words, no permanent magnet is incorporated into the assembly of the actuator such that the small linear range is utilized. In the absence of a permanent magnet, it can be shown that the nonlinear strain-field relationship is predominantly quadratic. Hence, the saturation phenomenon can be utilized without the need for the controller to introduce the quadratic coupling.

Terfenol-D actuators are typically used for accurate positioning of mechanical loads and motion control industries. They are known to attain the highest displacement of any solid 
state actuator (Etrema Products, Inc.). They provide a high force with a microsecond response time. They function over a wide range of temperatures with a low-voltage operation. Terfenol-D alloys expand when placed in a magnetic field aligned with its magnetostrictive axis. This expansion is positive in either positive or negative magnetic field directions. Figure 6.2 shows a typical Terfenol-D actuator assembly. The generated magnetic field is proportional to the input current, which can be DC (for static applications) or AC (for dynamic applications). The used actuator is rated at a current of $3.2 \mathrm{~A} \mathrm{rms}$ and a temperature of $100^{\circ} \mathrm{C}$. Since no magnetic bias is introduced, the actuator does not operate at the frequency of the input current.

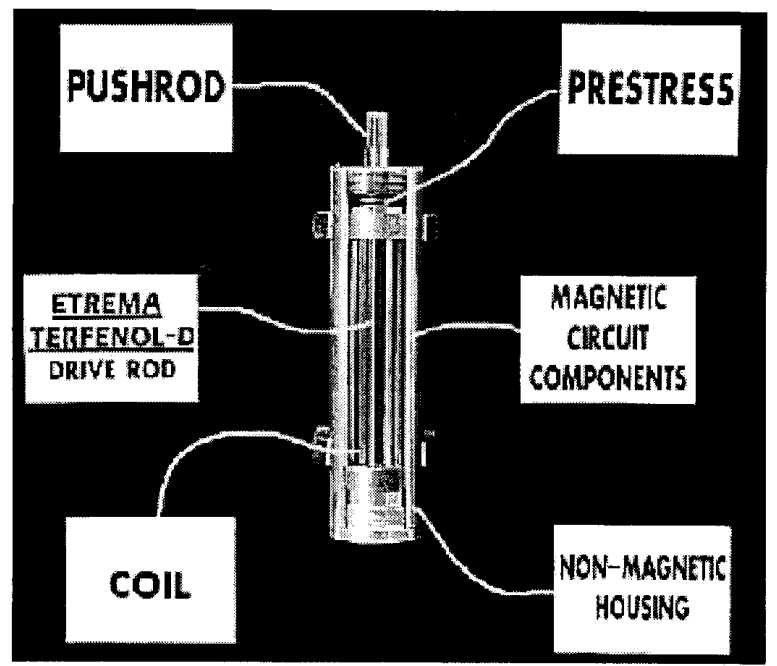

Figure 6.2: A typical assembly of a Terfenol-D actuator.

Figure 6.3 shows the displacement-current relationship for the used actuator as provided by the manufacturer. As clear from this figure, this relationship is predominantly quadratic. It can now be appreciated that unbiasing this actuator makes it operable over a much wider range of applied magnetic fields. The figure also shows that the effect of hysteresis should be 
included when modeling the mechanical behavior of this actuator. A model was developed by Pratt, Oueini, and Nayfeh (1999), which neither accounts for magnetic hysteresis nor includes eddy current losses. Their model results in a quadratic force-current relationship.

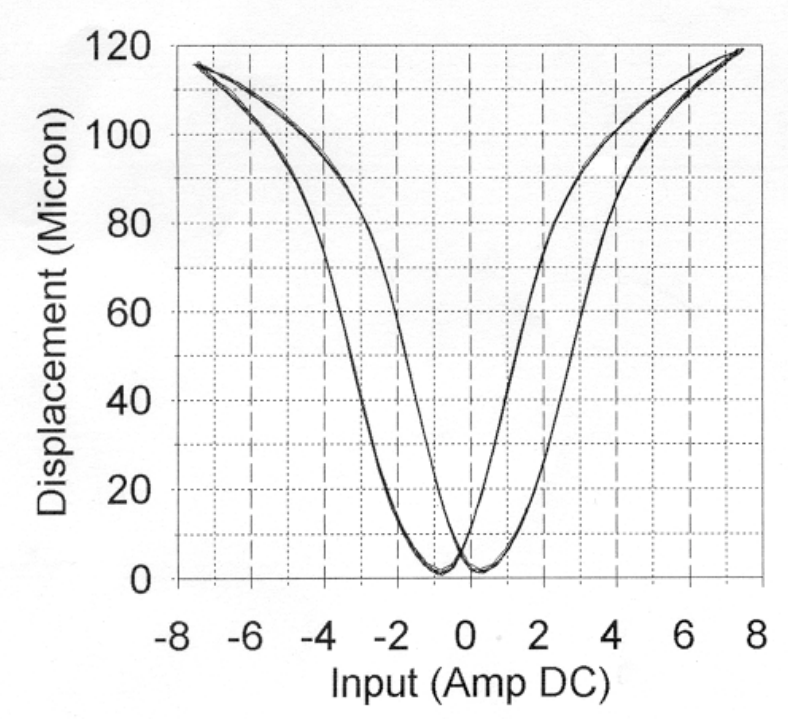

Figure 6.3: Displacement-current relationship for the used Terfenol-D actuator.

\subsubsection{Experimental Setup}

In this work, we control the lateral vibrations of a cantilever steel beam using a Terfenol-D actuator. The beam is externally excited by an electromagnetic shaker attached to the beam by a stinger. The actuator is attached near the root of the cantilever beam. The control technique as well as the system configuration are shown in Fig. 6.4. The setup consists of a signal generator, the shaker and its driving power amplifier, and an accelerometer mounted at the tip of the beam. The signal generator is used to drive the shaker at the desired amplitude and frequency. The accelerometer signal is then fed to the controller. The output 
of the controller is amplified and sent to the actuator. The outputs of both the beam and the controller are first filtered and then monitored by an oscilloscope and a signal analyzer. The filter used is a Butterworth IIR low-pass filter. An A/D card and a PC are used to collect the data.

The beam measurements are $53.66 \mathrm{~cm} \times 5.08 \mathrm{~cm} \times 0.43 \mathrm{~cm}$. The shaker is attached at a distance of $18.57 \mathrm{~cm}$ from the root, while the actuator is attached at a distance of $3.02 \mathrm{~cm}$ from the root. A 50-lb shaker is used with an excitation level of $35.8 \mathrm{mg}$. The first natural frequency is found as $f_{n}=11.875 \mathrm{~Hz}$ using a crude FRF analysis together with a fine-tuned frequency sweep.

The control technique is similar to the one used for the PZT actuator. We use a digital control system, which utilizes the modeling software SIMULINK and the dSPACE DS1103 controller installed in a PC. First, we use the SIMULINK software to build the control block diagram. This SIMULINK model is then used to generate a C-code model by the dSAPCE Real-Time Workshop. The dSPACE Real-Time Interface then connects this code with the dSPACE hardware for simulation. The block diagram of the controller is also shown in Fig. 6.4. Adaptive control is achieved by continuously measuring the frequency of the beam using the frequency-measurement technique discussed earlier. The controller frequency is set at one-half that of the measured frequency to exploit the saturation phenomenon. The other difference from the PZT-actuated case is that the output of the controller is not squared in this experiment. Instead, we let the actuator do the squaring because its behavior is predominantly quadratic. 


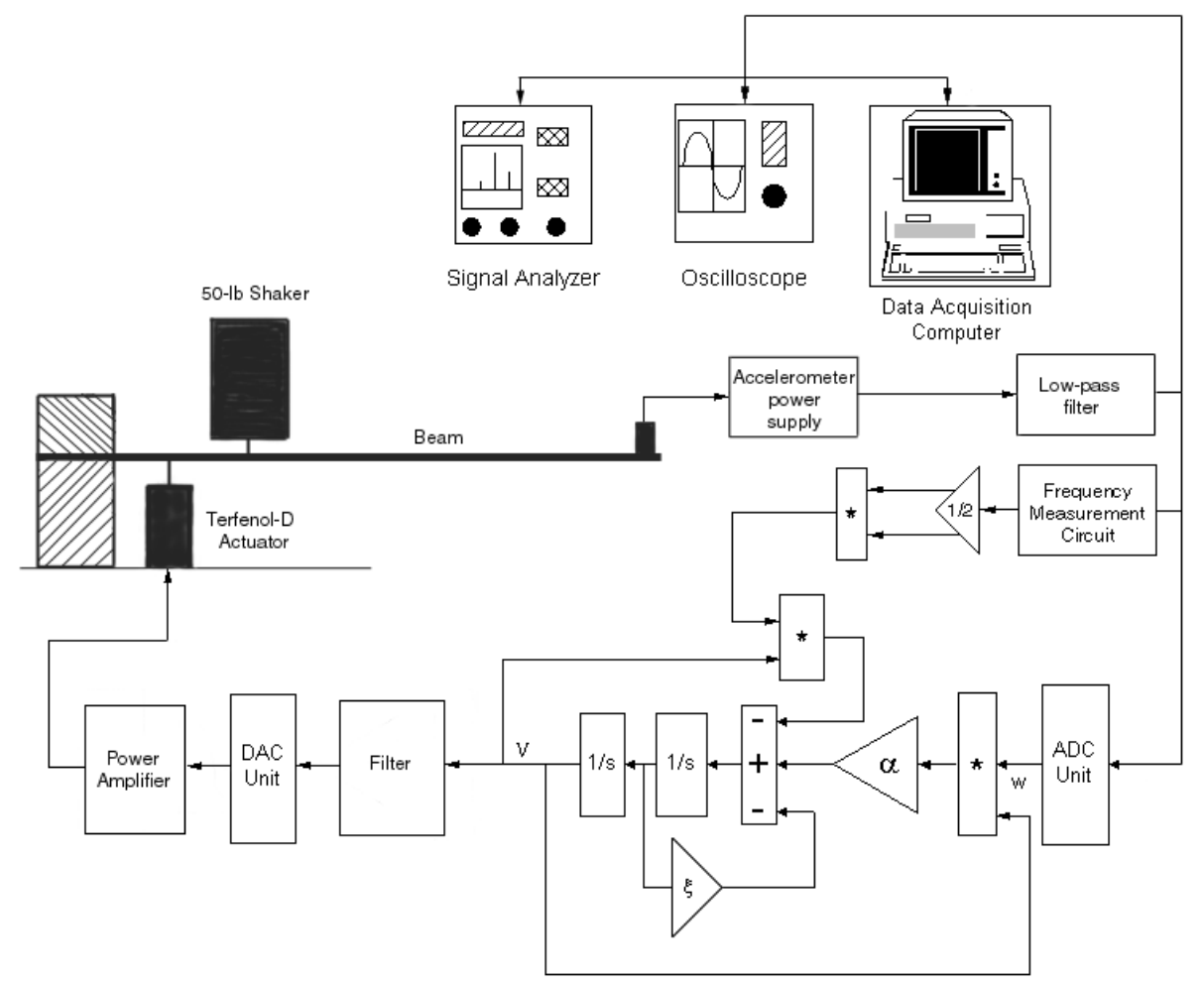

Figure 6.4: Experimental setup and control technique.

\subsubsection{Results}

We first conduct an experiment to verify the quadratic behavior of the actuator. To this end, we drive the actuator at a frequency of $13.31 \mathrm{~Hz}$. The beam length in this case is $34.61 \mathrm{~cm}$. The first natural frequency of the beam for this case is $f_{n}=26.62 \mathrm{~Hz}$. Hence, the actuator's driving frequency is one-half the natural frequency of the beam. Figure 6.5 shows the power spectrum of the output of the accelerometer. A superharmonic resonance of order 2 is observed, which indicates a quadratic nonlinearity. The even harmonics are clearly more dominant than the odd ones, which also indicates a predominant quadratic behavior.

Next, we drive the shaker at the natural frequency of the beam and employ the controller. 


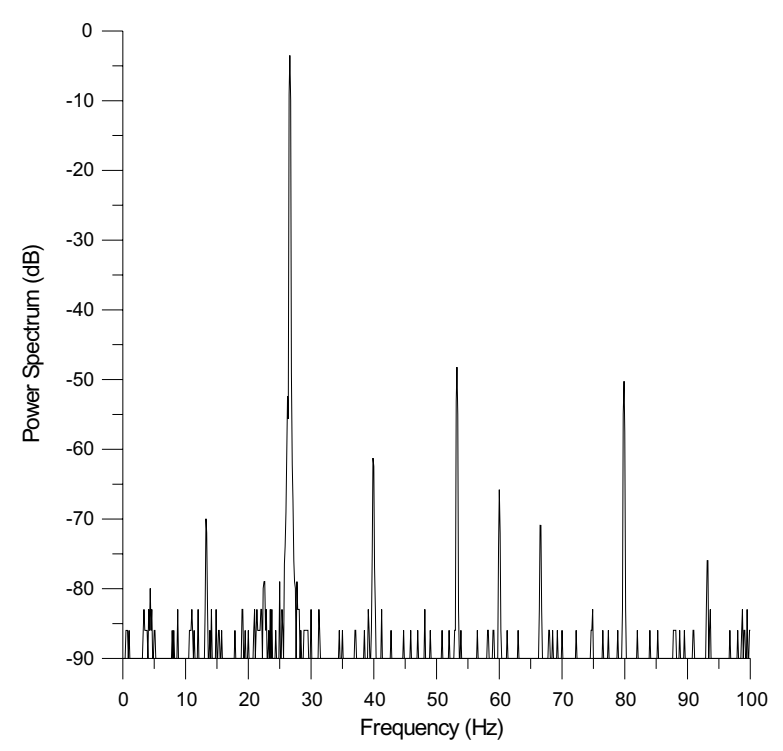

Figure 6.5: Power spectrum of the output of the beam when excited at $f=13.31 \mathrm{~Hz}$.

The output of the controller is fed to the actuator. The result is shown in Fig. 6.6 for a feedback gain $\alpha=0.05$ and a control gain $\gamma=3$. The controller is switched on simultaneously with the shaker. The output is collected for a long time $(120 \mathrm{sec})$ to make sure that steadystate response is attained. Figure 6.6(a) shows the beam response, while Fig. 6.6(b) shows the controller output. Figure 6.6(a) shows a great reduction in the beam response. About a $30 \mathrm{~dB}$ reduction is obtained for this case. The steady-state response is attained at $t \approx 60$ sec. The controller response (Fig. 6.6(b)) also exhibits the saturation phenomenon. This result is further validated by computing the FFT of the output of the beam before and after control. The result is shown in Fig. 6.7, where Fig. 6.7(a) shows the result with control and Fig. 6.7(b) shows the result without control. The second small peak in Fig. 6.7(a) is due to the actuator superharmonic resonance of order 4 .

Pratt, Oueini, and Nayfeh (1999) attempted to conduct the same experiment. However, their 


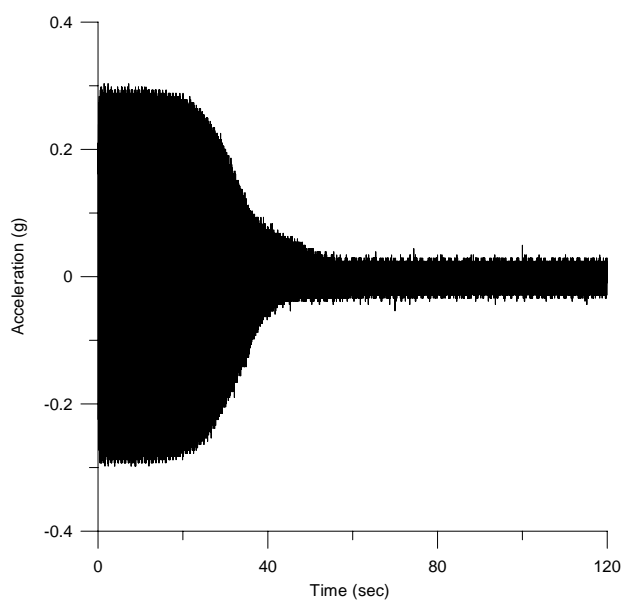

(a)

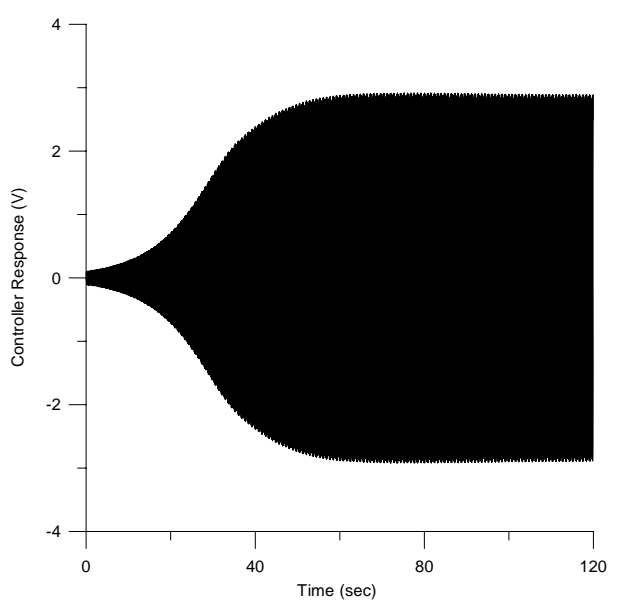

(b)

Figure 6.6: Control of the first mode of the beam when $f_{n}=11.875 \mathrm{~Hz}$ : (a) acceleration at the tip of the beam and (b) controller response.

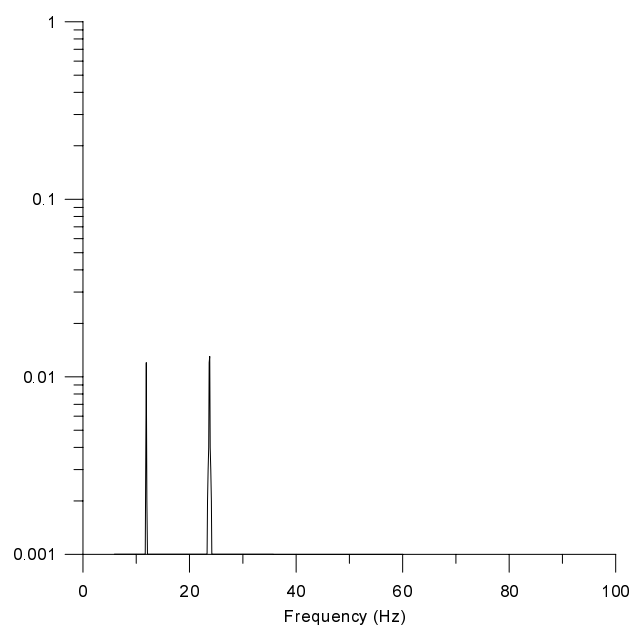

(a)

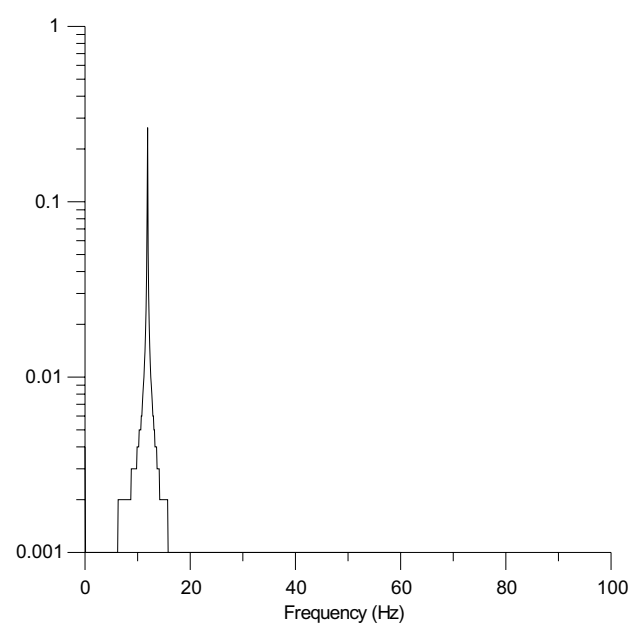

(b)

Figure 6.7: FFT of the beam acceleration at the tip: (a) with control and (b) without control. 
results showed that the system did not reach steady state. The controller response continued to grow with time and, hence, the saturation phenomenon was not exhibited. They suspected that the saturated response was unstable via a Hopf bifurcation. As we discussed in Chapter 2 and was also demonstrated by more than one researcher (for example, Balachandran and Nayfeh, 1991), a Hopf bifurcation results when mistuning occurs between the frequencies of the controller and the plant. To validate the assumption that mistuning was responsible for the anomaly in the experiments of Pratt, Oueini, and Nayfeh, we repeat the experiment without the frequency-measurement mechanism. Here, we fix the controller frequency at one-half of $11.875 \mathrm{~Hz}$ and bypass the frequency-measurement block. The result is shown in Fig. 6.8 for the beam acceleration at the tip $(6.8(\mathrm{a}))$ and the controller response $(6.8(\mathrm{~b}))$. As clear from the figure, saturation is not attained in this case. The controller response continues to grow, and the beam response goes in and out of tune. This result clearly shows the significance of adding the frequency-measurement mechanism. This is especially the case in nonlinear problems, where the frequency of oscillations depends on the amplitude. 


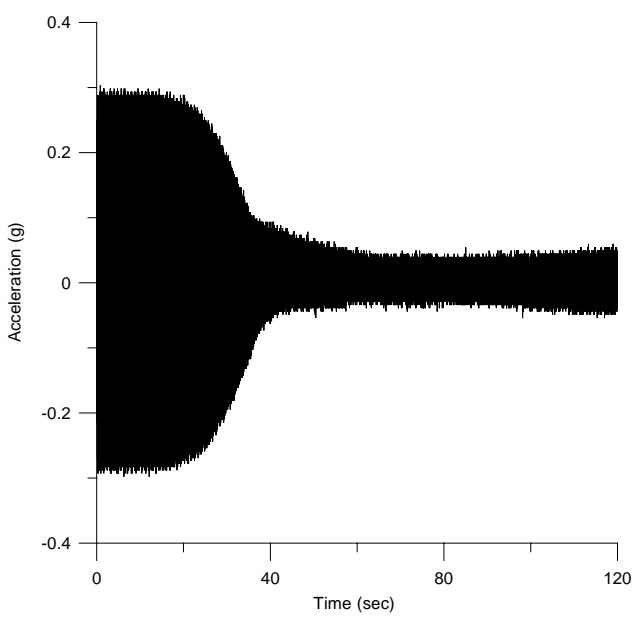

(a)

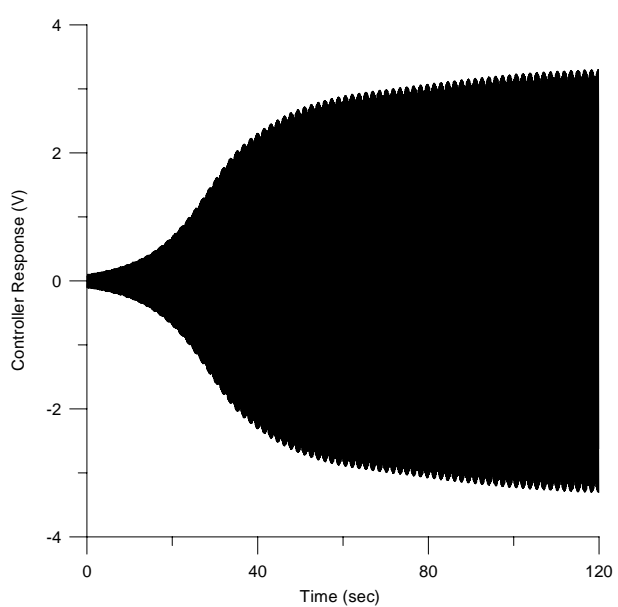

(b)

Figure 6.8: Nonadaptive control of the first mode of the beam: (a) acceleration at the tip of the beam and (b) controller response. 


\section{Chapter 7}

\section{Concluding Remarks and Recommendations}

\section{$7.1 \quad$ Summary}

We consider experimental and numerical nonlinear control of two-dimensional systems. As a case study, we consider the lateral vibrations of plates. In this regard, the saturation phenomenon is utilized to suppress the high-amplitude bending and torsional vibrational modes of rectangular cantilever plates. A second-order electronic circuit (controller) is coupled to the plant through appropriate sensors and actuators. Furthermore, a two-to-one internal-resonance condition is maintained between the plant and the controller. In this case, energy is transferred completely from one part of the combined system to the other. When the plant is forced at resonance, this energy-transfer mechanism limits the response of the plant. Both experimental and numerical results are obtained and compared to each other. This comparison is excellent. Also, an efficient frequency-tuning mechanism such that the two-to-one internal resonance condition is maintained is developed. Hence, the control 
strategy is made adaptive and robust. It can now be applied to nonlinear problems, where the frequency of the system changes as its amplitude changes. In addition, an optimization study is conducted to locate the optimum location of the actuators in order to maximize the control effort.

\subsubsection{Analytical Considerations}

We derive the equation of motion for a cantilever rectangular plate excited by a shaker and acted upon by an actuator using the extended Hamilton principle. As an actuator, we use a pair of PZT ceramics. An expression for the external bending moments induced by the actuator is derived for the case of perfect bonding. The equation of motion for the vibrating plate is solved analytically using perturbation methods for the case of external forcing near resonance. In this regard, the method of multiple scales is applied to the partial-differential equation of motion and associated boundary conditions. The analytical solution for the response of the plate shows the amplitude to be independent of the forcing amplitude above a critical value. This is in contrast with the linear solution without control, where the amplitude of oscillations is directly proportional to the forcing amplitude. The saturated response is only a function of the controller damping coefficient and the feedback gain. On the other hand, the controller response is a function of the nonlinear coupling gains, the damping coefficients for the plate and the controller, the detuning parameter, and the forcing amplitude. Hence, as we increase the forcing amplitude, the added energy is channeled to the controller, while the plant response stays unchanged. The saturated response is stable as long as the exciting frequency is tuned to twice the controller frequency. 


\subsubsection{Numerical Results}

We present two numerical methods to solve for the plate and the controller responses. In the first one, the plate, the actuator, and the controller are modeled using a finite-element package and the mathematical model is fully integrated over time. The finite-element package is modified to allow for feedback control using the saturation phenomenon. In the second method, the natural modes and frequencies of the plate are obtained using the finite-element package. These modes are then used in modal analysis to solve for the plate and controller responses. The numerical results obtained by the first method agree very well with the analytical perturbation solution and with the experimental results. The frequency-response curves obtained by the second method reveal the existence of a second resonance peak away from the original uncontrolled resonance. This new resonance results from the autoparametric coupling between the controller and the plant.

\subsubsection{Optimal Placement of Actuators}

We conduct a numerical study to locate the optimum actuator locations on the structure to be controlled. Our objective is to minimize the control effort and maximize the controllability of the system. To this end, we present two methods. The first is based on maximizing the control gain factor, which was derived during the analytical study. This method is specific to the case in which the actuator extends over a finite area on the structure, such as the case of a PZT actuator. The second method is more general and is based on a global measure of controllability for first-order linear systems. Both methods give the locations of maximum 
controllability measures for a certain mode of vibration.

\subsubsection{Experimental Work}

We design an experimental setup made of sensors, actuators, and electronic devices to verify the effectiveness of the control strategy. A strain gage is used as a sensor, while a PZT ceramic is used as an actuator. As a controller, we use a digital control system, which utilizes the modeling software SIMULINK and the dSPACE controller board. The controller frequency is set at one-half that of the plate to exploit the saturation phenomenon.

First, we consider suppressing the first bending mode of the plate. The control strategy is found to be very efficient in virtually eliminating the high-amplitude vibrations caused by the shaker. A parametric study is also conducted to relate the effect of changing the nonlinear gains, controller damping coefficient, and initial conditions. Frequency-response curves are constructed to show that the controller is broadband, while force-response curves are generated to verify the saturation phenomenon. The results of the parametric study confirm the analytical perturbation solution for both the plate and the controller responses. Furthermore, the experimentally obtained frequency-response curves verify the broadband effectiveness of the control strategy. Also, the amplitude of the plate saturates at a constant value after a critical forcing level, in agreement with the analytical solution. A similar set of experiments is also conducted for controlling the first torsional mode of the plate. Again, the control strategy is very effective in suppressing this mode, and the parametric study confirms the perturbation solution. 
In the third set of experiments, the first two modes of the plate are suppressed simultaneously. In this regard, two controllers are used, one for each mode. In this case, the control strategy is as effective as it is for single-mode control. The results also confirm the filtering process of the control strategy, in which each controller is only coupled with the mode that is internally resonant with it.

\subsubsection{Adaptive Control}

We tackle the problem of maintaining the two-to-one internal-resonance condition by continuously tuning the controller frequency to one-half that of the plate. Hence, the control strategy is made adaptive. This is especially crucial in nonlinear problems, where the frequency of the system changes with the amplitude. In this work, we maintain the internal-resonance condition by automatically measuring the system frequency and feeding it back to the controller. The frequency-measurement mechanism consists of a logic gate with Schmitt-trigger action on the input. This mechanism is very accurate and immune to noise disturbances. To test this mechanism, we use it in a problem for which nonadaptive control does not work. Here, we use a Terfenol-D actuator to control the vibrations of a flexible beam. This actuator is known to have a nonlinear strain-field relationship, with a predominant quadratic behavior. The results show that the frequency-measurement technique is very efficient. The response of the beam is greatly reduced and saturated at a small value. 


\subsection{Recommendations for Future Work}

The work presented in this Dissertation can be expanded to include the following tasks:

- The proposed control strategy needs to be applied to other two-dimensional systems, such as shells. Also, it needs to be tested on real-life structures, such as airplane wings, helicopter blades, automotive applications, manufacturing processes, motion control, and measurement systems.

- The developed numerical method needs to be tested in simulating the controlled response of real-life complicated structures.

- The current work provides a proof of concept for applying the control strategy in nonlinear problems. More experimental work needs to be conducted to verify the applicability of this control strategy to nonlinear problems. In this regard, we propose applying it to systems with strong nonlinearities, such as square cantilever beams, which possess a one-to-one internal resonance. The frequency-measurement mechanism needs to be tested in such circumstances to test its robustness and reliability in stronglynonlinear systems.

- More analytical work needs to be conducted using perturbation methods to control systems with cubic and quadratic nonlinearities. Here, the method of multiple scales needs to be applied to a system of first-order equations, rather than the second-order original system. 
- The developed numerical actuator-location optimization techniques need to be tested experimentally. In this regard, a set of collocated actuators should be attached to the structure. The control strategy is then applied for each actuator using the same gain values. The results are then compared to locate the optimum location with the least control effort.

- A study needs to be conducted to optimize the size and number of actuators.

- A study needs to be conducted to optimize the location, size, and number of sensors on the structure. 


\section{Bibliography}

1. Agnes, G. and Inman, D., "Nonlinear piezoelectric vibration absorbers," Journal of Smart Materials and Structures, vol. 5, pp. 704-714, 1996.

2. Arnold, F., "Steady-state behavior of systems provided with nonlinear dynamic vibration absorbers," Journal of Applied Mechanics, vol. 22, pp. 487-492, 1955.

3. Ashworth, R. and Barr, A., "The resonances of structures with quadratic inertial nonlinearity under direct and parametric harmonic excitation," Journal of Sound and Vibration, vol. 118, pp. 47-68, 1987.

4. Bajaj, A., Chang, S., and Johnson, J., "Amplitude modulated dynamics of a resonantly excited autoparametric two degree-of-freedom system," Nonlinear Dynamics, vol. 5, pp. 433-457, 1994.

5. Balachandran, B. and Nayfeh, A., "Observations of modal interactions in resonantly forced beam-mass structures," Nonlinear Dynamics, vol. 2, pp. 77-117, 1991.

6. Banerjee, B., Bajaj, A., and Davies, P., "Second order averaging study of an autoparametric system," in Nonlinear Vibrations DE-Vol. 54 (Ibrahim, R., Bajaj, A., and Bergman, L., eds.), pp. 127-138, ASME, 1993.

7. Browning, D. and Wynn, W., "Multiple-mode piezoelectric passive damping experiments for an elastic plate," Proceedings of the 11th International Modal Analysis Conference, Kissimmee, Florida, pp. 1520-1526, 1993.

8. Burdisso, R. and Heilmann, J., "A new dual-reaction mass dynamic vibration absorber actuator for active vibration control," Journal of Sound and Vibration, vol. 214, no. 5, pp.817-831, 1998.

9. Cartmell, M. and Roberts, J., "Simultaneous combination resonances in an autoparametrically resonant system," Journal of Sound and Vibration, vol. 123, pp. 81-101, 1988.

10. Cartmell, M. and Lawson, J., "Performance enhancement of an autoparametric vibration absorber by means of computer control," Journal of Sound and Vibration, vol. 177, pp. 173-195, 1994. 
11. Crawley, E. and de Luis, J., "Use of piezoelectric actuators as elements of intelligent structures," AIAA Journal, vol. 25, no. 10, pp. 1373-1385, 1987.

12. Cuvlaci, O. and Ertas, A., "Pendulum as vibration absorber for flexible structures: Experiments and theory," Journal of Vibration and Acoustics, vol. 118, pp. 558-566, 1996.

13. Davis, C., Lesieutre, G., and Dosch, J., "A tunable electrically shunted piezoceramic vibration absorber," Proceedings of SPIE, The International Society for Optical Engineering, vol. 3045, pp. 51-59, 1997.

14. Den Hartog, J., Mechanical Vibrations, McGraw-Hill, New York, 1956.

15. Duquette, A. P., Tuer, K. L., and Golnaraghi, M. F., "Vibration control of a flexible beam using a rotational internal resonance controller Part II: Experiment," Journal of Sound and Vibration, vol. 167, no. 1, pp. 63-75, 1993.

16. Fanson, J. and Caughey, T., "Positive position feedback control for large space structures," AIAA Journal, vol. 28, no. 4, pp. 717-724, 1990.

17. Filipovic, D. and Schroder, D., "Vibration absorption with linear active resonators: Continuous and discrete time design and analysis," Journal of Vibration and Control, vol. 5, pp. 685-708, 1999.

18. Frahm, H., "Device for damping vibrations of bodies." US Patent No. 989958, 1909.

19. Francheck, M., Ryan, M., and Bernhard, R., "Adaptive passive vibration control," Journal of Sound and Vibration, vol. 189, no. 5, pp. 565-585, 1995.

20. Golnaraghi, M., "Regulation of flexible structures via nonlinear coupling," Dynamics and Control, vol. 1, pp. 405-428, 1991.

21. Gonsalves, D., Neilson, R., and Barr, A., "The dynamics and design of a non-linear vibration absorber," Journal of Mechanical Engineering Science, vol. 207, pp. 363-374, 1993.

22. Haddow, A., Barr, A., and Mook, D., "Theoretical and experimental study of modal interaction in a two-degree-of-freedom structure," Journal of Sound and Vibration, vol. 97, no. 3, pp. 451-473, 1984.

23. Hagood, N. and Crawley, E., "Experimental investigation into passive damping enhancement for space structures," Proceedings of the 30th AIAA/ASME/ASC/AHS Structures Structural Dynamics and Materials Conference, Mobile, Alabama, AIAA Paper 89-3436, pp. 97-109, 1989.

24. Hagood, N. and Von Flotow, A., "Damping of structural vibrations with piezoelectric materials and passive electrical networks," Journal of Sound and Vibration, vol. 146, pp. 243-268, 1991. 
25. Hamdan, A. M. A. and Nayfeh, A. H., "Measures of modal controllability and observability for first- and second-order linear systems," Journal of Guidance, Control, and Dynamics, vol. 12, no. 3, pp. 421-428, 1989.

26. Hatwal, H., Mallik, A., and Ghosh, A., "Non-linear vibrations of a harmonically excited autoparametric system," Journal of Sound and Vibration, vol. 81, no. 2, pp. 153-164, 1982.

27. Hatwal, H., Mallik, A., and Ghosh, A., "Forced nonlinear oscillation of an autoparametric system - Part 1: Periodic Responses," Journal of Applied Mechanics, vol. 50, pp. 657-662, 1983.

28. Haxton, R. and Barr, A., "The autoparametric vibration absorber," Journal of Engineering for Industry, vol. 94, pp. 119-125, 1972.

29. Hollkamp, J., "Multimodal passive vibration suppression with piezoelectric materials and resonant shunts," Journal of Intelligent Material Systems and Structures, vol. 5, no. 1, pp. 49-57, 1994.

30. Hollkamp, J. and Starchville, T., "A self-tuning piezoelectric vibration absorber," Journal of Intelligent Material Systems and Structures, vol. 5, pp. 559-566, 1994.

31. Hosek, M., Olgac, N., and Elmali, H., "The centrifugal delayed resonator as a tunable torsional vibration absorber for multi-degree-of-freedom systems," Journal of Vibration and Control, vol. 5, no. 2, pp. 299-322, 1999.

32. Huang, S. and Lian, R., "A dynamic absorber with active vibration control," Journal of Sound and Vibration, vol. 178, no. 3, pp.323-335, 1994.

33. Hunt, J. and Nissen, J., "The broadband dynamic absorber," Journal of Sound and Vibration, vol. 83, pp. 573-578, 1982.

34. Ibrahim, R. and Roberts, J., "Broad band random excitation of a two-degree-offreedom system with autoparametric coupling," Journal of Sound and Vibration, vol. 44, no. 3, pp. 335-348, 1976.

35. Kailath, T., Linear Systems. Prentice-Hall, Inc., Englewood Cliffs, New Jersey, 1980.

36. Khajepour, A. and Golnaraghi, M., "Experimental control of flexible structures using nonlinear modal coupling: Forced and free vibrations," Journal of Intelligent Material Systems and Structures, vol. 8, pp. 697-710, 1997.

37. Lee, C. and Shaw, S., "The non-linear dynamic response of paired centrifugal pendulum vibration absorbers," Journal of Sound and Vibration, vol. 203, no. 5, pp. 731-743, 1997.

38. Lesieutre, G., "Vibration damping and control using shunted piezoelectric materials," The Shock and Vibration Digest, vol. 30, no. 3, pp. 187-195, 1998. 
39. Margolis, D. and Baker, D., "The variable fulcrum isolator: a low power, nonlinear, vibration control component," Journal of Dynamic Systems, Measurement, and Control, vol. 114, pp. 148-154, 1992.

40. Meirovitch, L., Computational Methods in Structural Dynamics. Sijthoff \& Noordhoff, Maryland, 1980.

41. Meirovitch, L., Principles and Techniques of Vibrations. Prentice Hall, New Jersey, 1997.

42. Mianzo, L., "An adaptable vibration absorber to minimize steady state and start-up transient vibrations-an analytical and experimental study," M. Sc. Thesis, Pennsylvania State University, 1992.

43. Nayfeh, A., Mook, D., and Marshall, L., "Nonlinear coupling of pitch and roll modes in ship motion," Journal of Hydronautics, vol. 7, no. 4, pp. 145-152, 1973.

44. Nayfeh, A. and Mook, D., Nonlinear Oscillations. John Wiley \& Sons, Inc., New York, 1979.

45. Nayfeh, A., Introduction to Perturbation Techniques. John Wiley \& Sons, Inc., New York, 1981.

46. Nayfeh, A. and Zavodney, L., "Experimental observation of amplitude- and phasemodulated responses of two internally coupled oscillators to a harmonic excitation," Journal of Applied Mechanics, vol. 110, pp. 706-710, 1988.

47. Nayfeh, A. and Balachandran, B., Applied Nonlinear Dynamics. John Wiley \& Sons, Inc., New York, 1995.

48. Nayfeh, A., Nonlinear Interactions. John Wiley \& Sons, Inc., New York, 2000.

49. Nayfeh, T., Nayfeh, A., and Mook, D., "A theoretical and experimental investigation of a three-degree-of-freedom structure," Nonlinear Dynamics, vol. 6, pp. 353-374, 1994.

50. Oh, I., Nayfeh, A., and Mook, D., "A theoretical and experimental investigation of indirectly excited roll motion in ships," Philosophical Transactions of the Royal Society of London A, vol. 358, pp. 1853-1881, 2000.

51. Olgac, N. and Holm-Hansen, B., "A novel active vibration absorption technique: Delayed resonator," Journal of Sound and Vibration, vol. 176, pp. 93-104, 1994.

52. Olgac, N. and Jalili, N., "Modal analysis of flexible beams with delayed resonator vibration absorber: Theory and experiments," Journal of Sound and Vibration, vol. 218, no. 2, pp. 307-331, 1998.

53. Ormondroyd, J. and Den Hartog, J., "The theory of the dynamic vibration absorbers," Transactions of the ASME, vol. 50, PAPM-241, 1928. 
54. Oueini, S., Nayfeh, A., and Pratt, J., "A nonlinear vibration absorber for flexible structures," Nonlinear Dynamics, vol. 15, pp. 259-282, 1998.

55. Oueini, S. and Nayfeh, A., "Analysis and application of a nonlinear vibration absorber," Journal of Vibration and Control, vol. 6, no. 7, pp. 999-1016, 2000.

56. Oueini, S. and Golnaraghi, M. F., "Experimental implementation of the internal resonance control strategy," Journal of Sound and Vibration, vol. 191, no. 3, pp. 377-396, 1996.

57. Pai, P., Wen, B., Naser, A., and Schultz, M., "Structural vibration control using PZT patches and non-linear phenomena," Journal of Sound and Vibration, vol. 215, pp. 273-296, 1998.

58. Pratt, J., Oueini, S., and Nayfeh, A., "A Terfenol-D nonlinear vibration absorber," Journal of Intelligent Material Systems and Structures, vol. 10, pp. 29-35, 1999.

59. Puksand, H., "Optimum conditions for dynamic vibration absorbers for variable speed systems with rotating or reciprocating unbalance," The International Journal of Mechanical Engineering Education, vol. 3, no. 2, pp. 145-152, 1975.

60. Rice, H. and McCraith, J., "Practical non-linear vibration absorber design," Journal of Sound and Vibration, vol. 116, no. 3, pp. 545-559, 1987.

61. Roberson, R., "Synthesis of a nonlinear dynamic vibration absorber," Journal of the Franklin Institute, vol. 254, pp. 205-220, 1952.

62. Semercigil, S., Lammers, D., and Ying, Z., "A new tuned vibration absorber for wideband excitations," Journal of Sound and Vibration, vol. 156, pp. 445-459, 1992.

63. Shaw, J., Shaw, S., and Haddow, A., "On the response of the nonlinear vibration absorber," International Journal of Non-Linear Mechanics, vol. 24, pp. 281-293, 1989.

64. Snowdon, J., Vibration and Shock in Damped Mechanical Systems. John Wiley \& Sons, Inc., New York, 1968.

65. Soom, A. and Lee, M., "Optimal design of linear and nonlinear vibration absorbers for damped systems," Transactions of the ASME, vol. 105, pp. 112-119, 1983.

66. Timoshenko, S. and Woinowsky-Krieger, S., Theory of Plates and Shells. McGraw-Hill, 2nd edition, New York, 1959.

67. Von Flotow, A., Beard, H., and Bailey, D., "Adaptive tuned vibration absorbers: Tuning laws, tracking agility, sizing and physical implementations," Proceedings of Noise-Con 94: Progress in Noise Control for Industry, pp. 437-454, 1994. 
68. Waterman, E., "Vibration absorber with controllable resonance frequency." US Patent No. 4724923, 1988.

69. Watts, P., "On a method of reducing the rolling of ships at sea," Transactions of the Institution of Naval Architects, vol. 24, pp. 165-190, 1883.

70. Yabuno, H., Endo, Y., and Aoshima, N., "Stabilization of $\frac{1}{3}$-order subharmonic resonance using an autoparametric vibration absorber," Journal of Vibration and Acoustics, vol. 121, pp. 309-315, 1999. 


\section{Vita}

Osama Naim Ashour was born on June 14, 1968 in Kuwait City, Kuwait. He joined the Mechanical Engineering Department at Kuwait University in September 1986. He received his Bachelor of Science degree in June 1990. In August 1991, he joined the Department of Engineering Science and Mechanics at Virginia Polytechnic Institute and State University. He obtained his Master of Science degree in July 1993. Subsequently, he enrolled in the doctoral program and successfully defended his dissertation on January 17, 2001, to receive a Doctor of Philosophy degree in Engineering Mechanics. 